\title{
EQUICONTINUOUS FOLIATED SPACES
}

\author{
J. A. ÁLVAREZ LÓPEZ* AND A. CANDEL ${ }^{\dagger}$
}

\section{Contents}

Introduction
1. Foliated spaces
2. Pseudogroups of local transformations
3. Coarse quasi-isometries
4. Coarse quasi-isometry type of orbits
5. Fölner orbits
6. An example of non-recurrent compact generation
7. Quasi-local metric spaces
8. Equicontinuous pseudogroups
9. Quasi-effective pseudogroups
10. Coarse quasi-isometry type of orbits with trivial groups of germs
11. Minimality of the orbit closures
12. The closure of a strongly equicontinuous pseudogroup
13. Local metric spaces
14. Pseudogroups of local isometries
15. Isometrization of strongly equicontinuous pseudogroups
16. A non-standard description of weak equicontinuity
17. Strongly equicontinuous foliated spaces
References

\section{INTRODUCTION}

The theme of this paper originates in the study of the generic geometry of leaves of a foliated space. In [3], we analyze the problem of when all (or almost all) the leaves of a foliated space are quasi-isometric. In this paper, a dynamical condition on a foliated space guaranteeing that all the leaves without holonomy are quasi-isometric will be discussed. The condition is on the structure of the action of the holonomy pseudogroup, and is called equicontinuity. That such condition may imply that all leaves without holonomy are quasiisometric comes from the structure theorem of Riemannian foliations. These foliations are the models of equicontinuous foliated spaces, and P. Molino's work [17] describing their structure has as a consequence that the holonomy covers of all the leaves are quasiisometric via a diffeomorphism. Indeed, given a compact, connected manifold $M$ endowed with a Riemannian foliation, Molino shows that there is a fiber bundle over $M$ with compact fiber (the transverse orthonormal frame bundle), and with a foliation transverse to the fibers

\footnotetext{
*Research of the first author supported by DGICYT Grant PB95-0850.

${ }^{\dagger}$ Research of the second author supported by NSF Grants.
} 
whose leaves are the holonomy covers of the leaves of $M$. Furthermore, there is a group of automorphisms of this bundle which permutes the leaves.

The concept of Riemannian foliation is easily formulated by saying that the holonomy pseudogroup is a pseudogroup of local isometries of a Riemannian manifold. This generalizes to equicontinuous pseudogroups of local transformations of topological spaces. As such, the concept of equicontinuity appears in R. Sacksteder's paper [24]. The parallelism between Riemannian foliations and equicontinuous pseudogroups is developed by E. Ghys in [17, Appendix E], see also M. Kellum's paper [16] for this connection.

Thus, in certain measure, what is done in this paper may be seen as a generalization of that aspect of Molino's theory pointed out above. First we show that all leaves without holonomy have the same coarse quasi-isometry type. For general equicontinuous foliated spaces, it seems not to be possible to obtain this result, the main obstruction being the very general structure of the transverse models. This obstruction can be overcome by imposing certain regularity to the transverse structure. The proof of this result requires certain amount of work on pseudogroups and on the geometric structure of their orbits, and on how to pass from coarse quasi-isometries between orbits to leaves.

When the foliated space is smooth, then it is possible to introduce a metric tensor on the leaves that varies continuously from leaf to leaf. In this case the above results can be improved by using quasi-isometries via diffeomorphisms between leaves. Moreover, for general equicontinuous foliated spaces, it can be shown that the universal covers of all leaves are quasi-isometric to each other via diffeomorphisms. These results are obtained with the help of normal bundle theory.

From our study of pseudogroups, it also follows that equicontinuous foliated spaces (with some mild conditions) satisfy two other typical properties of Riemannian foliations. First, the leaf closures are homogeneous spaces and form a partition. Second, the holonomy pseudogroup is indeed given by local isometries with respect to some "local metric," and has a closure in certain sense. The existence of this closure of the holonomy pseudogroup is an important ingredient of our topological description of Riemannian foliations with dense leaves given in [2].

The main results of this paper and [2] were conjectured and greatly justified by E. Ghys [17, Appendix E]. The concept of equicontinuity for foliations was also studied by M. Kellum [16] and C. Tarquini [27]. Kellum dealt with the more restrictive setting of transversely quasi-isometric foliations, and Tarquini showed that equicontinuous transversely conformal foliations are Riemannian, which also follows from our main result of [2] in the case of dense leaves.

Acknowledgments. The work of the first author was supported by DGICYT Grant PB95-0850, and that of the second by NSF Grants No. DMS-9973086 and DMS-0049077. The authors were at the Universidade de Santiago de Compostela and at CSUN while this paper was being written, and thank these institutions for their support. We also thank F. Alcalde Cuesta for helpful conversations.

\section{Foliated SPACES}

This section collects and develops some information on foliated spaces which will be used later. General references on foliated spaces are [11], [18], [5].

The definition of the concept of foliated space (or lamination) requires that of smooth function. Let $Z$ be a Polish space (i.e., a completely metrizable separable space) and let $U$ be an open set in $\mathbb{R}^{n} \times Z$. A map $f: U \rightarrow \mathbb{R}^{p}$ is smooth (of class $C^{k}$ ) at the point $\left(x_{0}, z_{0}\right)$ if there is a neighborhood of $\left(x_{0}, z_{0}\right)$ in $U$ of the form $D \times Y$ (where $D$ is an open ball in $\mathbb{R}^{n}$ 
and $Y$ is an open subset of $Z$ ) such that $f$ is continuous on $D \times Y$ and the partial derivatives up to order $\leq k$ of all coordinate functions $f_{i}$ of $f$ exist and are continuous at all points $(x, z) \in D \times Y$.

A locally compact Polish space $X$ is said to have the structure of a foliated space (of class $\left.C^{k}\right)$ if there is a collection of charts (flow boxes) $\left(U_{i}, \phi_{i}\right)$, where $\left\{U_{i}\right\}$ is a covering of $X$ by open sets, the maps $\phi_{i}$ are homeomorphisms $\phi_{i}: U_{i} \rightarrow B_{i} \times Z_{i}$, for Polish spaces $Z_{i}$ and open balls $B_{i}$ of finite radius in $\mathbb{R}^{n}$, such that the coordinate changes are of the form

$$
\phi_{i} \circ \phi_{j}^{-1}\left(x_{j}, z_{j}\right)=\left(x_{i}\left(x_{j}, z_{j}\right), z_{i}\left(z_{j}\right)\right) \text {, }
$$

where $x_{i}: \phi_{j}\left(U_{i} \cap U_{j}\right) \rightarrow \mathbb{R}^{n}$ is of class $C^{k}$ and $z_{i}$ is continuous. The sets $\phi_{i}^{-1}\left(B_{i} \times\{z\}\right)$ are called plaques.

Since the foliated space $X$ is locally compact, given an open cover $\mathcal{U}=\left\{U_{i}, \phi_{i}\right\}$ by flow boxes of $X$, it is always possible to find a locally finite covering $\left\{V_{\alpha}, \varphi_{\alpha}\right\}$ by flow boxes which is a refinement of $\mathcal{U}$ in the sense that each $V_{\alpha}$ has compact closure on some $U_{i(\alpha)}$ and the corresponding chart $\phi_{i(\alpha)}$ extends $\varphi_{\alpha}$. Such cover $\left\{V_{\alpha}\right\}$ is called regular [11], [5].

A synonym of the term foliated space is lamination, which is sometimes reserved for a foliated space which is embedded as a closed subset of a manifold. It may also be convenient to denote the foliated space by $(X, \mathcal{F})$, with $\mathcal{F}$ referring to the particular foliated structure on $X$.

The foliated structure of a space $X$ induces a locally euclidean topology on $X$, the basic open sets being the open subsets of the plaques, which is finer than the original. The connected components of $X$ in this topology are called leaves. The smooth structure implies that each leaf is a connected manifold of dimension $n$ and of class $C^{k}$. If $x$ is a point of $X$, the leaf which contains $x$ will usually be denoted by $L_{x}$.

Concepts of manifold theory readily extend to foliated spaces. In particular, if $\mathcal{F}$ is at least of class $C^{1}$, there is a continuous vector bundle $T X$ over $X$ whose fiber at each point $x$ of $X$ is the tangent space of the manifold $L_{x}$ at $x$.

Some very basic properties of foliated spaces will now be listed. They easily follow from the definition and standard techniques of manifold theory extended to "manifolds with parameters." A basic observation is the following [18], which is immediate from the paracompactness of Polish spaces and the local structure of foliated spaces.

Proposition 1.1. Every open cover of a foliated space of class $C^{k}$ admits a subordinate partition of unity of class $C^{k}$.

The important consequence is that a foliated space of class $C^{k}$, with $k \geq 1$, always admits a metric tensor; i.e., and inner product on $T X$ inducing a Riemannian metric on each leaf, and varying continuously from leaf to leaf. The induced distance on a leaf $L$ will be denoted by $d_{L}$. If $X$ is compact, then each leaf is a complete Riemannian manifold of bounded geometry. (This may not be the case in general.)

Another consequence of the existence of partitions of unity is the following. The proof is like that for manifolds [5].

Proposition 1.2. If $X$ is a compact foliated space of class $C^{k}, k \geq 1$, then there is a $C^{k}$ embedding $\varphi$ of $X$ in the separable real Hilbert space $\mathbb{E}$. Moreover, a given metric tensor along the leaves can be extended to a metric tensor on $\mathbb{E}$.

A given metric tensor along the leaves of $X$ admits an extension to a metric tensor on $\mathbb{E}$. This is so because a metric tensor may be viewed as a section of some bundle over $\mathbb{E}$ with contractible fibers, and $X$ being closed in $\mathbb{E}$ implies that a section over $X$ extends to one over $\mathbb{E}$. It may only be continuous, of course, but that is sufficient to define length. 
If $X$ is compact, then any two metric tensors on the leaves of $X$ are quasi-isometric, and so, for the purposes of this paper, the metric tensor induced from the standard metric of $\mathbb{E}$ will be taken by default.

This embedding of a foliated space $X$ in $\mathbb{E}$ also gives rise to a normal vector bundle, the fibers of which are isomorphic to $\mathbb{E}$. This structure permits to formalize concepts and results like "local projection of leaves onto leaves" or "Reeb's stability." This structure will be described presently.

Let $X$ be a compact foliated space, of class $C^{k}$ with $k \geq 1$, embedded in the Hilbert space $\mathbb{E}$. The restriction of the embedding to each leaf is not an embedding, but only an injective immersion. The smoothness of $X$ being at least $C^{1}$ implies that the map which assigns to a point $x \in X$ the subspace $T_{x} X$ of $\mathbb{E}$ is continuous (as a map of $X$ into the space of $n$-dimensional subspaces of $\mathbb{E}$ ). It follows that if $F$ is a subspace complementary to one $T_{x} X$ in $\mathbb{E}$, then it is also complementary to $T_{y} X$ for $y$ close to $x$.

The key point is that each tangent space $T_{x} X$ is a finite dimensional subspace of $\mathbb{E}$, hence is closed and has an orthogonal complement. If $i: L \rightarrow \mathbb{E}$ is the inclusion of a leaf of $X$ in $\mathbb{E}$, then there are charts about $x$ in $L$ and $\mathbb{E}$ such that the corresponding local representation of $i$ is of the form $y \mapsto(y, 0)$, provided by the implicit function theorem (flat coordinates). In these flat coordinates, $T_{y} X^{\perp}=\{y\} \times \mathbb{E}$ for $y$ in this plaque containing $x$, and by continuity, the affine subspaces $y+T_{y} X^{\perp}$ meet nearby leaves transversely. Since $X$ is compact, it follows that this holds for all $y$ in a neighborhood of radius $r$ about $x$ in $X$, the radius $r$ being independent of the point $x$.

Theorem 1.3. Let $X$ be a compact foliated space embedded in $\mathbb{E}$ as above, of class $C^{2}$. Let $i: L \rightarrow \mathbb{E}$ denote the inclusion of a leaf $L$ in $X \subset \mathbb{E}$. Then there is a vector bundle $p: N \rightarrow L$ and a neighborhood $W$ of the zero section of $N$ such that the following hold:

(1) The map $i: L \rightarrow \mathbb{E}$ extends to a local diffeomorphism $\varphi: W \rightarrow \mathbb{E}$;

(2) there is a foliated space $Y \subset W$, of the same dimension as $X$, having $L$ as a leaf and transverse to the fibers of $N$; and

(3) as foliated spaces, $Y=\varphi^{-1}(X \cap \varphi(W))$ and the restriction of $p$ to each leaf of $Y$ is a local diffeomorphism into $L$.

Let $N(X)=\left\{(x, v) \mid v \perp T_{x} X\right\} \subset X \times \mathbb{E}$. Then the exponential map $\lambda: N(X) \rightarrow \mathbb{E}$ is $\lambda(x, v)=x+v$. Let $N(X, \varepsilon)$ denote the set of pairs $(x, v) \in N(X)$ with $\|v\|<\varepsilon$. The normal bundle $N(L)$ to each leaf $L$ is contained in $N(X)$.

The differential $\lambda_{*}$ at each point $(x, 0) \in N(X)$ is the identity (under the canonical identification $\left.T_{(x, 0)} N(X)=T_{x} X \times T_{x} X^{\perp}\right)$. Therefore, by continuity of $\lambda_{*}$ on $N(X)$ and compactness of $X$, there exists $\varepsilon>0$ such that $\lambda_{*}$ is an isomorphism at $(x, v)$ for $x \in X$ and $\|v\|<\varepsilon$. This does not mean that $\lambda$ is locally a homeomorphism. What it means is the following:

Lemma 1.4. For each point $x \in X$ there exists a neighborhood $U_{x}$ in the leaf through $x$ such that $\lambda$ is a diffeomorphism of $N\left(U_{x}, \varepsilon\right)$ into $\mathbb{E}$.

It will be shown that there exists $\varepsilon>0$ and $r>0$ such that $\lambda$ is a diffeomorphism of $N(B(x, r), \varepsilon)$ into $\mathbb{E}$, for every $x \in X$. Otherwise, by compactness of $X$, there exist sequences $\left(x_{n}, v_{n}\right) \neq\left(y_{n}, w_{n}\right)$ in $N(X)$, with $\left\|v_{n}\right\|,\left\|w_{n}\right\| \rightarrow 0, x_{n}, y_{n} \rightarrow x$, and such that $\lambda\left(x_{n}, v_{n}\right) \neq \lambda\left(y_{n}, w_{n}\right)$ for each $n$. Working on a local flow box around $x$ in $X$, choosing flat coordinates around the plaque through $x$, and taking into account that the normal subspace to points of $X$ varies continuously, it is then obvious that it is possible to find new sequences $\left(x_{n}^{\prime}, v_{n}^{\prime}\right)$ and $\left(y_{n}^{\prime}, w_{n}^{\prime}\right)$ as above with the added property that all the points $x_{n}^{\prime}$ and $y_{n}^{\prime}$ are in the same plaque as $x$. This contradicts Lemma 1.4 
The vector bundle $N(X)$ has a metric on the fibers so that the continuous map $\phi(x, v)=$ $\lambda(x, v)-x$ is a linear isometry on each fiber. By compactness of $X$, there exists $\varepsilon_{0}>0$ such that for each leaf $L$ of $X$ the restricted map $\lambda: N\left(L, \varepsilon_{0}\right) \rightarrow \mathbb{E}$ is a local diffeomorphism. Moreover, there exists $r>0$ such that for every leaf $L$ and every point $x \in L$, the map $\lambda: N\left(B(x, r), \varepsilon_{0}\right) \rightarrow \mathbb{E}$ is a diffeomorphism.

It follows that if $L$ is a leaf of $X$, then the neighborhood $N\left(L, \varepsilon_{0}\right)$ contains a foliated space $Y$, which is lifted from $X$ via the local diffeomorphism $\lambda$. This completes the proof of Theorem 1.3 .

Remark. The hypothesis that $X$ be of class $C^{2}$ is required so that the normal bundle and exponential map be of class $C^{1}$. On the other hand, a manifold of class $C^{1}$ admits a compatible structure of class $C^{r}$, for any $1 \leq r \leq \infty$. The proof (see [12, Chapter 2]) is based on approximation results that can be adapted to 'manifolds with parameters', that is, to foliated spaces. Since it is out of place to do this here, the $C^{2}$ hypothesis is kept here and in Theorems 17.2, 17.3 .

Remark. Let $x \in L$, and let $T$ be a transversal through $x$, which may be taken to lie in the affine subspace $x+T_{x} X^{\perp}$ of $\mathbb{E}$. If $\mathcal{G}(x)$ denotes the group of germs of local homeomorphisms of $T$ which fix $x$, then there is the germinal holonomy representation

$$
\pi_{1}(L, x) \rightarrow \mathcal{G}(x) .
$$

The construction above shows that this representation is equivalent to that of $L$ as a leaf of the foliated space $Y$ of $N\left(L, \varepsilon_{0}\right)$.

The following two propositions complement Theorem 1.3

Proposition 1.5. Let $L$ be a leaf of the compact foliated space of Theorem 1.3. Then there exists $\varepsilon>0$ and an $\varepsilon$-disc bundle $W \rightarrow L$ which carries a foliated space $Y$ and there exists a constant $K>0$ such that the projection $p: S \rightarrow$ Lo each leaf $S$ of $Y$ into $L$ is a local diffeomorphism (of class $C^{1}$ ) of distortion in the interval $[1 / K, K]$.

The disk bundle $W \rightarrow L$ and foliated space $Y \subset W$ with the stated properties have been constructed in the previous proof. Because the space $X$ is compact, it admits a finite regular cover by flow boxes. The projection $Y \rightarrow L$ amounts to finitely many projections between plaques of these flow boxes. As the cover is finite and regular, there are global bounds for the distortion of these projections within flow boxes. The claim made in the proposition follows from these observations.

A similar useful fact is the following.

Proposition 1.6. Let $L$ be as in Proposition 1.5. Given $R>0$, there exists $\delta>0$ such that, if $x \in L$ and $y$ is a point of $W$ with $p(y)=x$, and such that the distance in the fiber of $W$ between $x$ and $y$ is $<\delta$, then the ball $B(y, R)$ in the leaf of $Y$ through $y$ is contained in $W$.

Proof. The proof of this fact uses again a finite regular cover by flow boxes. The local coordinate changes in the transverse direction are uniformly continuous maps, and only a finite number of coordinate changes are required to run through a ball of radius $R$, because plaques have a definite size.

Remark. The construction of Theorem 1.3 and the discussion that follows it (especially Proposition 1.5, extend to a covering $\pi: L^{\prime} \rightarrow L$ of a leaf $L$, simply by considering the normal bundle of the immersion $L^{\prime} \rightarrow L \hookrightarrow \mathbb{E}$. The output is a disc bundle $W \rightarrow L^{\prime}$ containing a foliated space $Y$ transverse to the fibers and having $L^{\prime}$ as a leaf. The projection of leaves of $Y$ into $L$ is a local diffeomorphism of bounded distortion. 
Continuing with the notation so far introduced, let $L^{\prime} \rightarrow L$ be a covering of a leaf $L$ of $X$, let $x \in L^{\prime}$ and let $D$ be a compact manifold with boundary. Let $y \in D$ and let $f: D \rightarrow L^{\prime}$ be a continuous map with $f(y)=x$. The following is a Reeb stability type of result.

Proposition 1.7. Suppose that the induced homomorphism $\pi_{1}(D, y) \rightarrow \pi_{1}\left(L^{\prime}, x\right)$ takes $\pi_{1}(D, x)$ into the kernel of the holonomy representation of $L^{\prime}$ (as a leaf of the foliated space $Y)$. Then there is a transversal $Z \subset Y$ through $x$ and a smooth map $F$ from the product foliation $D \times Z$ into $X$ such that $F \mid D \times\{x\}=f$ and $F(D \times\{y\})$ is contained in the leaf through $y$, for all $y \in Z$.

If $f: D \hookrightarrow L$ is an embedding, $Z$ can be chosen so that $F$ embeds $D \times Z$ in $Y$. In particular, if $\pi_{1}\left(L^{\prime}, x\right) \rightarrow \pi_{1}(L)$ has image contained in the kernel of the holonomy representation of $L$ as a leaf of $X$, then, given $x \in L^{\prime}$ and $R>0$ arbitrarily large, there exists $\delta>0$ such that if $y$ is a point in the fiber $p^{-1}(x)$ at distance $<\delta$ from $x$, then the ball $B(y, K R)$ contains $p^{-1}(B(x, R)) \cap Y$, and the component of this last set which contains $y$, contains the ball $B(y, R / K)$.

Moreover, the absence of holonomy permits to choose $\delta$ so that there is a transversal $Z$ in the fiber through $x$ such that the union of the leaves of $p^{-1}(B(x, R)) \cap Y$ through points of $Z$ is parametrized as a product $B(x, R) \times Z$, and the induced metric on the leaves is at bounded distance from that of $B(x, R)$.

\section{Pseudogroups of local transformations}

A pseudogroup of local transformations of a topological space $Z$ is a collection $\mathcal{H}$ of homeomorphisms between open subsets of $Z$ that contains the identity on $Z$ and is closed under composition (wherever defined), inversion, restriction and combination of maps. Such pseudogroup $\mathcal{H}$ is generated by a set $E \subset \mathcal{H}$ if every element of $\mathcal{H}$ can be obtained from $E$ by using the above pseudogroup operations; to simplify arguments, the sets of generators to be considered will be assumed to be symmetric $\left(h^{-1} \in E\right.$ if $\left.h \in E\right)$.

The orbit of an element $x \in Z$ is the set $\mathcal{H}(x)$ of elements $h(x)$, for all $h \in \mathcal{H}$ whose domain contains $x$. These orbits are the equivalence classes of an equivalence relation on $Z$. Note that an arbitrary equivalence relation $R \subset Z \times Z$ is defined by a pseudogroup on $Z$ if and only if $R$ is a union of sets $R_{i}, i \in I$, such that the restriction to each $R_{i}$ of both factor projections $Z \times Z \rightarrow Z$ are homeomorphisms onto open subsets. Indeed, take the sets $R_{i}$ to be the graphs of all local transformations in the pseudogroup. Moreover $R$ is defined by a countably generated pseudogroup on $Z$ if and only if $R$ is a countable union of sets $R_{i}$ satisfying the above condition. This follows because a countable set of local transformations of $Z$ gives rise to a countable family of composites with maximal domain.

The set of germs of all transformations in the pseudogroup $\mathcal{H}$ at all points of their domains, endowed with the étale topology, is a topological groupoid, product and inversion being induced by composition and inversion of maps, respectively. Thus, for each $x \in Z$, the set of germs at $x$ of transformations $h \in \mathcal{H}$ with $x \in \operatorname{dom} h$ and $h(x)=x$ is a group called the group of germs at $x$. If $x, y \in Z$ are in the same $\mathcal{H}$-orbit, then the groups of germs at $x$ and $y$ are isomorphic: an isomorphism is given by conjugation with the germ at $x$ of any transformation $g \in \mathcal{H}$ with $x \in \operatorname{dom} g$ and $g(x)=y$. The group of germs of an orbit is therefore well defined, up to isomorphisms, as the group of germs at any point of that orbit. In particular, a distinguished type of orbits are those with trivial group of germs.

Pseudogroups of local transformations must be thought of as natural generalizations of group actions on topological spaces (each group action generates a pseudogroup). But the main example to keep in mind is the holonomy pseudogroup of a foliated space $(X, \mathcal{F})$ 
associated to a regular covering by flow boxes $\left(U_{i}, \phi_{i}\right)$, whose construction is now recalled. If $\phi_{i}: U_{i} \rightarrow B_{i} \times Z_{i}$ for Polish spaces $Z_{i}$ and open balls $B_{i}$ of finite radius in $\mathbb{R}^{n}$, let $p_{i}: U_{i} \rightarrow Z_{i}$ denote the composite of $\phi_{i}$ with the factor projection $\mathbb{R}^{n} \times Z_{i} \rightarrow Z_{i}$; the fibers of these $p_{i}$ are the plaques. If $U_{i}$ meets $U_{j}$, let $Z_{i, j}=p_{i}\left(U_{i} \cap U_{j}\right)$, and regularity of the cover permits to define a homeomorphism $h_{i, j}: Z_{i, j} \rightarrow Z_{j, i}$ such that $p_{j}=h_{i, j} \circ p_{i}$ on $U_{i} \cap U_{j}$ [5, 11]. (If the covering by flow boxes is not regular, one can define generators of a pseudogroup via local sections of the projections $p_{i}$.) Such a collection $\left(U_{i}, p_{i}, h_{i, j}\right)$ is called a defining cocycle for $\mathcal{F}[8,9]$. These $h_{i, j}$ generate a pseudogroup $\mathcal{H}$ of local transformations of $Z=\bigsqcup_{i} Z_{i}$, which is called the holonomy pseudogroup of $(X, \mathcal{F})$ (with respect to the covering $\left.\left(U_{i}, \phi_{i}\right)\right)$.

There is a canonical bijection between the set of leaves and the set of $\mathcal{H}$-orbits, which is given by $L \mapsto \mathcal{H}\left(p_{i}(x)\right)$ if $x \in L \cap U_{i}$. Each $Z_{i}$ can be considered as a local transversal of $\mathcal{F}$ via $\phi_{i}$ and the identification $Z_{i} \equiv\{0\} \times Z_{i} \subset B_{i} \times Z_{i}$. It may be assumed that all of these local transversals are disjoint from each other, and thus that $Z$ is embedded in $X$ as a complete transversal. Each $\mathcal{H}$-orbit injects into the corresponding leaf in this way.

The holonomy groups of the leaves can be defined as the groups of germs of the corresponding orbits. Thus leaves with trivial holonomy group correspond to orbits with trivial group of germs. Moreover, with the same arguments of [11], it follows that, for a general pseudogroup $\mathcal{H}$ of local transformations of a topological space $Z$, if $\mathcal{H}$ has a countable set of generators, then the union of orbits with trivial group of germs is a residual subset of $Z$; in particular, this union is dense in $Z$ if $Z$ is a Polish space.

It is well known that all defining cocycles of a foliated space induce holonomy pseudogroups that are equivalent in the sense given by the following definition; thus the relevant properties of pseudogroups of local transformations of a topological space are those that are invariant by these equivalences.

Definition 2.1 (Haefliger [8, 9]). Let $\mathcal{H}, \mathcal{H}^{\prime}$ be pseudogroups of local transformations of topological spaces $Z, Z^{\prime}$, respectively. An étale morphism $\Phi: \mathcal{H} \rightarrow \mathcal{H}^{\prime}$ is a maximal collection $\Phi$ of homeomorphisms of open subsets of $Z$ to open subsets of $Z^{\prime}$ such that:

- If $\phi \in \Phi, h \in \mathcal{H}$ and $h^{\prime} \in \mathcal{H}^{\prime}$, then $h^{\prime} \circ \phi \circ h \in \Phi$;

- the sources of elements of $\Phi$ form a covering of $Z$; and

- if $\phi, \psi \in \Phi$, then $\psi \circ \phi^{-1} \in \mathcal{H}^{\prime}$.

An étale morphism $\Phi: \mathcal{H} \rightarrow \mathcal{H}^{\prime}$ is called an equivalence if the collection $\Phi^{-1}=\left\{\phi^{-1} \mid \phi \in\right.$ $\Phi\}$ is also an étale morphism $\mathcal{H}^{\prime} \rightarrow \mathcal{H}$, which is called the inverse of $\Phi$. The composite of two étale morphisms $\Phi: \mathcal{H} \rightarrow \mathcal{H}^{\prime}$ and $\Psi: \mathcal{H}^{\prime} \rightarrow \mathcal{H}^{\prime \prime}$ is the collection $\Psi \circ \Phi=\{\psi \circ \phi \mid \phi \in$ $\Phi, \psi \in \Psi\}$, which is an étale morphism $\mathcal{H} \rightarrow \mathcal{H}^{\prime \prime}$. Finally, an étale morphism $\Phi: \mathcal{H} \rightarrow \mathcal{H}^{\prime}$ is generated by a subset $\Phi_{0} \subset \Phi$ if all the elements of $\Phi$ can be obtained by restriction and combination of composites $h^{\prime} \circ \phi \circ h$ with $h \in \mathcal{H}, \phi \in \Phi_{0}$ and $h^{\prime} \in \mathcal{H}^{\prime}$.

An étale morphism $\Phi: \mathcal{H} \rightarrow \mathcal{H}^{\prime}$ clearly induces a continuous map between the corresponding spaces of orbits, $\bar{\Phi}: Z / \mathcal{H} \rightarrow Z^{\prime} / \mathcal{H}^{\prime}$, which is a homeomorphism if $\Phi$ is an equivalence.

A basic example of a pseudogroup equivalence is the following. Let $\mathcal{H}$ be a pseudogroup of local transformations of a space $Z$, let $U \subset Z$ be an open subset that meets every $\mathcal{H}$-orbit, and let $\mathcal{G}$ denote the restriction of $\mathcal{H}$ to $U$. Then the inclusion map $U \hookrightarrow Z$ generates an equivalence $\mathcal{G} \rightarrow \mathcal{H}$. In fact, this example can be used to describe any pseudogroup equivalence in the following way. Let $\mathcal{H}, \mathcal{H}^{\prime}$ be pseudogroups of local transformations of spaces $Z, Z^{\prime}$, and $\Phi: \mathcal{H} \rightarrow \mathcal{H}^{\prime}$ an equivalence. Then let $\mathcal{H}^{\prime \prime}$ be the pseudogroup of local 
transformations of $Z^{\prime \prime}=Z \sqcup Z^{\prime}$ generated by $\mathcal{H} \cup \mathcal{H}^{\prime} \cup \Phi$. Then the inclusions of $Z, Z^{\prime}$ in $Z^{\prime \prime}$ generate equivalences $\Psi_{1}: \mathcal{H} \rightarrow \mathcal{H}^{\prime \prime}$ and $\Psi_{2}: \mathcal{H}^{\prime} \rightarrow \mathcal{H}^{\prime \prime}$ so that $\Phi=\Psi_{2}^{-1} \circ \Psi_{1}$.

For pseudogroups of local transformations of locally compact spaces, the following result characterizes the existence of relatively compact open subsets that meet all orbits.

Lemma 2.2. Let $\mathcal{H}$ be a pseudogroup of local transformations of a locally compact $Z$. The orbit space $Z / \mathcal{H}$ is compact if and only if there exists a relatively compact open subset that meets every $\mathcal{H}$-orbit.

Proof. If an open subset $U \subset Z$ meets every $\mathcal{H}$-orbit, then the restriction $U \rightarrow Z / \mathcal{H}$ of the quotient map $Z \rightarrow Z / \mathcal{H}$ is onto. Thus $Z / \mathcal{H}$ is compact because it is a continuous image of the compact space $\bar{U}$.

Assume that $Z / \mathcal{H}$ is compact. Since $Z$ is locally compact, each $x \in Z$ has relatively compact open neighborhood $U_{x}$. Let $Q_{x}$ denote the image of $U_{x}$ by the quotient map $Z \rightarrow Z / \mathcal{H}$. Since $Z / \mathcal{H}$ is compact, its open covering $\left\{Q_{x} \mid x \in Z\right\}$ has a finite sub-covering $Q_{x_{1}}, \ldots, Q_{x_{m}}$. Then the open set $U=U_{x_{1}} \cup \cdots \cup U_{x_{n}}$ of $Z$ is relatively compact and meets all orbits of $\mathcal{H}$.

Examples of pseudogroups with compact space of orbits are the holonomy pseudogroups of compact foliated spaces, as orbit and leaf spaces can be identified. But such pseudogroups satisfy a stronger compactness condition that is defined as follows.

Definition 2.3 (Haefliger [8]). Let $\mathcal{H}$ be a pseudogroup of local transformations of a locally compact space $Z$. Then $\mathcal{H}$ is compactly generated if there is a relatively compact open set $U$ in $Z$ meeting each orbit of $\mathcal{H}$, and such that the restriction $\mathcal{G}$ of $\mathcal{H}$ to $U$ is generated by a finite symmetric collection $E \subset \mathcal{G}$ so that each $g \in E$ is the restriction of an element $\bar{g}$ of $\mathcal{H}$ defined on some neighborhood of the closure of the source of $g$.

It was observed in [8] that this notion is invariant by equivalences and that the relatively compact open set $U$ meeting each orbit can be chosen arbitrarily. If $E$ satisfies the conditions of Definition 2.3, it will be called a system of compact generation of $\mathcal{H}$ on $U$.

\section{COARSE QUASI-ISOMETRIES}

The concept of coarse quasi-isometry was introduced by M. Gromov in [7]; it is also called rough isometry in the context of potential theory [15]. A net in a metric space $M$, with metric $d$, is a subset $A \subset M$ such that $d(x, A)<C$ for some $C>0$ and all $x \in M$; the term $C$-net is also used. A coarse quasi-isometry between $M$ and another metric space $M^{\prime}$ is the choice of a bi-Lipschitz bijection between nets of $M$ and $M^{\prime}$; in this case, $M$ and $M^{\prime}$ are said to have the same coarse quasi-isometry type or to be coarsely quasi-isometric. This definition involves two constants that will be called coarse distortions: one for the nets and another one for the bi-Lipschitz equivalence. A collection of coarse quasi-isometries is said to be uniform when the same coarse distortions are valid for all of them.

Recall that the Hausdorff distance between subspaces $X, Y$ of some metric space with metric $d$, is defined as

$$
d_{H}(X, Y)=\max \left\{\sup _{x \in X} d(x, Y), \sup _{y \in Y} d(y, X)\right\} .
$$

Now let $M, M^{\prime}$ be arbitrary metric spaces with metrics $d, d^{\prime}$. The Gromov-Hausdorff distance (also called abstract Hausdorff distance) between $M, M^{\prime}$, denoted by $d_{G H}\left(M, M^{\prime}\right)$, is the infimum of the Hausdorff distances $d_{H}\left(M, M^{\prime}\right)$ over all metrics on $M \sqcup M^{\prime}$ that restrict to $d, d^{\prime}$ on $M, M^{\prime}$. Note that $d_{G H}\left(M, M^{\prime}\right)$ may be equal to $\infty$. If $d_{G H}\left(M, M^{\prime}\right)<\infty$, 
then $M, M^{\prime}$ are called Hausdorff equivalent. On the other hand, the metric spaces $M, M^{\prime}$ are called Lipschitz equivalent when there exists a bi-Lipschitz bijection $M \rightarrow M^{\prime}$. Then $M, M^{\prime}$ are coarsely quasi-isometric if and only if there are some metric spaces $N, N^{\prime}$ such that the pairs $M, N$ and $M^{\prime}, N^{\prime}$ are Hausdorff equivalent, and $N, N^{\prime}$ are Lipschitz equivalent.

There is also a categorical description of coarse quasi-isometries. Two maps $f, g: M \rightarrow$ $M^{\prime}$ are called parallel [7], or bornotopic [23] or uniformly close [4] if there is some $R>0$ such that $d^{\prime}(f(x), g(x))<R$ for all $x \in M$. A map $f: M \rightarrow M^{\prime}$ is said to be large scale Lipschitz [7] if there are constants $\lambda, c>0$ such that

$$
d^{\prime}(f(x), f(y)) \leq \lambda d(x, y)+c
$$

for all $x, y \in M$; note that $f$ need not be continuous. Then coarse quasi-isometries can be considered as isomorphisms in the category of metric spaces and parallel classes of large scale Lipschitz maps.

The above description of coarse quasi-isometry is similar to the definition of another type of "coarse" equivalence. A map $f: M \rightarrow M^{\prime}$ is called effectively proper [4] if for all $r>0$ there is some $s>0$ so that

$$
d^{\prime}(f(x), f(y))<r \Longrightarrow d(x, y)<s
$$

for all $x, y \in M$. The map $f$ is called uniformly bornologous [23] or (coarsely) Lipschitz [4] if for all $r>0$ there is some $s>0$ so that

$$
d(x, y)<r \Longrightarrow d^{\prime}(f(x), f(y))<s
$$

for all $x, y \in M$. Then two metric spaces are called uniformly close [4] if there is an isomorphism between them in the category of metric spaces and uniformly close classes of effectively proper coarsely Lipschitz maps. Note that every large scale Lipschitz map is coarsely Lipschitz, and it is also effectively proper if it has a large scale Lipschitz inverse up to the uniform closeness of maps. Therefore coarsely quasi-isometric metric spaces are uniformly close.

Uniform closeness of metric spaces is a slight modification of the concept of bornotopy equivalence introduced in [23], which is an isomorphism in the category of proper metric spaces and bornotopy classes of effectively proper uniformly bornologous Borel maps. Here, a metric space is called proper when its closed bounded subsets are compact. Thus bornotopy equivalence is the same as uniform closeness for all spaces that will be considered in this paper.

\section{COARSE QUASI-ISOMETRY TYPE OF ORBITS}

Let $\mathcal{H}$ be a pseudogroup of local transformations of a space $Z$, and $E$ a symmetric set of generators of $\mathcal{H}$. For each $h \in \mathcal{H}$ and each $x \in \operatorname{dom} h$, let $|h|_{E, x}$ be defined as follows. If $h$ is the identity around $x$, set $|h|_{E, x}=0$. Otherwise, $|h|_{E, x}$ is the minimal positive integer $k$ such that $h=h_{k} \circ \cdots \circ h_{1}$ around $x$ for some $h_{1}, \ldots, h_{k} \in E$. Let $R \subset Z \times Z$ denote the equivalence relation induced by $\mathcal{H}$ (whose equivalence classes are the orbits). Then, for $(x, y) \in R$, let

$$
d_{E}(x, y)=\min \left\{|h|_{E, x} \mid h \in \mathcal{H}, x \in \operatorname{dom} h, h(x)=y\right\} .
$$

In this way, $E$ induces a map $d_{E}: R \rightarrow \mathbb{N}$ whose restriction to each orbit is a metric. This is a well known construction of a metric on the orbits; especially, for group actions.

Unlike the case of group actions, for a pseudogroup $\mathcal{H}$ of local transformations of a space $Z$ with a symmetric set $E$ of generators, the coarse quasi-isometry type of the induced metric $d_{E}$ on the orbits may depend on the choice of $E$, even if $E$ is finite. This is due to the fact that not only composition of maps is used to generate a group action, but 
restriction and combination of maps are also used to generate $\mathcal{H}$. Moreover, an equivalence of pseudogroups may not preserve the coarse quasi-isometry type of the orbits for any choice of generators, as can be seen in the following simple example.

Example 4.1. Let $\mathcal{H}$ be the pseudogroup on $\mathbb{R}$ generated by the action of $\mathbb{Z}$ by translations, and let $\mathcal{G}$ be the restriction of $\mathcal{H}$ to some open interval $U \subset \mathbb{R}$. If $U$ is of length $>1$, then it meets every $\mathcal{H}$-orbit, and thus $\mathcal{H}$ is equivalent to $\mathcal{G}$. But the $\mathcal{H}$-orbits are infinite, while each $\mathcal{G}$-orbit is finite if $U$ is of bounded length. So, for the metrics induced by any choice of symmetric families of generators of $\mathcal{H}, \mathcal{G}$, the $\mathcal{H}$-orbits have infinite diameter and the $\mathcal{G}$-orbits finite diameter, and thus cannot be coarsely quasi-isometric.

In the measure theoretic setting, this problem is solved by considering Kakutani equivalences [14], which are kind of measure theoretic counterparts of étale equivalences with the additional requirement that the coarse quasi-isometry type of the orbits is preserved. In the present topological context, the above problem will be addressed without adding more conditions to étale equivalences. Instead, appropriate representatives of pseudogroups and sets of generators will be chosen to determine a coarse quasi-isometry type on the orbits. The choice of appropriate pseudogroup representatives is easy, while the choice of appropriate generators is rather delicate.

Let $\mathcal{H}$ be a pseudogroup of local transformations of a locally compact space $Z$ with compact orbit space. By Lemma 2.2 there is a relatively compact open subset $U$ of $Z$ that meets all $\mathcal{H}$ orbits. If $\mathcal{H}$ is indeed compactly generated, the restriction $\mathcal{G}$ of $\mathcal{H}$ to $U$ is a representative of $\mathcal{H}$ whose orbits will be shown to have a canonical coarse quasi-isometry type, which is determined by any symmetric set $E$ of generators of $\mathcal{G}$ satisfying certain conditions. The first condition on $E$ is that it must be a system of compact generation of $\mathcal{H}$ on $U$. But this is not enough because there are systems of compact generation on the same open set inducing different coarse quasi-isometry types in the same orbit; such an example will be given in Section 6. So a second new condition is introduced as follows.

Definition 4.2. A finite symmetric family $E$ of generators of a pseudogroup $\mathcal{H}$ of local transformations of a locally compact space $Z$ is said to be recurrent if there exists a relatively compact open subset $U \subset Z$ and some $R>0$ such that any $d_{E}$-ball of radius $R$ in any $\mathcal{H}$-orbit meets $U$; i.e., for any $x \in Z$ there exists $h \in \mathcal{H}$ with $x \in \operatorname{dom} h,|h|_{E, x}<R$ and $h(x) \in U$.

The role played by $U$ in Definition 4.2 can actually be played by any relatively compact open subset that meets all orbits, as shown by the following result.

Lemma 4.3. Let $\mathcal{H}$ be a pseudogroup of local transformations of a locally compact space $Z$, and let $E$ be a recurrent finite symmetric family of generators of $\mathcal{H}$. If $V \subset Z$ is an open set that meets every orbit, then there exists $S>0$ such that any $d_{E}$-ball of radius $S$ in any $\mathcal{H}$-orbit meets $V$.

Proof. By definition, there exist a relatively compact open subset $U \subset Z$ and a positive number $R$ such that any $d_{E}$-ball of radius $R$ in any $\mathcal{H}$-orbit meets $U$. Since $V$ also meets every orbit, there exists a finite family $F \subset \mathcal{H}$ such that:

- the sources of elements of $F$ cover the compact closure $\bar{U}$;

- the targets of elements of $F$ are contained in $V$; and

- each element of $F$ is a composite of elements of $E$.

Let $r>0$ be an integer so that every $g \in F$ can be written as a composition of at most $r$ elements of $E$. 
Fix any $x \in Z$. On the one hand, there is some $h \in \mathcal{H}$ with $x \in \operatorname{dom} h,|h|_{E, x}<R$ and $h(x) \in U$. On the other hand, there is some $g \in F$ whose domain contains $h(x)$. So $x \in \operatorname{dom}(g h), g h(x) \in V$, and

$$
|g h|_{E, x} \leq|g|_{E, h(x)}+|h|_{E, x} \leq r+R .
$$

Thus the result follows with $S=r+R$.

Let $\mathcal{H}$ be a compactly generated pseudogroup of local transformations of a locally compact space $Z$. A system of compact generation of $\mathcal{H}$ on a relatively compact open subset $U \subset Z$ that meets every orbit is called recurrent if it is recurrent when considered as finite symmetric set of generators of the restriction of $\mathcal{H}$ to $U$. An example of a non-recurrent system of compact generation will be given in Section 6 On the other hand, the existence of recurrent systems of compact generation will be a consequence of the following result.

Lemma 4.4. With the above notation, let $E$ be a system of compact generation of $\mathcal{H}$ on $U$. For each $g \in E$, fix an extension $\bar{g} \in \mathcal{H}$ of $g$ with $\overline{\operatorname{dom} g} \subset \operatorname{dom} \bar{g}$. Suppose that every $x \in \bar{U}$ has an open neighborhood $V_{x}$ in $Z$ such that

$$
V_{x} \subset \operatorname{dom} \bar{g}_{x}, \quad V_{x} \cap U \subset \operatorname{dom} g_{x}, \quad \bar{g}_{x}\left(V_{x}\right) \subset U
$$

for some $g_{x} \in E$. Then $E$ is recurrent.

Proof. For each $x \in \bar{U}$, let $W_{x}=\bar{g}_{x}\left(V_{x}\right)$; its closure can be assumed to be contained in $U$. Compactness of $\bar{U}$ implies that $\bar{U} \subset V_{x_{1}} \cup \cdots \cup V_{x_{n}}$, for some finite set of points $x_{1}, \ldots, x_{n} \in \bar{U}$. Let $V_{k}=V_{x_{k}}, W_{k}=W_{x_{k}}$ and $g_{k}=g_{x_{k}}$ for $k=1, \ldots, n$, so $W=W_{1} \cup \cdots \cup W_{n}$ is a relatively compact open set in $U$. Moreover, each $y \in U$ belongs to some $V_{k}$; thus $y \in V_{k} \cap U \subset \operatorname{dom} g_{k}$ and $g_{k}(y) \in W$, yielding $d_{E}(y, W \cap \mathcal{H}(y)) \leq 1$.

Corollary 4.5. Let $\mathcal{H}$ be a compactly generated pseudogroup of local transformations of a locally compact space $Z$, and let $U$ be a relatively compact open subset of $Z$ that meets all $\mathcal{H}$-orbits. Then there exists a recurrent system $E$ of compact generation of $\mathcal{H}$ on $U$ satisfying the following property. The extension $\bar{g} \in \mathcal{H}$ of each $g \in E$ with $\overline{\operatorname{dom} g} \subset \operatorname{dom} \bar{g}$ can be chosen so that $\bar{E}=\{\bar{g} \mid g \in E\}$ is also a recurrent system of compact generation on some relatively compact open subset $U^{\prime} \subset Z$ with $\bar{U} \subset U^{\prime}$.

Proof. Since $U$ meets every $\mathcal{H}$-orbit and $\bar{U}$ is compact, there exists a finite family $F \subset \mathcal{H}$ satisfying the following properties:

- each $f \in F$ is the restriction of some $\bar{f} \in \mathcal{H}$ whose domain is relatively compact and contains $\overline{\operatorname{dom} f}$;

- each $\bar{f}$ is the restriction of some $\tilde{f} \in \mathcal{H}$ with $\overline{\operatorname{dom} \bar{f}} \subset \operatorname{dom} \tilde{f}$;

- the sources of elements of $F$ cover $\bar{U}$; and

- $\operatorname{im} \bar{f} \subset U$ for every $f \in F$.

For each $f \in F$, let $f^{\prime}$ denote its restriction

$$
U \cap \operatorname{dom} f \rightarrow f(U \cap \operatorname{dom} f),
$$

let $F^{\prime}=\left\{f^{\prime} \mid f \in F\right\}$, and set $F^{\prime-1}=\left\{f^{\prime-1} \mid f^{\prime} \in F^{\prime}\right\}$.

There exists a system $G$ of compact generation of $\mathcal{H}$ on $U$, and $E=G \cup F^{\prime} \cup F^{\prime-1}$ is also a system of compact generation of $\mathcal{H}$ on $U$. Moreover, $E$ satisfies the condition of Lemma 4.4 because

$$
\bar{U} \subset \bigcup_{f \in F} \operatorname{dom} f,
$$

and $\operatorname{im} \bar{f} \subset U$ for every $f \in F$. Thus $E$ is recurrent. 
Now let

$$
U^{\prime}=\bigcup_{f \in F} \operatorname{dom} \bar{f},
$$

which is a relatively compact open subset of $Z$ containing $\bar{U}$. Let $\bar{F}=\{\bar{f} \mid f \in F\}$ and $\bar{F}^{-1}=\left\{\bar{f}^{-1} \mid f \in F\right\}$, which are subsets of the restriction $\mathcal{G}^{\prime}$ of $\mathcal{H}$ to $U^{\prime}$. The extensions $\bar{g}$ of the maps $g \in G$ satisfying $\overline{\operatorname{dom} g} \subset \operatorname{dom} \bar{g}$ can obviously be chosen so that:

- each $\bar{g}$ has source and range in $U^{\prime}$;

- the set $\bar{G}=\{\bar{g} \mid g \in G\}$ is symmetric; and

- each $\bar{g}$ is the restriction of some $\tilde{g} \in \mathcal{H}$ with $\overline{\operatorname{dom} \bar{g}} \subset \operatorname{dom} \tilde{g}$.

Then $\bar{E}=\bar{G} \cup \bar{F} \cup \bar{F}^{-1}$ is a finite symmetric subset of $\mathcal{G}^{\prime}$ which generates $\mathcal{G}^{\prime}$ because $G$ generates $\mathcal{G}$ and $\operatorname{im} \bar{f} \subset U$ for all $f \in F$. The above properties guarantee that $\bar{E}$ is a system of compact generation of $\mathcal{H}$ on $U^{\prime}$. Finally, $\bar{E}$ is recurrent by Lemma 4.4 since im $\bar{f} \subset U$ for all $f \in F$.

The following is the promised result that shows the invariance of the coarse quasiisometry type of the orbits by equivalences when appropriate representatives of pseudogroups and generators are chosen.

Theorem 4.6. Let $\mathcal{H}, \mathcal{H}^{\prime}$ be compactly generated pseudogroups of local transformations of locally compact spaces $Z, Z^{\prime}$, and let $U, U^{\prime}$ be relatively compact open subsets of $Z, Z^{\prime}$ that meet all orbits of $\mathcal{H}, \mathcal{H}^{\prime}$, respectively. Let $\mathcal{G}, \mathcal{G}^{\prime}$ denote the restrictions of $\mathcal{H}, \mathcal{H}^{\prime}$ to $U, U^{\prime}$, and let $E, E^{\prime}$ be recurrent symmetric systems of compact generation of $\mathcal{H}, \mathcal{H}^{\prime}$ on $U, U^{\prime}$, respectively. Suppose that there exists an equivalence $\mathcal{H} \rightarrow \mathcal{H}^{\prime}$, and consider the induced equivalence $\mathcal{G} \rightarrow \mathcal{G}^{\prime}$ and homeomorphism $U / \mathcal{G} \rightarrow U^{\prime} / \mathcal{G}^{\prime}$. Then the $\mathcal{G}$-orbits with $d_{E}$ are uniformly coarsely quasi-isometric to the corresponding $\mathcal{G}^{\prime}$-orbits with $d_{E^{\prime}}$.

In other words, Theorem 4.6 asserts that, for pseudogroups $\mathcal{H}$ of local transformations of locally compact spaces $Z$ with given sets of generators $E$, the coarse quasi-isometry type of the orbits is uniformly invariant by equivalences when the following conditions are satisfied. First, $\mathcal{H}$ must be the restriction of a pseudogroup $\mathcal{H}^{\prime}$ acting on a larger locally compact space where $Z$ is open, relatively compact and meets all $\mathcal{H}^{\prime}$-orbits. Second, $E$ must be a recurrent system of compact generation of $\mathcal{H}^{\prime}$ on $Z$.

In order to prove Theorem 4.6, the following preliminary results will be required.

Lemma 4.7. Let $\mathcal{H}$ be a compactly generated pseudogroup of local transformations of a locally compact space $Z$, let $U, U^{\prime}$ be relatively compact open subsets of $Z$ such that $U \cap U^{\prime}$ meets all $\mathcal{H}$-orbits, and let $E, E^{\prime}$ be recurrent systems of compact generation of $\mathcal{H}$ over $U, U^{\prime}$, respectively. Then, for any open set $V$ that meets all $\mathcal{H}$-orbits and with $\bar{V} \subset U \cap U^{\prime}$, there exists some $C>0$ such that

$$
\frac{1}{C} d_{E^{\prime}}(x, y) \leq d_{E}(x, y) \leq C d_{E^{\prime}}(x, y)
$$

for all $x, y \in V$ lying in the same $\mathcal{H}$-orbit.

Proof. Let $\mathcal{G}$ denote the restriction of $\mathcal{H}$ to $U$. By Lemma 4.3 there exists some $R>0$ such that any $d_{E}$-ball of radius $R$ in any $\mathcal{G}$-orbit meets $V$. Let $\Phi \subset \mathcal{G}$ denote the finite set of restrictions of the form

$$
g: V \cap \operatorname{dom} g \rightarrow g(V \cap \operatorname{dom} g),
$$


where $g$ runs over the composites of at most $R$ elements of $E$, wherever defined. It is noted that the images of elements of $\Phi$ cover $U$. Moreover, it may be assumed that $R \geq 2$, and thus that the identity map of $V \cap \operatorname{dom} g$ belongs to $\Phi$ for all $g \in E$ with $V \cap \operatorname{dom} g \neq \emptyset$.

Let $F$ denote the finite set of composites $\psi^{-1} \circ g \circ \phi$, wherever defined, where $\phi, \psi \in \Phi$ and $g \in E$. Observe that each $f \in F$ is the restriction of some $\bar{f} \in \mathcal{G}$ with $\overline{\operatorname{dom} f} \subset$ $\operatorname{dom} \bar{f}$. Furthermore, for each $x \in \operatorname{dom} \bar{f}$, it holds that $|\bar{f}|_{E^{\prime}, y} \leq|\bar{f}|_{E^{\prime}, x}$, for all $y$ in some neighborhood of $x$ in dom $\bar{f}$. Hence, since $F$ is finite and the domain of each $f \in F$ is relatively compact in $U^{\prime}$, there exists an integer $S>0$ such that $|f|_{E^{\prime}, x} \leq S$ for all $f \in F$ and all $x \in \operatorname{dom} f$.

Let $x, y \in V$ be points in the same $\mathcal{H}$-orbit. If $x=y$, then $d_{E}(x, y)=d_{E^{\prime}}(x, y)=0$, and the statement holds trivially with any $C>0$. If $x \neq y$, then $d_{E}(x, y)=k \geq 1$. Let $g \in \mathcal{G}$ be such that $x \in \operatorname{dom} g, g(x)=y$ and $|g|_{E, x}=k$. This element $g$ may be assumed to be of the form $g=g_{k} \circ \cdots \circ g_{1}$ for some $g_{1}, \ldots, g_{k} \in E$. Then, for $i=1, \ldots, k-1$, there exists $\phi_{i} \in \Phi$ whose image contains $x_{i}=g_{i} \circ \cdots \circ g_{1}(x)$ and such that $z_{i}=\phi_{i}^{-1}\left(x_{i}\right) \in V$. Such $g$ can be written as $g=f_{k} \circ \cdots \circ f_{1}$ around $x$, where $f_{1}, \ldots, f_{k}$ are the elements of $F$ given by

$$
f_{1}=\phi_{1}^{-1} \circ g_{1}, \quad f_{k}=g_{k} \circ \phi_{k-1}, \quad f_{i}=\phi_{i}^{-1} \circ g_{i} \circ \phi_{i-1},
$$

for $i=2, \ldots, k-1$. Observe that $x \in \operatorname{dom} f_{1}$ and $z_{i} \in \operatorname{dom} f_{i+1}$ for all $i=1, \ldots, k-1$. Therefore,

$$
|g|_{E^{\prime}, x} \leq\left|f_{k}\right|_{E^{\prime}, z_{k-1}}+\cdots+\left|f_{2}\right|_{E^{\prime}, z_{1}}+\left|f_{1}\right|_{E^{\prime}, x} \leq k S
$$

yielding

$$
d_{E^{\prime}}(x, y) \leq S d_{E}(x, y) .
$$

Similarly,

$$
d_{E}(x, y) \leq S^{\prime} d_{E^{\prime}}(x, y)
$$

for some integer $S^{\prime}>0$, and result follows with $C=\max \left\{S, S^{\prime}\right\}$.

Corollary 4.8. Let $\mathcal{H}$ be a compactly generated pseudogroup of local transformations of a locally compact space $Z$ and let $U, U^{\prime}$ be relatively compact open subsets of $Z$ such that $U \cap U^{\prime}$ meets all $\mathcal{H}$-orbits. Let $\mathcal{G}, \mathcal{G}^{\prime}$ denote the restrictions of $\mathcal{H}$ to $U, U^{\prime}$, and let $E, E^{\prime}$ be recurrent systems of compact generation of $\mathcal{H}$ over $U, U^{\prime}$, respectively. Then the $\mathcal{G}$-orbits with $d_{E}$ are uniformly coarsely quasi-isometric to the corresponding $\mathcal{G}^{\prime}$-orbits with $d_{E^{\prime}}$.

Proof. There exists an open set $V$ meeting every $\mathcal{H}$-orbit and such that $\bar{V} \subset U \cap U^{\prime}$. By Lemma 4.3, there also exist $R, R^{\prime}>0$ such that any $d_{E}$-ball of radius $R$ in any $\mathcal{G}$-orbit meets $V$, and any $d_{E^{\prime}}$-ball of radius $R^{\prime}$ in any $\mathcal{G}^{\prime}$-orbit meets $V$. That is, for every $\mathcal{G}$-orbit $\mathcal{O}$ and for every $\mathcal{G}^{\prime}$-orbit $\mathcal{O}^{\prime}$, the intersection $\mathcal{O} \cap V$ is an $R$-net in $\mathcal{O}$ and $\mathcal{O}^{\prime} \cap V$ is an $R^{\prime}$-net in $\mathcal{O}^{\prime}$. So the result follows from Lemma 4.7

Proof of Theorem 4.6. Let $\Phi: \mathcal{H} \rightarrow \mathcal{H}^{\prime}$ be an equivalence, and let $\mathcal{H}^{\prime \prime}$ be the pseudogroup of local transformations of $Z^{\prime \prime}=Z \sqcup Z^{\prime}$ generated by $\mathcal{H} \cup \mathcal{H}^{\prime} \cup \Phi$, considered as sets of local transformations of $Z^{\prime \prime}$ in the obvious way. Then $U, U^{\prime}$ and $U^{\prime \prime}=U \sqcup U^{\prime}$ are relatively compact open subsets of $Z^{\prime \prime}$ that meet all $\mathcal{H}^{\prime \prime}$-orbits. The restrictions of $\mathcal{H}^{\prime \prime}$ to $U$ and $U^{\prime}$ are $\mathcal{G}$ and $\mathcal{G}^{\prime}$, thus $E$ and $E^{\prime}$ are recurrent systems of compact generation of $\mathcal{H}^{\prime \prime}$ on $U$ and $U^{\prime}$, respectively. Let $\mathcal{G}^{\prime \prime}$ denote the restriction of $\mathcal{H}^{\prime \prime}$ to $U^{\prime \prime}$, and select a recurrent system $E^{\prime \prime}$ of compact generation of $\mathcal{H}^{\prime \prime}$ on $U^{\prime \prime}$. By Corollary 4.8 , the $\mathcal{G}^{\prime \prime}$-orbits with $d_{E^{\prime \prime}}$ are uniformly coarsely quasi-isometric to the corresponding $\mathcal{G}$-orbits with $d_{E}$, and also to the corresponding $\mathcal{G}^{\prime}$-orbits with $d_{E^{\prime}}$. 
Let $\mathcal{H}$ be a compactly generated pseudogroup of local transformations of a locally compact space $Z, U$ a relatively compact open subset of $Z$ that meets all $\mathcal{H}$-orbits, and $\mathcal{G}$ the restriction of $\mathcal{H}$ to $U$. By considering the identity map on the $\mathcal{G}$-orbits and inclusions of systems of compact generation on $U$, we get an inductive system of metric spaces. Note also that distances between points in the same $\mathcal{G}$-orbit do not increase by considering larger systems of compact generation. By Theorem 4.6 the corresponding "inductive system of coarse quasi-isometry types" has a limit, which is uniformly reached just when a recurrent system of compact generation is considered. The following consequence of Lemma 4.7 shows that it happens so with the corresponding "inductive system of Lipschitz types."

Corollary 4.9. With the above notation, let E be a recurrent symmetric system of compact generation of $\mathcal{H}$ on $U$. Any other symmetric system $E^{\prime}$ of compact generation of $\mathcal{H}$ on $U$ is recurrent if and only if there exists some $C>0$ such that

$$
\frac{1}{C} d_{E^{\prime}}(x, y) \leq d_{E}(x, y) \leq C d_{E^{\prime}}(x, y)
$$

for all $x, y \in U$ lying in the same $\mathcal{G}$-orbit.

Proof. Fix any open set $V$ that meets all $\mathcal{G}$-orbits and with $\bar{V} \subset U$.

Suppose that $E^{\prime}$ is recurrent. Then, by Lemma4.3 there is some $R>0$ such that $\mathcal{O} \cap V$ is an $R$-net in $\left(\mathcal{O}, d_{E^{\prime}}\right)$ for every $\mathcal{G}$-orbit $\mathcal{O}$. To show that 4.1 holds for some $C>0$, assume first that $E^{\prime} \subset E$. Hence the first inequality of 4.1) holds for any $C \geq 1$. Take arbitrary points $x, y \in U$ lying in the same $\mathcal{G}$-orbit. We can assume that $x \neq y$, otherwise (4.1) holds trivially for any $C>0$. There are points $x^{\prime}, y^{\prime} \in V$ with $d_{E^{\prime}}\left(x, x^{\prime}\right), d_{E^{\prime}}\left(y, y^{\prime}\right) \leq R$. So $d_{E}\left(x, x^{\prime}\right), d_{E}\left(y, y^{\prime}\right) \leq R$ as well because $E^{\prime} \subset E$. By Lemma 4.7 there is some $C^{\prime}>0$, independent of $x^{\prime}, y^{\prime}$, such that

$$
\frac{1}{C^{\prime}} d_{E^{\prime}}\left(x^{\prime}, y^{\prime}\right) \leq d_{E}\left(x^{\prime}, y^{\prime}\right) \leq C^{\prime} d_{E^{\prime}}\left(x^{\prime}, y^{\prime}\right) \text {. }
$$

Therefore

$$
\begin{aligned}
d_{E}(x, y) & \leq d_{E}\left(x, x^{\prime}\right)+d_{E}\left(x^{\prime}, y^{\prime}\right)+d_{E}\left(y, y^{\prime}\right) \\
& \leq d_{E}\left(x^{\prime}, y^{\prime}\right)+2 R \\
& \leq C^{\prime} d_{E^{\prime}}\left(x^{\prime}, y^{\prime}\right)+2 R \\
& \leq C^{\prime}\left(d_{E^{\prime}}\left(x^{\prime}, x\right)+d_{E^{\prime}}(x, y)+d_{E^{\prime}}\left(y, y^{\prime}\right)\right)+2 R \\
& \leq C^{\prime}\left(d_{E^{\prime}}(x, y)+2 R\right)+2 R \\
& \leq\left(C^{\prime}+2 C^{\prime} R+2 R\right) d_{E^{\prime}}(x, y)
\end{aligned}
$$

since $d_{E^{\prime}}(x, y) \geq 1$, yielding the second inequality of 4.1) with $C=C^{\prime}+2 C^{\prime} R+2 R$.

When $E^{\prime} \not \subset E$, the union $E^{\prime \prime}=E \cup E^{\prime}$ is a recurrent symmetric system of compact generation of $\mathcal{H}$ on $U$. We have shown that there are some $C_{1}, C_{2}>0$ such that

$$
\begin{aligned}
& \frac{1}{C_{1}} d_{E^{\prime \prime}}(x, y) \leq d_{E}(x, y) \leq C_{1} d_{E^{\prime \prime}}(x, y), \\
& \frac{1}{C_{2}} d_{E^{\prime \prime}}(x, y) \leq d_{E^{\prime}}(x, y) \leq C_{2} d_{E^{\prime \prime}}(x, y)
\end{aligned}
$$

for all $x, y \in U$ lying in the same $\mathcal{G}$-orbit, and therefore (4.1) holds with $C=C_{1} C_{2}$.

Now assume that (4.1) holds for some $C>0$ and all $x, y \in U$ lying in the same $\mathcal{G}$-orbit. By Lemma 4.3, there is some $R>0$ such that $\mathcal{O} \cap V$ is an $R$-net in $\left(\mathcal{O}, d_{E}\right)$ for every $\mathcal{G}$-orbit 
$\mathcal{O}$. Then it easily follows that $\mathcal{O} \cap V$ is an $C R$-net in $\left(\mathcal{O}, d_{E^{\prime}}\right)$ for every $\mathcal{G}$-orbit $\mathcal{O}$, and thus $E^{\prime}$ is recurrent by Lemma 4.3

\section{FöLNER ORBITS}

The Fölner condition will be used in the next section to distinguish coarse quasi-isometry types of orbits. The property that Fölner orbits give rise to invariant measures will be needed also. This was shown by S. Goodman and J. Plante [6] for pseudogroups acting on compact metric spaces, and is partially improved in this section by using recurrent compact generation instead of a compact space. For compact foliated spaces, it is well known that Fölner leaves induce invariant transverse probability measures. So recurrence can be useful to show that compactly generated pseudogroups behave like compact foliated spaces, which is in the spirit of a famous project of A. Haefliger [10].

Let $M$ be a metric space with metric $d$. A quasi-lattice $\Gamma$ of $M$ is a net of $M$ such that for every $r \geq 0$ there is some $K_{r} \geq 0$ such that $\#(\Gamma \cap B(x, r)) \leq K_{r}$ for every $x \in M$. Not every metric space has a quasi-lattice, but metric spaces with bounded complexity in a reasonable sense do; see e.g. [4]) for examples. The metric space $M$ is said to be of coarse bounded geometry if it has a quasi-lattice.

For any $r>0$, the $r$-boundary of each subset $S \subset M$ is the set

$$
\partial_{r} S=\{x \in S \mid d(x, S)<r \text { and } d(x, M \backslash S)<r\} .
$$

The notation $\partial_{r}^{M} S$ will be also used for $\partial_{r} S$. Then $M$ is called amenable [4] if it has a quasi-lattice $\Gamma$ and a sequence of finite subsets $S_{n} \subset \Gamma$ such that

$$
\lim _{n \rightarrow \infty} \frac{\# \partial_{r}^{\Gamma} S_{n}}{\# S_{n}}=0
$$

for each $r>0$. Such a sequence $S_{n}$ will be called a Fölner sequence in $\Gamma$. Since

$$
\partial_{r}^{\Gamma} S \backslash S \subset \bigcup_{x \in S \cap \partial_{r}^{\Gamma} S}(\Gamma \cap B(x, r))
$$

for every $S \subset \Gamma$, it follows that

$$
\#\left(\partial_{r} S \backslash S\right) \leq K_{r} \cdot \#\left(S \cap \partial_{r} S\right)
$$

if \#( $\Gamma \cap B(x, r)) \leq K_{r}$ for any $x \in \Gamma$. Therefore the amenability condition (5.1) is equivalent to

$$
\lim _{n \rightarrow \infty} \frac{\#\left(S_{n} \cap \partial_{r}^{\Gamma} S_{n}\right)}{\# S_{n}}=0
$$

for each $r>0$.

Theorem 5.1 (Block-Weinberger [4]). Let $M, M^{\prime}$ be uniformly close metric spaces of coarse bounded geometry. Then $M$ is amenable if and only if so is $M^{\prime}$.

This result was proved in [4] in the following way. First, the uniformly finite homology $H_{\bullet}^{\mathrm{uf}}(M)$ is introduced for any metric space $M$. Second, it is shown that, if two metric spaces $M, M^{\prime}$ are uniformly close, then $H_{\bullet}^{\mathrm{uf}}(M) \cong H_{\bullet}^{\mathrm{uf}}\left(M^{\prime}\right)$. Finally, it is shown that a metric space $M$ of coarse bounded geometry is amenable if and only if $H_{0}^{\mathrm{uf}}(M) \neq 0$, and Theorem 5.1 follows. In particular, amenability is a coarse quasi-isometry invariant for metric spaces of coarse bounded geometry, which can be also proved directly without too much difficulty.

The following lemma will be useful in the in the proof of the main result of this section. 
Lemma 5.2. Let $\Gamma$ be a quasi-lattice in some metric space, and let $A$ be a $C$-net in $\Gamma$ for some $C>1$. Fix any $K>0$ such that every ball of radius $C$ in $\Gamma$ has at most $K$ points. Then

$$
\# S \leq \#\left(S \cap \partial_{C}^{\Gamma} S\right)+K \cdot \#(A \cap S)
$$

for any $S \subset \Gamma$.

Proof. For every $x \in S$, there is some $a \in A$ so that $d(x, a)<C$, and thus $x \in S \cap \partial_{C}^{\Gamma} S$ if $a \notin S$. Therefore

$$
S \subset\left(S \cap \partial_{C}^{\Gamma} S\right) \cup \bigcup_{a \in A \cap S}(\Gamma \cap B(a, C)),
$$

yielding the inequality of the statement.

We are interested in the case of a metric space $M$ whose points are the vertices of some connected graph, and where the distance between two points is the minimum number of contiguous edges needed to join them. In this case, besides the amenability condition of [4], $M$ may be also Fölner in the usual graph sense, which is defined as follows. The boundary $\partial S$ of any $S \subset M$ is the set of points $x \in S$ such that there is some edge joining $x$ with some point in $M \backslash S$; i.e., $\partial S=S \cap \partial_{2} S$ with the notation of [4]. Then $M$ is Fölner (as a graph) when there is a sequence of finite subsets $S_{n} \subset M$ such that

$$
\lim _{n \rightarrow \infty} \frac{\# \partial S_{n}}{\# S_{n}}=0 \text {. }
$$

Note that $M$ is a quasi-lattice in itself just when there is a uniform upper bound $K$ on the number of edges that meet at every vertex; indeed, $\# B(x, r) \leq K^{r}$ for all $r \geq 0$ when there is such a $K$. In this case, since

$$
\partial_{r} S \subset \bigcup_{x \in \partial S} B(x, r)
$$

it follows that

$$
\# \partial_{r} S \leq K^{r} \cdot \# \partial S
$$

for any $r>0$. Hence, when there is such a uniform upper bound $K, M$ is amenable (as metric space) if and only if it is Fölner (in the graph sense).

Consider again a pseudogroup $\mathcal{H}$ of local transformations of a space $Z$ with the metric $d_{E}$ on the orbits induced by a finite symmetric set $E$ of generators of $\mathcal{H}$. Then we get a graph by introducing an edge between two points $x, y \in Z$ whenever there is some $g \in E$ with $g(x)=y$. Thus \#E is an upper bound for the number of edges that meet at every vertex. Observe that each orbit of $\mathcal{H}$ is given by the vertices of a connected component of this graph, and $d_{E}$ is the metric induced by this graph on its connected components. The following notation and terminology will be used in this setting:

- Let $\partial^{E} S$ denote the boundary of any finite subset $S$ of an orbit with respect to the graph structure induced by $E$;

- for $r>0$, let $\partial_{r}^{E} S$ denote the $r$-boundary of any finite subset $S$ of an orbit with respect to the metric $d_{E}$;

- a Fölner sequence of an orbit with the metric $d_{E}$ (or with the graph structure induced by $E$ ) will be called an E-Fölner sequence; and

- an orbit with an E-Fölner sequence will be called E-Fölner or E-amenable.

Theorem 5.3. Let $\mathcal{H}$ be a compactly generated pseudogroup of local transformations of a locally compact metric space $Z$, let $U$ be a relatively compact open subset of $Z$ that meets all $\mathcal{H}$-orbits, and let $\mathcal{G}$ be the restriction of $\mathcal{H}$ to $U$. Consider the metric on the $\mathcal{G}$-orbits induced by a recurrent symmetric system $E$ of compact generation of $\mathcal{H}$ on $U$. If some 
G-orbit is E-Fölner, then there is a non-trivial non-negative G-invariant Borel measure on $U$ of finite mass.

Proof. Let $S_{n}$ be an E-Fölner sequence of some orbit $\mathcal{O}$ of $\mathcal{G}$. As in [6], a measure $\mu$ is constructed on $U$ as a limit of averaging measures on the finite sets $S_{n}$. Let $C_{0}(U)$ be the Banach space of continuous functions $f: U \rightarrow \mathbb{R}$ that vanish at infinity, endowed with the supremum norm || || given by

$$
\|f\|=\sup _{x \in U}|f(x)|
$$

For each $n \in \mathbb{N}$, let $\mu_{n}: C_{0}(U) \rightarrow \mathbb{R}$ be defined by

$$
\mu_{n}(f)=\frac{1}{\# S_{n}} \sum_{x \in S_{n}} f(x)
$$

for $f \in C_{0}(U)$. Each $\mu_{n}$ is obviously linear and continuous; i.e., it is an element of the (algebraic-topological) dual space $C_{0}(U)^{\prime}$. Moreover it is easy to check that

$$
\left|\mu_{n}(f)\right| \leq\|f\|
$$

for all $n \in \mathbb{N}$ and $f \in C_{0}(U)$. Therefore, by the Banach-Alaoglu theorem, the set $\left\{\mu_{n} \mid n \in\right.$ $\mathbb{N}\}$ is relatively compact in $C_{0}(U)^{\prime}$ with the weak topology; i.e., the topology of pointwise convergence. Then, by passing to a subsequence if necessary, we can assume that the sequence $\mu_{n}$ converges pointwise to some $\mu$ in $C_{0}(U)^{\prime}$, which can be considered as a Borel measure of finite mass on $U$ by the Riesz representation theorem. This $\mu$ is non-negative since all the $\mu_{n}$ are probability measures. The $\mathcal{G}$-invariance of $\mu$ follows from the $E$-Fölner condition of the sequence $S_{n}$ since, as shown in [6],

$$
|\mu(f \circ g)-\mu(f)| \leq 2\|f\| \lim _{n} \frac{\# \partial^{E} S_{n}}{\# S_{n}}
$$

for all $g \in E$ and $f \in C_{0}(U)$ with $\operatorname{supp} f \subset \operatorname{im} g$.

Finally, we show that $\mu$ is not trivial. Take any open set $U^{\prime}$ that meets all $\mathcal{G}$-orbits and with $\overline{U^{\prime}} \subset U$, and consider any non-negative function $f \in C_{0}(U)$ with $f(x)=1$ for all $x \in U^{\prime}$. By Lemma4.3, there is some $C>0$ such that $\mathcal{O} \cap U^{\prime}$ is a $C$-net in $\mathcal{O}$ with $d_{E}$. Then

$$
\mu(f) \geq \lim _{n} \frac{\#\left(S_{n} \cap U^{\prime}\right)}{\# S_{n}} \geq(\# E)^{-C} \lim _{n} \frac{\# S_{n}-\#\left(S_{n} \cap \partial_{C}^{E} S_{n}\right)}{\# S_{n}}=(\# E)^{-C}>0
$$

by Lemma $\left[5.2\right.$ and since the sequence $S_{n}$ is $E$-Fölner.

Remarks. (i) In the proof of Theorem 5.3, the measure $\mu$ could be trivial if $E$ were not recurrent. This is different from the arguments of [6] because $U$ is not compact. For instance, for the pseudogroup on $\mathbb{R}$ generated by the translation $g(x)=x+1$, the sets $\{n, n+1, \ldots, 2 n\}(n \in \mathbb{N})$ form a $\left\{g, g^{-1}\right\}$-Fölner sequence in an orbit, and the limit of corresponding averaging measures is trivial.

(ii) The statement of Theorem 5.3 could be more general. As in [6], the definitions and arguments could be modified to remove the condition that all sets of the Fölner sequence lie in the same orbit: it would be enough to have what is called an averaging sequence in [6]. But our result is simpler and general enough for our purposes in the next section. 


\section{AN EXAMPLE OF NON-RECURRENT COMPACT GENERATION}

In this section, we give an example showing that there exist non-recurrent systems of compact generation, and that the coarse quasi-isometry type of the orbits may depend on the system of compact generation if recurrence is not considered.

Fix real numbers

$$
a<a^{\prime}<a^{\prime \prime}<b^{\prime \prime}<b^{\prime}<b,
$$

and choose homeomorphisms $\phi: \mathbb{R} \rightarrow(a, b)$ and $\tilde{g}_{1}: \mathbb{R} \rightarrow \mathbb{R}$ satisfying the following properties:

- $\phi(x)=x$ for all $x \in\left[a^{\prime \prime}, b^{\prime \prime}\right]$;

- $\phi(x)>x$ for all $x \in\left(-\infty, a^{\prime \prime}\right)$;

- $\phi(x)<x$ for all $x \in\left(b^{\prime \prime}, \infty\right)$;

- $\phi(a)=a^{\prime}$ and $\phi(b)=b^{\prime}$

- $\tilde{g}_{1}(x)=x$ for all $x \in(-\infty, a] \cup[b, \infty)$;

- $\tilde{g}_{1}(x)>x$ for all $x \in(a, b)$; and

- $\tilde{g}_{1}\left(a^{\prime \prime}\right)<b^{\prime \prime}$.

Let $\mathcal{H}$ be the pseudogroup of local transformations of $\mathbb{R}$ generated by $\phi$ and $\tilde{g}_{1}$. The bounded open interval $U=(a, b)$ meets all $\mathcal{H}$-orbits because $\phi(\mathbb{R})=U$, and let $\mathcal{G}$ denote the restriction of $\mathcal{H}$ to $U$.

Now define a map $\tilde{g}_{2}: \mathbb{R} \rightarrow \mathbb{R}$ by setting

$$
\tilde{g}_{2}(x)= \begin{cases}\phi \circ \tilde{g}_{1} \circ \phi^{-1}(x) & \text { if } a<x<b, \\ x & \text { otherwise. }\end{cases}
$$

Such a $\tilde{g}_{2}$ is a homeomorphism and satisfies the following properties:

- $\tilde{g}_{2}(x)=x$ for all $x \in\left(-\infty, a^{\prime}\right] \cup\left[b^{\prime}, \infty\right)$;

- $\tilde{g}_{2}(x)>x$ for all $x \in\left(a^{\prime}, b^{\prime}\right)$; and

- $\phi \circ \tilde{g}_{1}(x)=\tilde{g}_{2} \circ \phi(x)$ for all $x \in U$.

We now prove that $\mathcal{G}$ is generated by the restrictions $\tilde{g}_{1}, \tilde{g}_{2}: U \rightarrow U$, which will be denoted by $g_{1}, g_{2}$. It is enough to prove that the restriction $\phi: U \rightarrow \phi(U)$ is in the pseudogroup $\mathcal{G}^{\prime}$ generated by $g_{1}, g_{2}$. Since $\tilde{g}_{1}\left(a^{\prime \prime}\right)<b^{\prime \prime}$, the collection of sets $U_{n}=g_{1}^{n}\left(a^{\prime \prime}, b^{\prime \prime}\right)$, $n \in \mathbb{Z}$, is an open covering of $U$, and thus it suffices to prove that each restriction $\phi: U_{n} \rightarrow$ $\phi\left(U_{n}\right)$ is in $\mathcal{G}^{\prime}$. But $\phi$ is the identity on $\left(a^{\prime \prime}, b^{\prime \prime}\right)=g_{1}^{-n}\left(U_{n}\right)$, yielding

$$
g_{1}^{-n}=\phi \circ g_{1}^{-n}=g_{2}^{-n} \circ \phi
$$

on $U_{n}$. So $\phi=g_{2}^{n} \circ g_{1}^{-n}$ on $U_{n}$, which belongs to $\mathcal{G}^{\prime}$, as desired.

Since $\overline{\operatorname{dom} g_{i}} \subset \operatorname{dom} \tilde{g}_{i}$ for $i=1,2$, it follows that $E=\left\{g_{1}, g_{2}, g_{1}^{-1}, g_{2}^{-1}\right\}$ is a system of compact generation of $\mathcal{H}$ on $U$. For the open subset $V=\left(a^{\prime}, b\right) \subset U$, it will be shown that

$$
\lim _{x \rightarrow a} d_{E}(x, V \cap \mathcal{G}(x)) \rightarrow \infty .
$$

Since $V$ meets every $\mathcal{G}$-orbit, it follows from Lemma 4.3 and (6.1) that $E$ is not recurrent.

To prove (6.1), let

$$
v(x)=\min \left\{n \in \mathbb{N} \mid g_{1}^{n}(x) \in V\right\}
$$

for each $x \in U$. Clearly,

$$
x \in V \Longleftrightarrow v(x)=0, \quad x<y \Longrightarrow v(x) \geq v(y), \quad \lim _{x \rightarrow a} v(x)=\infty .
$$

Take any $x \in U$ and some $h \in \mathcal{G}$ with

$$
x \in \operatorname{dom} h, \quad h(x) \in V, \quad|h|_{E, x}=d_{E}(x, V \cap \mathcal{G}(x)) .
$$


For $n=|h|_{E, x}$, we have $h=g_{i_{n}}^{\varepsilon_{n}} \cdots \circ g_{i_{1}}^{\varepsilon_{1}}$ around $x$ for some $i_{1}, \ldots, i_{n} \in\{1,2\}$ and $\varepsilon_{1}, \ldots, \varepsilon_{n} \in$ $\{ \pm 1\}$. Let $x_{k}=g_{i_{k}}^{\varepsilon_{k}} \circ \cdots \circ g_{i_{1}}^{\varepsilon_{1}}(x)$ for every $k=1, \ldots, n$. Since $x_{k} \notin V$ for each $k<n$, either $x_{k+1} \leq x_{k}$ (yielding $v\left(x_{k+1}\right) \geq v\left(x_{k}\right)$ ), or $g_{i_{k+1}}^{\varepsilon_{k+1}}=g_{1}$ (yielding $\left.v\left(x_{k+1}\right)=v\left(x_{k}\right)-1\right)$. Therefore

$$
v(x) \leq n=d_{E}(x, V \cap \mathcal{G}(x))
$$

because $v\left(x_{n}\right)=0$, and (6.1) follows.

Finally, let $F$ be a recurrent symmetric system of compact generation of $\mathcal{H}$ on $U$. We will show that no $\mathcal{G}$-orbit with the metric $d_{E}$ is coarsely quasi-isometric to itself with the metric $d_{F}$. Suppose that this is not true for some $\mathcal{G}$-orbit $\mathcal{O}$. Since the open interval $I=$ $\left(a, a^{\prime}\right)$ meets every orbit and since $g_{2}$ is the identity on $I$, there is some point $x_{0} \in \mathcal{O} \cap I$ such that the set

$$
X=\left\{g_{1}^{-m}\left(x_{0}\right) \mid m \in \mathbb{N}\right\} \subset \mathcal{O} \cap I
$$

satisfies $\partial^{E} X=\left\{x_{0}\right\}$. Hence $\mathcal{O}$ is $E$-Fölner; for instance, an $E$-Fölner sequence for $\mathcal{O}$ is given by the sets

$$
S_{n}=\left\{g_{1}^{-m}\left(x_{0}\right) \mid 0 \leq m \leq n\right\} .
$$

Then $\mathcal{O}$ is also $F$-Fölner by Theorem 5.1 since we are assuming that the metrics $d_{E}, d_{F}$ on $\mathcal{O}$ are coarsely quasi-isometric. So, by Theorem 5.3 and because $F$ is recurrent, there is a non-trivial non-negative $\mathcal{G}$-invariant Borel measure $\mu$ on $U$ of finite mass. Fix any $t \in U$, and let $I_{n}=\left(a, g_{1}^{n}(t)\right)$ for each $n \in \mathbb{Z}$. Since $\mu$ is $\mathcal{G}$-invariant and $g\left(I_{n}\right)=I_{n+1}$, all sets $I_{n}$ have the same $\mu$-measure, which is finite since $\mu$ has finite mass. Then $\mu\left(I_{n+1} \backslash I_{n}\right)=0$ for all $n$, whence $\mu(U)=0$ because $U=\bigcup_{n}\left(I_{n+1} \backslash I_{n}\right)$. This is a contradiction because $\mu$ is non-trivial and non-negative.

\section{QUASI-LOCAL METRIC SPACES}

The concept of equicontinuity can be defined for pseudogroups of local transformations of uniform spaces, but we are mainly concerned with the case of metric spaces in this paper. Nevertheless, it is enough to consider only certain part of the local geometry of metric spaces, which is extracted in the following definition. Moreover it is easier to work with pseudogroups and their equivalences when all other geometric information is removed from metric spaces.

Definition 7.1. Let $\left\{\left(Z_{i}, d_{i}\right)\right\}_{i \in I}$ be a family of metric spaces such that $\left\{Z_{i}\right\}_{i \in I}$ is a covering of a set $Z$, each intersection $Z_{i} \cap Z_{j}$ is open in $\left(Z_{i}, d_{i}\right)$ and $\left(Z_{j}, d_{j}\right)$, and for all $\varepsilon>0$ there is some $\delta(\varepsilon)>0$ so that the following property holds: for all $i, j \in I$ and $z \in Z_{i} \cap Z_{j}$, there is some open neighborhood $U_{i, j, z}$ of $z$ in $Z_{i} \cap Z_{j}$ (with respect to the topology induced by $d_{i}$ and $d_{j}$ ) such that

$$
d_{i}(x, y)<\delta(\varepsilon) \Longrightarrow d_{j}(x, y)<\varepsilon
$$

for all $\varepsilon>0$ and all $x, y \in U_{i, j, z}$. Such a family will be called a cover of $Z$ by quasi-locally equal metric spaces. Two such families are called quasi-locally equal when their union also is a cover of $Z$ by quasi-locally equal metric spaces. This is an equivalence relation whose equivalence classes are called quasi-local metrics on $Z$. For each quasi-local metric $\mathbb{Q}$ on $Z$, the pair $(Z, Q)$ is called a quasi-local metric space.

Any quasi-local metric $\mathfrak{Q}$ on $Z$ induces a uniformity so that, for any $\left\{\left(Z_{i}, d_{i}\right)\right\}_{i \in I} \in \mathfrak{Q}$, the families

$$
\mathcal{U}_{r}=\left\{\bigcup_{i \in I, x \in Z_{i}} B_{i}(x, r) \mid x \in Z\right\}, \quad r>0,
$$


form a base of uniform covers, where $B_{i}(x, r)$ denotes the open ball in $\left(Z_{i}, d_{i}\right)$ of center $x$ and radius $r$. The open balls of all metric spaces $\left(Z_{i}, d_{i}\right)$ form a base of the corresponding topology on $Z$. Any topological concept or property of $(Z, \mathfrak{Q})$ refers to this underlying topology. Any quasi-local metric space $(Z, Q)$ is locally metrizable, and thus first countable and completely regular. If $(Z, Q)$ is Hausdorff and paracompact, then it is metrizable [25] and normal [30, Theorem 20.10], and every point finite open cover of $(Z, \mathfrak{Q})$ is shrinkable [30, Theorem 15.10]. Moreover $(Z, Q)$ is a locally compact Polish space if and only if it is Hausdorff, paracompact, separable and locally compact; this is the type of quasi-local metric spaces that will be mainly considered in this paper.

Remark. A quasi-local metric is a "local structure" in the sense that it is determined by its "restriction" to the sets of any open covering. This property is specially useful to deal with pseudogroup equivalences, and is not satisfied by general uniformities. This is another reason to consider quasi-local metric spaces instead of general uniform spaces.

If a quasi-local metric space $(Z, \mathfrak{Q})$ is paracompact, then there is some $\left\{\left(Z_{i}, d_{i}\right)\right\}_{i \in I} \in \mathbb{Q}$ so that the covering $\left\{Z_{i}\right\}_{i \in I}$ is locally finite. In this case, $\left\{\left(Z_{i}, d_{i}\right)\right\}_{i \in I}$ satisfies the following slightly stronger condition.

Lemma 7.2. Let $(Z, Q)$ be a quasi-local metric space. If $\left\{Z_{i}\right\}_{i \in I}$ is locally finite for some $\left\{\left(Z_{i}, d_{i}\right)\right\}_{i \in I} \in \mathbb{Q}$, then there is some open neighborhood $U_{x}$ of each $x \in Z$ and some assignment $\varepsilon \mapsto \delta(\varepsilon)$ such that $(7.1)$ holds for all $\varepsilon>0, i, j \in I$ and $y \in U_{x} \cap Z_{i} \cap Z_{j}$.

Proof. With the notation of Definition 7.1, the set

$$
U_{z}=\bigcap_{i, j \in I,} U_{z \in Z_{i} \cap Z_{j}} U_{i, j, z}
$$

is an open neighborhood of each $z \in Z$ and satisfies the stated property.

Example 7.3. Any metric $d$ on a set $Z$ induces a unique quasi-local metric $\mathbb{Q}$ on $Z$ so that $\{(Z, d)\} \in \mathbb{Q}$. It will be shown in Section 14 that every Hausdorff paracompact quasilocal metric space $(Z, \mathfrak{Q})$ is indeed induced by some metric $d$ on $Z$ (Theorem 15.1), but the information of $(Z, d)$ contained in $\mathbb{Q}$ is just what is relevant for our study of equicontinuous pseudogroups.

Example 7.4. For each $t>0$, consider the metric $d_{t}$ on $\mathbb{R}^{2}$ defined by

$$
d_{t}(x, y)=\sqrt{t\left(x_{1}-y_{1}\right)^{2}+\left(x_{2}-y_{2}\right)^{2}}
$$

for $x=\left(x_{1}, x_{2}\right)$ and $y=\left(y_{1}, y_{2}\right)$. Then, for $T \subset \mathbb{R}_{+}$, the family $\left\{\left(\mathbb{R}^{2}, d_{t}\right) \mid t \in T\right\}$ is a cover of $\mathbb{R}^{2}$ by quasi-locally equal metric spaces if and only if $T$ is relatively compact in $\mathbb{R}_{+}$. Hence there are no maximal covers by quasi-locally equal metric spaces in general.

\section{EQUiCONTINUOUS PSEUDOGROUPS}

This section develops the concept of equicontinuity for pseudogroups, as suggested by E. Ghys in [17, Appendix E]. To motivate our definitions, consider a group $G$ of homeomorphisms of a space $Z$. On the one hand, some uniformity is needed on $Z$ for the usual definition of equicontinuity of $G$ (see, e.g., A. Weil [29]). But, on the other hand, equicontinuity of $G$ does not imply that each map in $G$ is uniformly continuous; i.e., these homeomorphisms may not preserve the uniformity of $Z$. This gives a difficulty when trying to generalize equicontinuity to pseudogroups in a way compatible with pseudogroup equivalences. More precisely, let $\mathcal{H}, \mathcal{H}^{\prime}$ be pseudogroups on spaces $Z, Z^{\prime}$, and $\Phi: \mathcal{H} \rightarrow \mathcal{H}^{\prime}$ 
an equivalence. Suppose that $\mathcal{H}$ is equicontinuous in some reasonable way, which should use some uniformity on $Z$. Then one has to use $\Phi$ to construct a uniformity on $Z^{\prime}$ so that $\mathcal{H}^{\prime}$ is equicontinuous too. The following is a standard way to do this kind of construction: the uniformity of $Z$ must be restricted to domains of homeomorphisms in $\Phi$, which are used to define uniformities on the sets of some open covering of $Z^{\prime}$, and then these local uniformities must be combined to yield a uniformity on the whole of $Z^{\prime}$. Some conditions must be satisfied to achieve such a combination. First, we need some type of uniformity that is determined by its restriction to the sets of any open covering, which holds for quasilocal metrics as indicated in the remark of Definition 7.1 Secondly, these uniformities on open sets of $Z^{\prime}$ must be compatible on the overlaps, which means that the local transformations of $\mathcal{H}$ must preserve the uniformity of $Z$; i.e., they must be uniformly continuous! Therefore the type of equicontinuity needed for pseudogroups seems to be "equi-uniform continuity;" i.e., the transformations of a pseudogroup are not only required to be "simultaneously" continuous at every point, but also required to be "simultaneously" uniformly continuous. Moreover, surprisingly, there are some unsolved difficulties to show that reasonable definitions of "equicontinuity at every point" and "equi-uniform continuity" are equivalent for compactly generated pseudogroups. So we define equicontinuity for pseudogroups by requiring that the "transformations with small domain" are "simultaneously" uniformly continuous. Indeed, what may be understood as "transformations with small domain" gives rise to two versions of equicontinuity. The first one is weaker and looks more natural, but the second one fits our needs.

Definition 8.1. Let $(Z, \mathfrak{Q})$ be a quasi-local metric space. A pseudogroup $\mathcal{H}$ of local homeomorphisms of $(Z, \mathbb{Q})$ is called weakly equicontinuous if, for some $\left\{\left(Z_{i}, d_{i}\right)\right\}_{i \in I} \in \mathbb{Q}$ and every $\varepsilon>0$, there is some $\delta(\varepsilon)>0$ so that the following property holds: for every $h \in \mathcal{H}, i, j \in I$ and $z \in Z_{i} \cap h^{-1}\left(Z_{j} \cap \operatorname{im} h\right)$, there is some neighborhood $U_{h, i, j, z}$ of $z$ in $Z_{i} \cap h^{-1}\left(Z_{j} \cap \operatorname{im} h\right)$ such that

$$
d_{i}(x, y)<\delta(\varepsilon) \Longrightarrow d_{j}(h(x), h(y))<\varepsilon
$$

for all $\varepsilon>0$ and $x, y \in U_{h, i, j, z}$

A pseudogroup $\mathcal{H}$ acting on a space $Z$ will be called weakly equicontinuous when it is weakly equicontinuous with respect to some quasi-local metric inducing the topology of $Z$.

Remarks. (i) Note that weak equicontinuity is a local property on $(Z, \mathfrak{Q})$ to a large extent; the only global aspect is the assignment $\varepsilon \mapsto \delta(\varepsilon)$, which is valid for all possible $h, i, j, z$.

(ii) The condition (7.1) of Definition 7.1 is the particular case of (8.1) for $h$ equal to the identity map on $Z$. So the whole structure of quasi-local metrics is needed to define weakly equicontinuous pseudogroups.

(iii) The condition of weak equicontinuity given in Definition 8.1 can be described as certain compatibility of $\mathcal{H}$ with $\mathfrak{Q}: \mathcal{H}$ is equicontinuous on $(Z, \mathfrak{Q})$ if and only if $\mathfrak{Q}$ can be realized as a combination of $h^{*}\left(\mathfrak{Q}_{\mid \mathrm{im} h}\right)$ for $h$ running through $\mathcal{H}$, where the restrictions, pull-backs and combinations of quasi-local metrics are defined in an obvious way (when appropriate conditions are satisfied).

(iv) In Definition 8.1, the assignment $\varepsilon \mapsto \delta(\varepsilon)$ depends on $\left\{\left(Z_{i}, d_{i}\right)\right\}_{i \in I} \in \mathbb{Q}$, but this definition is of course independent of the choice of $\left\{\left(Z_{i}, d_{i}\right)\right\}_{i \in I} \in \mathbb{Q}$; i.e., any other choice of $\left\{\left(Z_{i}, d_{i}\right)\right\}_{i \in I}$ satisfies the definition with some other assignment $\varepsilon \mapsto \delta(\varepsilon)$. 
The following result shows that weak equicontinuity is a property of equivalence classes of pseudogroups. The definition was worded in precisely such way for this property to hold true; in fact, this is rather evident by the above remarks (i) and (iii).

Lemma 8.2. Let $\mathcal{H}, \mathcal{H}^{\prime}$ be equivalent pseudogroups. Then $\mathcal{H}$ is weakly equicontinuous if and only if $\mathcal{H}^{\prime}$ is equicontinuous.

Proof. Let $Z, Z^{\prime}$ be the acted on by $\mathcal{H}, \mathcal{H}^{\prime}$. Assuming that $\mathcal{H}$ is weakly equicontinuous with respect to some quasi-local metric $\mathfrak{Q}$ inducing the topology of $Z$, we will show that so is $\mathcal{H}^{\prime}$. Thus there is some $\left\{\left(Z_{i}, d_{i}\right)\right\}_{i \in I} \in \mathfrak{Q}$ and some assignment $\varepsilon \mapsto \delta(\varepsilon)$ such that, for all $h \in \mathcal{H}$ and $i, j \in I$, the condition (8.1) holds on some neighborhood $U_{h, i, j, z}$ of each $z \in Z_{i} \cap h^{-1}\left(Z_{j} \cap \operatorname{im} h\right)$.

Let $\Phi: \mathcal{H} \rightarrow \mathcal{H}^{\prime}$ be a pseudogroup equivalence. There is an open covering $\left\{Z_{a}^{\prime}\right\}_{a \in A}$ of $Z^{\prime}$ such that, for each $a \in A$, there is some $\phi_{a} \in \Phi_{0}$ and some $i_{a} \in I$ with $Z_{a}^{\prime} \subset \operatorname{im} \phi_{a}$ and $\operatorname{dom} \phi_{a} \subset Z_{i_{a}}$. Let $d_{a}^{\prime}$ denote the restriction to $Z_{a}^{\prime}$ of the metric on im $\phi_{a}$ that corresponds via $\phi_{a}$ to the restriction of $d_{i_{a}}$ to dom $\phi_{a}$. For

$$
h^{\prime} \in \mathcal{H}^{\prime}, \quad a, b \in A, \quad z^{\prime} \in Z_{a}^{\prime} \cap h^{\prime-1}\left(Z_{b}^{\prime} \cap \operatorname{im} h^{\prime}\right),
$$

let $U_{h^{\prime}, a, b, z^{\prime}}^{\prime}=\phi_{a}\left(U_{h, i_{a}, i_{b}, z}\right)$, where

$$
h=\phi_{b}^{-1} \circ h^{\prime} \circ \phi_{a} \in \mathcal{H}, \quad z=\phi_{a}^{-1}\left(z^{\prime}\right) \in Z_{i_{a}} \cap h^{-1}\left(Z_{i_{b}} \cap \operatorname{im} h\right) .
$$

Now, given any $\varepsilon>0$, suppose $d_{a}^{\prime}\left(x^{\prime}, y^{\prime}\right)<\delta(\varepsilon)$ for $x^{\prime}, y^{\prime} \in U_{h^{\prime}, a, b, z^{\prime}}^{\prime}$. Then the points $x=\phi_{a}^{-1}\left(x^{\prime}\right)$ and $y=\phi_{a}^{-1}\left(y^{\prime}\right)$ lie in $U_{h, i_{a}, i_{b}, z}$ and satisfy $d_{i_{a}}(x, y)<\delta$, yielding $d_{i_{b}}(h(x), h(y))<\varepsilon$ by (8.1), and thus $d_{b}^{\prime}\left(h^{\prime}\left(x^{\prime}\right), h^{\prime}\left(y^{\prime}\right)\right)<\varepsilon$. Therefore (8.1) is satisfied by $\mathcal{H}^{\prime},\left\{\left(Z_{a}^{\prime}, d_{a}^{\prime}\right)\right\}_{a \in A}$, the same assignment $\varepsilon \mapsto \delta(\varepsilon)$, and the above choice of neighborhoods $U_{h^{\prime}, a, b, z^{\prime}}^{\prime}$. It follows that $\left\{\left(Z_{a}^{\prime}, d_{a}^{\prime}\right)\right\}_{a \in A}$ is a cover of $Z^{\prime}$ by quasi-locally equal metric spaces (remark (ii) of Definition 8.1 , and $\mathcal{H}^{\prime}$ is weakly equicontinuous with respect to the corresponding quasi-local metric, which obviously induces the given topology of $Z^{\prime}$.

On paracompact spaces, the following slightly different description of weak equicontinuity will be useful to understand the stronger version.

Lemma 8.3. Let $\mathcal{H}$ a pseudogroups acting on a paracompact quasi-local metric space $(Z, \mathfrak{Q})$. Then $\mathcal{H}$ is weakly equicontinuous if and only if there is some $\left\{\left(Z_{i}, d_{i}\right)\right\}_{i \in I} \in \mathfrak{Q}$ and some symmetric subset $S \subset \mathcal{H}$ such that any germ of any map in $\mathcal{H}$ is the germ of some map in $S$ and, for every $\varepsilon>0$, there is some $\delta(\varepsilon)>0$ so that (8.1) holds for all $h \in S$, $i, j \in I$ and $x, y \in Z_{i} \cap h^{-1}\left(Z_{j} \cap \operatorname{im} h\right)$.

Proof. The "only if" part follows because, with the notation of Lemma 7.2 and Definition 8.1 if

$$
U_{h, z}=U_{z} \cap \bigcap_{i, j \in I, z \in Z_{i} \cap Z_{j}} U_{h, i, j, z}
$$

for $h \in \mathcal{H}, i, j \in I$ and $z \in \operatorname{dom} h$, then 8.1 is satisfied by the family $S$ of all possible restrictions $h: U_{h, z} \rightarrow h\left(U_{h, z}\right)$.

Reciprocally, for all $h, i, j, z$ as in Definition 8.1 take $U_{h, i, j, z}$ equal to some open neighborhood of $z$ in $Z_{i} \cap h^{-1}\left(Z_{j} \cap \operatorname{im} h\right)$ where $h$ is equal some map in $S$. Then (8.1) obviously holds for all $x, y \in U_{h, i, j, z}$.

The stronger version of equicontinuity is defined by requiring that there is a set $S$ as in Lemma 8.3 that is also closed under compositions, which is some kind of a non-local condition . 
Definition 8.4. Let $\mathcal{H}$ be a pseudogroup of local homeomorphisms of a quasi-local metric space $(Z, \mathfrak{Q})$. Then $\mathcal{H}$ is called strongly equicontinuous if there exists some $\left\{\left(Z_{i}, d_{i}\right)\right\}_{i \in I} \in \mathfrak{Q}$ and some symmetric set $S$ of generators of $\mathcal{H}$ that is closed under compositions such that, for every $\varepsilon>0$, there is some $\delta(\varepsilon)>0$ so that (8.1) holds for all $h \in S, i, j \in I$ and $x, y \in Z_{i} \cap h^{-1}\left(Z_{j} \cap \operatorname{im} h\right)$.

A pseudogroup $\mathcal{H}$ acting on a space $Z$ will be called strongly equicontinuous when it is strongly equicontinuous with respect to some quasi-local metric inducing the topology of $Z$.

Remarks. (i) A typical choice of $S$ in Definition 8.4 is the set of all possible composites of some symmetric set of generators. In fact, given any $S$ satisfying the condition of strong equicontinuity, it is obviously possible to find a symmetric set of generators $E$ given by restrictions of elements of $S$, and then the set of all composites of elements of $E$ also satisfies the condition of strong equicontinuity.

(ii) If $h \in \mathcal{H}$ satisfies (8.1) for all $i, j \in I$ and $x, y \in Z_{i} \cap h^{-1}\left(Z_{j} \cap \operatorname{im} h\right)$, then so does its restriction to any open set. Hence $S$ can be assumed to be also closed under restrictions to open sets. Nevertheless, Definition 8.4 is not satisfactory with $S=\mathcal{H}$ because then the basic test of Example 8.6 below is not satisfied (see Example 8.7).

(iii) The definition of strong equicontinuity is independent of the choice of $\left\{\left(Z_{i}, d_{i}\right)\right\}_{i \in I} \in \mathbb{Q}$. Hence it is possible to assume that $\left\{Z_{i}\right\}_{i \in I}$ locally finite in Definition 8.4 when $(Z, \mathbb{Q})$ is paracompact.

Example 8.5. The pseudogroup $\mathcal{H}$ generated by the identity map on any quasi-local metric space $(Z, \mathfrak{Q})$ is obviously weakly equicontinuous by the remark (ii) of Definition 8.1 If $(Z, \mathfrak{Q})$ is paracompact, then $\mathcal{H}$ is also strongly equicontinuous by Lemma 7.2 , in fact, the definition of strong equicontinuity is satisfied with $S$ equal to the family of the identity maps on all finite intersections of the sets $U_{z}$ given by Lemma 7.2

Example 8.6. Recall that a group $G$ of homeomorphisms of a metric space $(Z, d)$ is equicontinuous, or better "equi-uniformly continuous," if for every $\varepsilon>0$ there is some $\delta(\varepsilon)>0$ such that

$$
d(x, y)<\delta(\varepsilon) \Longrightarrow d(g(x), g(y))<\varepsilon
$$

for all $g \in G$ and $x, y \in Z$. The pseudogroup $\mathcal{H}$ generated by such a $G$ is strongly equicontinuous because Definition 8.4 is satisfied with $S=G$ and $\left\{\left(Z_{i}, d_{i}\right)\right\}_{i \in I}=\{(Z, d)\}$. Of course, if $(Z, d)$ is compact, $G$ is "equicontinuous at every point" if and only if it is "equi-uniformly continuous."

Example 8.7. The group of translations on $\mathbb{R}$ is strongly equicontinuous with respect to the euclidean metric, and thus generates a strongly equicontinuous pseudogroup $\mathcal{H}$ with respect to the corresponding quasi-local metric. The following simple argument shows that some proper subset $S \subset \mathcal{H}$ must be taken to verify the definition of strong equicontinuous. Let $\left\{Z_{i}\right\}_{i \in I}$ be any open covering of $\mathbb{R}$, and $d_{i}$ a metric on each $Z_{i}$ inducing its topology. For any fixed index $i$, take real numbers $a<b$ such that $[a, b] \subset Z_{i}$. Let $r>0$ so that $0<r<\frac{b-a}{3}$. Let

$$
U=(a, a+r) \cup(a+r, a+2 r), \quad V=(a, a+r) \cup(b-r, b),
$$

which are contained in $Z_{i}$, and let $h: U \rightarrow V$ be the map in $\mathcal{H}$ defined by

$$
h(x)= \begin{cases}x & \text { if } a<x<a+r, \\ x+b-a-2 r & \text { if } a+r<x<a+2 r .\end{cases}
$$


The points

$$
x_{n}=a+\frac{n-1}{n} r, \quad y_{n}=a+\frac{n+1}{n} r
$$

satisfy the following properties:

- $x_{n}, y_{n} \in U$;

- $d_{i}\left(x_{n}, y_{n}\right) \rightarrow 0$ because $x_{n}, y_{n} \rightarrow a$ in $Z_{i}$; and

- $d_{i}\left(h\left(x_{n}\right), h\left(y_{n}\right)\right)>C$ for some $C>0$ since $h\left(x_{n}\right) \rightarrow a$ and $h\left(y_{n}\right) \rightarrow b$ in $Z_{i}$.

Then $h$ does not satisfy $(8.1)$ for any $\left\{\left(Z_{i}, d_{i}\right)\right\}_{i \in I}$ as above.

Even though an apparently non-local condition was added to define strong equicontinuity, the following result shows that this property is invariant by equivalences of pseudogroups acting on locally compact Polish spaces.

Lemma 8.8. If $\mathcal{H}, \mathcal{H}^{\prime}$ are equivalent pseudogroups acting on locally compact Polish spaces. Then $\mathcal{H}$ is strongly equicontinuous if and only if $\mathcal{H}^{\prime}$ strongly equicontinuous.

Proof. Let $\Phi: \mathcal{H} \rightarrow \mathcal{H}^{\prime}$ be a pseudogroup equivalence. Let $Z, Z^{\prime}$ be the locally compact Polish spaces acted on by $\mathcal{H}, \mathcal{H}^{\prime}$. Assuming that $\mathcal{H}$ is strongly equicontinuous with respect to some quasi-local metric $\mathfrak{Q}$ inducing the topology of $Z$, we will show that so is $\mathcal{H}^{\prime}$. Thus $\mathcal{H}$ satisfies the condition of strong equicontinuity for some $\left\{\left(Z_{i}, d_{i}\right)\right\}_{i \in I} \in \mathbb{Q}$ and some symmetric set $S$ of generators of $\mathcal{H}$ that is closed under compositions. By the remark (ii) of Definition 8.4, we can assume that $S$ is also closed under restrictions to open sets; so every transformation of $\mathcal{H}$ is a combination of maps in $S$.

Claim 1. There is a subset $\Phi_{0} \subset \Phi$ such that:

- For each $\phi \in \Phi_{0}$, there is some $i \in I$ so that $\operatorname{dom} \phi \subset Z_{i}$;

- $Z^{\prime}=\bigcup_{\phi \in \Phi_{0}}$ im $\phi$; and

- $\phi^{-1} \circ \psi \in S$ for all $\phi, \psi \in \Phi_{0}$.

To prove Claim 11 first note that, since $Z^{\prime}$ is a locally compact Polish space and $\Phi$ an equivalence, there is a sequence $\phi_{1}, \phi_{2}, \ldots$ in $\Phi$ such that:

- The domain of each $\phi_{n}$ is contained in some $Z_{i}$;

- $Z^{\prime}=\bigcup_{n} \operatorname{im} \phi_{n}$;

- the domain of each $\phi_{n}$ is relatively compact in $Z$; and

- every $\phi_{n}$ is a restriction of some $\tilde{\phi}_{n} \in \Phi$ with $\overline{\operatorname{dom} \phi_{n}} \subset \operatorname{dom} \tilde{\phi}_{n}$.

Then an increasing sequence of finite subsets $\Phi_{0, n} \subset \Phi$ is defined by induction on $n$ so that $\phi^{-1} \circ \psi \in S$ for all $\phi, \psi \in \Phi_{0, n}$ and

$$
\operatorname{im} \phi_{1} \cup \cdots \cup \operatorname{im} \phi_{n} \subset \bigcup_{\phi \in \Phi_{0, n}} \operatorname{im} \phi .
$$

Let $\Phi_{0,0}=\emptyset$ to begin with, and assume that $\Phi_{0, n}$ is defined for some $n \geq 0$ and satisfies the stated properties. To define $\Phi_{0, n+1}$, first set $\Phi_{0, n}^{\prime}=\Phi_{0, n} \cup\left\{\tilde{\phi}_{n+1}\right\}$. There is an open covering $\mathcal{U}_{n+1}$ of $\operatorname{dom} \tilde{\phi}_{n+1}$ such that the restriction of $\phi^{-1} \circ \tilde{\phi}_{n+1}$ to every $U \in \mathcal{U}_{n+1}$ is in $S$ for all $\phi \in \Phi_{0, n}^{\prime}$, which follows since $\Phi$ is an equivalence, $\Phi_{0, n}^{\prime}$ is finite and every map in $\mathcal{H}$ is a combination of maps in $S$. Then the compact set $\overline{\operatorname{dom} \phi_{n+1}}$ is covered by a finite subfamily $\mathcal{U}_{n+1}^{\prime} \subset \mathcal{U}_{n+1}$, and let $\Phi_{0, n+1}$ be the union of $\Phi_{0, n}$ and the set of restrictions of $\tilde{\phi}_{n+1}$ to all sets of $\mathcal{U}_{n+1}^{\prime}$. We get by induction that $\phi^{-1} \circ \psi \in S$ for all $\phi, \psi \in \Phi_{0, n+1}$ because $S$ is symmetric, and

$$
\operatorname{im} \phi_{1} \cup \cdots \cup \operatorname{im} \phi_{n+1} \subset \bigcup_{\phi \in \Phi_{0, n}} \operatorname{im} \phi \cup \operatorname{im} \phi_{n+1} \subset \bigcup_{\phi \in \Phi_{0, n+1}} \operatorname{im} \phi .
$$


Therefore Claim 1 follows with $\Phi_{0}=\bigcup_{n} \Phi_{0, n}$.

Now, let $S^{\prime}$ be the family of all possible composites $\phi \circ h \circ \psi^{-1}$ for $h \in S$ and $\phi, \psi \in \Phi_{0}$, which is symmetric and generates $\mathcal{H}^{\prime}$. Moreover the following argument shows that $S^{\prime}$ is closed under compositions. Take arbitrary elements $h_{1}^{\prime}, h_{2}^{\prime} \in S^{\prime}$; thus $h_{1}^{\prime}=\phi_{1} \circ h_{1} \circ \psi_{1}^{-1}$ and $h_{2}^{\prime}=\phi_{2} \circ h_{2} \circ \psi_{2}^{-1}$ for $h_{1}, h_{2} \in S$ and $\phi_{1}, \psi_{1}, \phi_{2}, \psi_{2} \in \Phi_{0}$. Since $\psi_{1}^{-1} \circ \phi_{2} \in S$ by Claim 1 it follows that $h_{1} \circ \psi_{1}^{-1} \circ \phi_{2} \circ h_{2} \in S$ because $S$ is closed under compositions. So

$$
h_{1}^{\prime} \circ h_{2}^{\prime}=\phi_{1} \circ h_{1} \circ \psi_{1}^{-1} \circ \phi_{2} \circ h_{2} \circ \psi_{2}^{-1} \in S^{\prime} .
$$

According to Claim 1, take any open covering $\left\{Z_{a}^{\prime}\right\}_{a \in A}$ of $Z^{\prime}$ such that, for each $a \in A$, there is some $\phi_{a} \in \Phi_{0}$ and some $i_{a} \in I$ with $Z_{a}^{\prime} \subset \operatorname{im} \phi_{a}$ and $\operatorname{dom} \phi_{a} \subset Z_{i_{a}}$. Let $d_{a}^{\prime}$ denote the restriction to $Z_{a}^{\prime}$ of the metric on $\operatorname{im} \phi_{a}$ that corresponds via $\phi_{a}$ to the restriction of $d_{i_{a}}$ to dom $\phi_{a}$. Choose an assignment $\varepsilon \mapsto \delta(\varepsilon)>0$ so that $S$ and $\left\{\left(Z_{i}, d_{i}\right)\right\}_{i \in I}$ satisfy the condition of strong equicontinuity. Let $h^{\prime}$ be an arbitrary element of $S^{\prime}$, which is equal to some composite $\phi \circ h \circ \psi^{-1}$ for $h \in S$ and $\phi, \psi \in \Phi_{0}$. Let $a, b \in A$ and $x^{\prime}, y^{\prime} \in Z_{a}^{\prime} \cap \operatorname{dom} h^{\prime}$ with $h^{\prime}\left(x^{\prime}\right), h^{\prime}\left(y^{\prime}\right) \in Z_{b}^{\prime}$, and let $g=\phi_{b}^{-1} \circ h^{\prime} \circ \phi_{a}$. Since $\psi^{-1} \circ \phi_{a}$ and $\phi_{b} \circ \phi^{-1}$ are in $S$ by Claim 1 it follows that

$$
g=\phi_{b}^{-1} \circ \phi \circ h \circ \psi^{-1} \circ \phi_{a} \in S
$$

because $S$ is closed under compositions. The points $x=\phi_{a}^{-1}\left(x^{\prime}\right)$ and $y=\phi_{a}^{-1}\left(y^{\prime}\right)$ lie in $Z_{i_{a}} \cap \operatorname{dom} g$, and $g(x), g(y) \in Z_{i_{b}}$. Moreover, if $d_{a}^{\prime}\left(x^{\prime}, y^{\prime}\right)<\delta(\varepsilon)$, then $d_{i_{a}}(x, y)<$ $\delta(\varepsilon)$, yielding $d_{i_{b}}(g(x), g(y))<\varepsilon$ by (8.1), and thus $d_{b}^{\prime}\left(h^{\prime}\left(x^{\prime}\right), h^{\prime}\left(y^{\prime}\right)\right)<\varepsilon$. It follows that $\left\{\left(Z_{a}^{\prime}, d_{a}^{\prime}\right)\right\}_{a \in A}$ is a cover of $Z^{\prime}$ by quasi-locally equal metric spaces (remark (ii) of Definition 8.1), and $\mathcal{H}^{\prime}$ satisfies the condition of strong equicontinuity with $S^{\prime}$ and $\left\{\left(Z_{a}^{\prime}, d_{a}^{\prime}\right)\right\}_{a \in A}$. Hence $\mathcal{H}^{\prime}$ is strongly equicontinuity with respect to the quasi-local metric $\mathfrak{Q}^{\prime}$ represented by $\left\{\left(Z_{a}^{\prime}, d_{a}^{\prime}\right)\right\}_{a \in A}$, which obviously induces the given topology of $Z^{\prime}$.

Problem 1. Give mild conditions so that weak and strong equicontinuity are equivalent.

Problem 2. It is possible to give pseudogroup versions of weak and strong "equicontinuity at every point" as in Definitions 8.1 and 8.4 Are they equivalent to our versions of equicontinuity (in the uniform sense) for compactly generated pseudogroups?

For the purposes of this paper, the key property of strong equicontinuity is the following.

Proposition 8.9. Let $\mathcal{H}$ be a compactly generated and strongly equicontinuous pseudogroup acting on a locally compact Polish quasi-local metric space $(Z, \mathfrak{Q})$, and let $U$ be any relatively compact open subset of $(Z, \mathfrak{Q})$ that meets every $\mathcal{H}$-orbit. Suppose that $\left\{\left(Z_{i}, d_{i}\right)\right\}_{i \in I} \in \mathbb{Q}$ satisfies the condition of Definition 8.4. E is any system of compact generation of $\mathcal{H}$ on $U$, and $\bar{g}$ satisfies the condition of Definition 2.3 for each $g \in E$. Let $\left\{Z_{i}^{\prime}\right\}_{i \in I}$ be any shrinking of $\left\{Z_{i}\right\}_{i \in I}$. Then there is a finite family $\mathcal{V}$ of open subsets of $(Z, \mathbb{Q})$ whose union contains $U$ and such that, for any $V \in \mathcal{V}, x \in U \cap V$, and $h \in \mathcal{H}$ with $x \in \operatorname{dom} h$ and $h(x) \in U$, the domain of $\tilde{h}=\bar{g}_{n} \circ \cdots \circ \bar{g}_{1}$ contains $V$ for any expression $h=g_{n} \circ \cdots \circ g_{1}$ around $x$ with $g_{1}, \ldots, g_{n} \in E$, and moreover $V \subset Z_{i_{0}}^{\prime}$ and $\tilde{h}(V) \subset Z_{i_{1}}^{\prime}$ for some $i_{0}, i_{1} \in I$.

Proof. We can assume that $\left\{Z_{i}\right\}_{i \in I}$ is locally finite. Let $S$ be a symmetric set of generators of $\mathcal{H}$ that is closed under compositions and restrictions to open subsets so that the condition of strong equicontinuity is satisfied by $S$ and $\left\{\left(Z_{i}, d_{i}\right)\right\}_{i \in I}$ (Definition 8.4).

Observe that any system $E$ of compact generation of $\mathcal{H}$ on $U$ satisfies the statement of this result if so does some other system of compact generation of $\mathcal{H}$ on $U$ whose elements are restrictions of elements of $E$. Therefore it can be assumed that, for all $g_{1}, g_{2} \in E$, we have

$$
\operatorname{dom} g_{1} \cap \operatorname{im} g_{2} \neq \emptyset \Longrightarrow \operatorname{dom} \bar{g}_{1} \cup \operatorname{im} \bar{g}_{2} \subset Z_{i}^{\prime}
$$


for some $i, j \in I$; in particular, since $E$ is symmetric, for each $g \in E$ there exists some $i, j \in I$ such that

$$
\operatorname{dom} \bar{g} \subset Z_{i}^{\prime}, \quad \operatorname{im} \bar{g} \subset Z_{j}^{\prime} .
$$

By the same reason, we can also suppose that $\bar{g} \in S$ for all $g \in E$.

Since $U$ is relatively compact and $\left\{Z_{i}\right\}_{i \in I}$ is locally finite, $U$ meets only a finite number of the sets $Z_{i}$. Thus there exists $\varepsilon>0$ such that

$$
Z_{i}^{\prime} \cap \operatorname{dom} g \neq \emptyset \Longrightarrow d_{i}\left(Z_{i}^{\prime} \cap \operatorname{dom} g, Z_{i} \backslash \operatorname{dom} \bar{g}\right)>\varepsilon,
$$

for all $i \in I$ and all $g \in E$, because $Z_{i}^{\prime} \cap \operatorname{dom} g$ is a relatively compact subset of $Z_{i}$. Let $\delta=\delta(\varepsilon)>0$ satisfy the condition of strong equicontinuity for such an $\varepsilon$; it is no restriction to assume that $\delta<\varepsilon$.

Let $\mathcal{V}$ be a finite family of open subsets of $Z$ whose union contains $U$ and such that each $V \in \mathcal{V}$ is contained in some $Z_{i}^{\prime}$ and has $d_{i}$-diameter smaller than $\delta$. Fix any $V \in \mathcal{V}$, $x \in U \cap V$ and $h \in \mathcal{H}$ with $x \in \operatorname{dom} h$ and $h(x) \in U$. Since $E$ generates the restriction of $\mathcal{H}$ to $U$, there exist $g_{1}, \ldots, g_{n} \in E$ so that $h=g_{n} \circ \cdots \circ g_{1}$ in some neighborhood of $x$, and let $\tilde{h}=\bar{g}_{n} \circ \cdots \circ \bar{g}_{1}$. If $V \subset \operatorname{dom} \tilde{h}$, then $V \subset Z_{i_{0}}^{\prime}$ and $\tilde{h}(V) \subset Z_{i_{1}}^{\prime}$ for some $i_{0}, i_{1} \in I$ by (8.3). So it only remains to show that $V \subset \operatorname{dom} \tilde{h}$, which will be done by induction on $n$.

The result is true for $n=1$. Indeed, $V \subset Z_{i}^{\prime}$ for some $i$, and $d_{i}(x, y)<\delta<\varepsilon$ for all $y \in V$. Thus $V \subset \operatorname{dom} \bar{g}_{1}$ by (8.4).

For $n>1$, let $f=g_{n-1} \circ \cdots \circ g_{1}$, which is defined in some neighborhood of $x$. By the induction hypothesis, the domain of $\tilde{f}=\bar{g}_{n-1} \circ \cdots \circ \bar{g}_{1}$ contains $V$. Then

$$
\operatorname{dom} g_{n} \cap \operatorname{im} g_{n-1} \neq \emptyset \text {, }
$$

and thus

$$
\operatorname{dom} \bar{g}_{n} \cup \operatorname{im} \bar{g}_{n-1} \subset Z_{j}^{\prime}
$$

for some $j \in I$ by $(8.2)$. In particular,

$$
\operatorname{im} \tilde{f} \subset \operatorname{im} \bar{g}_{n-1} \subset Z_{j}^{\prime},
$$

yielding $d_{j}(\tilde{f}(x), \tilde{f}(y))<\varepsilon$ for all $y \in V$ by strong equicontinuity since $d_{i}(x, y)<\delta$ and $\tilde{f} \in S$. Therefore $\tilde{f}(y) \in \operatorname{dom} \bar{g}_{n}$ by (8.4) because $\tilde{f}(x)=f(x) \in Z_{j}^{\prime} \cap \operatorname{dom} g_{n}$; i.e., the domain of $\tilde{h}=\bar{g}_{n} \circ \tilde{f}$ contains $V$ as desired.

Remarks. (i) With the notation of Proposition 8.9 given any symmetric set $S$ of generators of $\mathcal{H}$ that is closed under compositions, we can choose $E$ with the extensions $\bar{g}$ in $S$. So $S$ contains all maps $\tilde{h}$ of the statement of Proposition 8.9

(ii) Note that, with the conditions of Proposition 8.9, the pseudogroup $\mathcal{H}$ is complete as defined by Haefliger in [9].

It makes sense to consider the generalization to complete strongly equicontinuous pseudogroups of known results for complete pseudogroups of local isometries of Riemannian manifolds [8], [9]. But, for simplicity, only compactly generated strongly equicontinuous pseudogroups will be considered in this paper.

\section{QUASI-EFFECTIVE PSEUDOGROUPS}

Recall the following property that is invariant by equivalences of pseudogroups; it is interesting for our results on strongly equicontinuous pseudogroups. 
Definition 9.1 (Haefliger [8]). A pseudogroup $\mathcal{H}$ of local transformations of a space $Z$ is called quasi-analytic when the following holds for every $h \in \mathcal{H}$ : if $x \in \operatorname{dom} h$ and $h$ is the identity on some open set whose closure contains $x$, then $h$ is the identity on a neighborhood of $x$.

Example 9.2. Pseudogroups of local isometries of Riemannian manifolds are quasi-analytic because every such local isometry with connected domain is determined by its differential at any point.

Example 9.3. Let $Z$ be the compact subspace of $\mathbb{R}^{3}$ that is the union of a horizontal 2dimensional euclidean disk centered at the origin with a compact segment of the vertical axis containing the origin. Consider the quasi-local metric on $Z$ induced by the restriction of the euclidean metric of $\mathbb{R}^{3}$. The space $Z$ is invariant by rotations around the vertical axis. The pseudogroup $\mathcal{H}$ generated by any such non-trivial rotation is strongly equicontinuous but not quasi-analytic.

If $\mathcal{H}$ is a quasi-analytic pseudogroup on a space $Z$, then every $h \in \mathcal{H}$ with connected domain is the identity on $\operatorname{dom} h$ if it is the identity on some non-trivial open set. Because of this, quasi-analyticity is interesting for our purposes when $Z$ is locally connected, which is a very strong condition. To remove it, a slightly different property is defined inspired by the terminology of group actions.

Definition 9.4. A pseudogroup $\mathcal{H}$ of local transformations of a space $Z$ is said to be quasieffective if it is generated by some symmetric set $S$ that is closed under compositions, and such that any transformation in $S$ is the identity on its domain if it is the identity on some non-empty open subset of its domain.

Remarks. (i) In Definition 9.4, the family $S$ can be assumed to be also closed under restrictions to open sets. So every map in $\mathcal{H}$ is a combination of maps in $S$ in this case.

(ii) If the pseudogroup $\mathcal{H}$ is strongly equicontinuous and quasi-effective, then $\mathcal{H}$ is generated by a symmetric subset $S$ that is closed under compositions and satisfies the conditions of both Definitions 8.4 and 9.4

The following result can be proved with arguments similar to those in the proof of Lemma 8.8

Lemma 9.5. If $\mathcal{H}, \mathcal{H}^{\prime}$ are equivalent pseudogroups acting on locally compact Polish spaces $Z, Z^{\prime}$, then $\mathcal{H}$ is quasi-effective if and only if so is $\mathcal{H}^{\prime}$.

Lemma 9.6. Any quasi-effective pseudogroup is quasi-analytic.

Proof. Let $\mathcal{H}$ be a quasi-effective pseudogroup of local transformations of a space $Z$. So $\mathcal{H}$ satisfies the condition of Definition 9.4 with some symmetric set $S$ that generates $\mathcal{H}$ and is closed under compositions and restrictions to open sets (remark (i) of Definition 9.4). Then $\mathcal{H}$ is obviously quasi-analytic because any $h \in \mathcal{H}$ is a combination of elements of $S$.

Example 9.7. Let $r_{n}, s_{n}$ be two sequences of real numbers satisfying $0<r_{n}<s_{n}$ and $r_{n}, s_{n} \downarrow 0$. For each $n \in \mathbb{Z}_{+}$, let $U_{n}$ denote the (multiplicative) group of $n$th roots of 1 in $\mathbb{C}$, and fix a generator $\alpha_{n}$ of each $U_{n}$. Then let $Z$ be the compact subspace of $\mathbb{R} \times \mathbb{C}$ that is the union of the origin and the subspaces $\left\{s_{n}\right\} \times r_{n} U_{n}, n \in \mathbb{Z}_{+}$. Let $\mathcal{H}$ be the pseudogroup on $Z$ generated by the homeomorphism $h: Z \rightarrow Z$ that fixes the origin and satisfies $h\left(s_{n}, z\right)=\left(s_{n}, \alpha_{n} z\right)$ for any $z \in r_{n} U_{n}$ and $n \in \mathbb{Z}_{+}$. Note that $h$ is an isometry with respect to the restriction of the metric on $\mathbb{R} \times \mathbb{C}$ induced by the norm defined by $\|(t, z)\|=\max \{|t|,|z|\}$. 
So $\mathcal{H}$ is strongly equicontinuous with respect to the corresponding quasi-local metric on $Z$. Moreover $\mathcal{H}$ is quasi-analytic because, on the one hand, the origin is the only non-isolated point of $Z$ and, on the other hand, if some power $h^{m}$ is the identity on some open set whose closure contains the origin, then $m=0$. But $\mathcal{H}$ is not quasi-effective, as follows easily by using that, for any neighborhood $U$ of the origin in $Z$, there is some $m \in \mathbb{Z}_{+}$such that $h^{m}$ fixes some point in $U$ different from the origin, which is an open subset.

The above example shows that being quasi-effective is a strictly stronger property than quasi-analyticity, even for equicontinuous pseudogroups. Nevertheless, the following result shows that both properties are equivalent when quasi-analyticity fits our needs.

Lemma 9.8. Let $\mathcal{H}$ be a compactly generated strongly equicontinuous pseudogroup on a locally connected and locally compact Polish space $Z$. Then $\mathcal{H}$ is quasi-effective if and only if it is quasi-analytic.

Proof. The "only if" part was shown in Lemma9.6

Now assume that $\mathcal{H}$ is quasi-analytic. Let $U$ be any relatively compact open subset of $Z$ that meets every $\mathcal{H}$-orbit, and let $\mathcal{G}$ denote the restriction of $\mathcal{H}$ to $U$. By Lemma 9.5 , it is enough to show that $\mathcal{G}$ is quasi-effective. Let $E$ be any system of compact generation of $\mathcal{H}$ on $U$, and let $\bar{g}$ be an extension of each $g \in E$ satisfying the condition of Definition 2.3. Take a family $\mathcal{V}$ of open subsets of $Z$ satisfying the statement of Proposition 8.9 for the above $U, E$ and extensions $\bar{g}$. Since $Z$ is locally connected, we can assume that all sets in $\mathcal{V}$ are connected. Let $S$ be the set of maps $h \in \mathcal{G}$ such that:

- $h$ is a restriction of some composite of elements of $E$; and

- the domain and range of $h$ are contained in elements of $\mathcal{V}$.

Such an $S$ generates $\mathcal{G}$, is symmetric, and is closed under compositions and restrictions to open sets. Suppose that some $h \in S$ is the identity on some non-trivial open subset of its domain. We have

$$
h=g_{n} \circ \cdots \circ g_{1}: O \rightarrow P,
$$

where $g_{1}, \ldots, g_{n} \in E$ and $O, P$ are open subsets of $U$ that are contained in elements of $\mathcal{V}$; say $O \subset V \in \mathcal{V}$. Then the domain of $\tilde{h}=\bar{g}_{n} \circ \cdots \circ \bar{g}_{1}$ contains $V$ by Proposition 8.9. Since $h$ is the identity on some non-trivial open subset of $O$, the germ of $\tilde{h}$ at some point of $V$ is equal to the germ of the identity. So $\tilde{h}$ is the identity on $V$ because $\mathcal{H}$ is quasi-analytic and $V$ is connected. Thus $h$ is the identity on $O$ and the result follows.

The following result combines strong equicontinuity and quasi-effectiveness.

Proposition 9.9. Let $\mathcal{H}$ be a compactly generated, strongly equicontinuous and quasieffective pseudogroup of local homeomorphisms of a locally compact Polish space Z. Suppose that the conditions of strong equicontinuity and quasi-effectiveness are satisfied with a symmetric set $S$ of generators of $\mathcal{H}$ that is closed under compositions (Definitions 8.4 and (9.4). Let $A, B$ be open subsets of $Z$ such that $\bar{A}$ is compact and contained in $B$. If $x$ and $y$ are close enough points in $Z$, then

$$
f(x) \in A \Longrightarrow f(y) \in B
$$

for all $f \in S$ whose domain contains $x$ and $y$.

Proof. Suppose that the condition of strong equicontinuity is satisfied with $S$, some quasilocal metric $\mathfrak{Q}$, some $\left\{\left(Z_{i}, d_{i}\right)\right\}_{i \in I} \in \mathbb{Q}$ such that $\left\{Z_{i}\right\}_{i \in I}$ is locally finite, and some assignment $\varepsilon \mapsto \delta(\varepsilon)$. Let $\left\{Z_{i}^{\prime}\right\}_{i \in I}$ be a shrinking of the open covering $\left\{Z_{i}\right\}_{i \in I}$. Since $A$ is relatively 
compact and $\left\{Z_{i}\right\}_{i \in I}$ locally finite, $\bar{A}$ only meets finitely many of the sets $Z_{i}$. Thus, because each $Z_{i}^{\prime} \cap A$ is relatively compact in $Z_{i}$, there exists some $\varepsilon>0$ such that

$$
Z_{i}^{\prime} \cap A \neq \emptyset \neq Z_{i} \backslash B \Longrightarrow d_{i}\left(Z_{i}^{\prime} \cap A, Z_{i} \backslash B\right)>\varepsilon
$$

for all $i \in I$.

Fix $x, y \in Z$. Since $\mathcal{H}$ is compactly generated, $A$ and $x$ are contained in some relatively compact open $U$ that meets all orbits. Let $\mathcal{V}$ be a finite family of open sets that covers $\bar{U}$ and satisfies the conditions of Proposition 8.9. If $x$ and $y$ are close enough, then both of these points lie in some set $V \in \mathcal{V}$. Furthermore $V \subset Z_{i}$ for some $i \in I$, and we have $d_{i}(x, y)<\delta(\varepsilon)$ if $x$ and $y$ are close enough.

Now take any $f \in S$ with $x, y \in \operatorname{dom} f$ and $f(x) \in A$. According to Proposition 8.9, there is some $f^{\prime} \in S$ whose domain contains $V$ and so that $f, f^{\prime}$ have the same germ at $x$; furthermore there is some $j \in I$ such that $f^{\prime}(V) \subset Z_{j}$. In particular, $f(x)=f^{\prime}(x) \in Z_{j}$. Since $y \in \operatorname{dom} f \cap \operatorname{dom} f^{\prime}$, we also get $f(y)=f^{\prime}(y) \in Z_{j}$ by quasi-effectiveness. Therefore $d_{j}(f(x), f(y))<\varepsilon$ by strong equicontinuity, and the result follows from 9.1 .

\section{COARSE QUASI-ISOMETRY TYPE OF ORBITS WITH TRIVIAL GROUPS OF GERMS}

To compare different orbits of pseudogroups, some connection between them is needed; so the following terminology will be used. A pseudogroup $\mathcal{H}$ acting on a space $Z$ is called transitive when some orbit is dense in $Z$, and it is called minimal if every orbit is dense. A non-trivial subset $Y \subset Z$ is called minimal if it is closed, $\mathcal{H}$-invariant, and every orbit in $Y$ is dense in $Y$; equivalently, if $Y$ is a minimal element of the family of all non-trivial $\mathcal{H}$-invariant closed subsets of $Z$.

Theorem 10.1. Let $\mathcal{H}$ be a compactly generated, strongly equicontinuous and quasieffective pseudogroup of local transformations of a locally compact Polish space Z. Assume the space of orbits $Z / \mathcal{H}$ is connected (for example, if $\mathcal{H}$ is transitive). Let $\mathcal{G}$ denote the restriction of $\mathcal{H}$ to some relatively compact open subset $U \subset Z$ that meets every orbit. Then, with respect to any recurrent system of compact generation of $\mathcal{H}$ on $U$, all $\mathcal{G}$-orbits with trivial group of germs are uniformly coarsely quasi-isometric to each other.

Proof. Let $E$ be a recurrent system of compact generation of $\mathcal{H}$ on $U$, and for each $g \in E$ let $\bar{g}$ denote its extension satisfying the conditions of Definition 2.3 According to Corollary 4.5 and Theorem 4.6, it may be assumed that $\bar{E}=\{\bar{g} \mid g \in E\}$ is also a recurrent system of compact generation on some relatively compact open subset $U^{\prime} \subset Z$ with $\bar{U} \subset U^{\prime}$. Let $\mathcal{G}^{\prime}$ denote the restriction of $\mathcal{H}$ to $U^{\prime}$. By considering restrictions of elements of $\bar{E}$ to open subsets of their domains, we can assume that $\bar{E} \subset S$ for some subset $S \subset \mathcal{H}$ satisfying the conditions of Definition 9.4. Take a family $V$ of open subsets of $Z$ satisfying the statement of Proposition 8.9 for the above $U, E$ and extensions $\bar{g}$.

Since $Z$ is a Polish space, the union of orbits with trivial group of germs is a dense subset of $Z$. Hence, because $Z / \mathcal{H}$ is connected, it is enough to establish coarse quasiisometries between the $\mathcal{G}$-orbits of points $x, y \in U$ that are close enough to each other and have trivial group of germs; moreover the corresponding coarse distortions must be independent of $x$ and $y$. Thus it can be assumed that $x$ and $y$ are in the same element $V \in \mathcal{V}$. Consider the map $\phi_{x, y}: \mathcal{G}(x) \rightarrow \mathcal{G}^{\prime}(y)$ given by $h(x) \mapsto \tilde{h}(y)$, where $h \in \mathcal{G}, \tilde{h} \in S$, $x \in \operatorname{dom} h, V \subset \operatorname{dom} \tilde{h}$, and both $h, \tilde{h}$ have the same germ at $x$. Here, the germ of $h$ at $x$ is determined by the value $h(x)$ because the group of germs at $x$ is trivial. There exists such an $\tilde{h}$ for any $h$ by Proposition 8.9 and since $\bar{E} \subset S$. Moreover $\tilde{h}$ is unique on $V$ because $\mathcal{H}$ satisfies the condition of Definition 9.4 with $S$. Note also that $\phi_{x, y}$ takes values in $\mathcal{G}^{\prime}(y)$ by Proposition 8.9. Therefore $\phi_{x, y}$ is well defined. 
Claim 2. $\phi_{x, y}: \mathcal{G}(x) \rightarrow \mathcal{G}^{\prime}(y)$ is injective.

To prove this claim, take $f_{1}, f_{2} \in S$ whose domains contain $V$. So $\phi_{x, y}\left(f_{1}(x)\right)=f_{1}(y)$ and $\phi_{x, y}\left(f_{2}(x)\right)=f_{2}(y)$. If $f_{1}(y)=f_{2}(y)$, then $f_{1}, f_{2}$ have the same germ at $y$ because the group of germs of $\mathcal{H}$ at $y$ is trivial. It follows that $f_{1}=f_{2}$ on $V$ because both of these maps are in $S$ and their domains contain $V$. Hence $h_{1}(x)=h_{2}(y)$ as desired.

Claim 3. We have

$$
d_{\bar{E}}\left(\phi_{x, y}\left(z_{1}\right), \phi_{x, y}\left(z_{2}\right)\right) \leq d_{E}\left(z_{1}, z_{2}\right)
$$

for all $z_{1}, z_{2} \in \mathcal{G}(x)$.

We now show this assertion. We have $z_{1}=f_{1}(x), z_{2}=f_{2}(x), \phi_{x, y}\left(z_{1}\right)=f_{1}(y)$ and $\phi_{x, y}\left(z_{2}\right)=f_{2}(y)$ for some $f_{1}, f_{2} \in S$ whose domains contain $V$. Suppose $d_{E}\left(z_{1}, z_{2}\right)=k \geq 0$ in the orbit $\mathcal{G}(x)$. This means that there is a minimal decomposition $h_{2} \circ h_{1}^{-1}=g_{k} \circ \cdots \circ g_{1}$ about $z_{1}$ with $g_{1}, \ldots, g_{k} \in E$. Hence $f_{2} \circ f_{1}^{-1}$ and $\bar{g}_{k} \circ \cdots \circ \bar{g}_{1}$ are equal on $f_{1}(V)$ because both of these maps are in $S$ and their domains contain $f_{1}(V)$. This yields $d_{\bar{E}}\left(f_{1}(y), f_{2}(y)\right) \leq k$ in $\mathcal{G}^{\prime}(y)$, which finishes the proof of Claim 3

Let $A$ be an open subset of $Z$ intersecting every $\mathcal{H}$-orbit and such that $\bar{A} \subset U$.

Claim 4. There is some $C>0$, independent of $x, y$, such that

$$
d_{E}\left(z_{1}, z_{2}\right) \leq C d_{\bar{E}}\left(\phi_{x, y}\left(z_{1}\right), \phi_{x, y}\left(z_{2}\right)\right)
$$

for all $z_{1}, z_{2} \in \mathcal{G}(x) \cap A$.

To prove this estimate, take any $z_{1}, z_{2} \in \mathcal{G}(x)$. Again, there are $f_{1}, f_{2} \in S$, whose domains contain $V$, such that $z_{1}=f_{1}(x), z_{2}=f_{2}(x), \phi_{x, y}\left(z_{1}\right)=f_{1}(y)$ and $\phi_{x, y}\left(z_{2}\right)=f_{2}(y)$. Suppose $d_{\bar{E}}\left(f_{1}(y), f_{2}(y)\right)=k$. Then there is a decomposition $f_{2} \circ f_{1}^{-1}=\bar{g}_{k} \circ \cdots \circ \bar{g}_{1}$ around $f_{1}(y)$ for some $g_{1}, \ldots, g_{k} \in E$. So $f_{2} \circ f_{1}^{-1}=\bar{g}_{k} \circ \cdots \circ \bar{g}_{1}$ on $f_{1}(V)$ because both of these maps are in $S$ and their domains contain $f_{1}(V)$. It follows that $d_{\bar{E}}\left(z_{1}, z_{2}\right) \leq k$. Hence $d_{E}\left(z_{1}, z_{2}\right) \leq C k$ for some $C>0$ independent of $x$ and $y$ by Lemma 4.7 since $z_{1}, z_{2} \in A$ and $E, \bar{E}$ are recurrent systems of compact generation on $U, U^{\prime}$.

Claim 5. If $x, y$ are close enough, then $\mathcal{G}^{\prime}(y) \cap A \subset \phi_{x, y}(\mathcal{G}(x))$.

By Proposition 9.9, if $x, y$ are close enough in $V$, then

$$
f(y) \in A \Longrightarrow f(x) \in U
$$

for all $f \in S$ whose domain contains $V$. Then $\mathcal{G}^{\prime}(y) \cap A=\mathcal{G}(y) \cap A$ and thus every point in $\mathcal{G}^{\prime}(y) \cap A$ can be written as $f(y)$, for some $f \in \mathcal{G}$ whose domain contains $y$. By Proposition 8.9, there exists $\tilde{f} \in \mathcal{H}$ whose domain contains $V$, and such that $f$ and $\tilde{f}$ have the same germ at $y$. By (10.1), from $\tilde{f}(y)=f(y) \in A$, it follows that $\tilde{f}(x) \in U$. Thus the restriction $h$ of $\tilde{f}$ to some neighborhood of $x$ is in $\mathcal{G}$, and so

$$
f(y)=\tilde{f}(y)=\phi_{x, y}(h(x)) \in \phi_{x, y}(\mathcal{G}(x)),
$$

which shows Claim 5

Since $\bar{E}$ is recurrent, Lemma 4.3 implies that there exist $R, R^{\prime}>0$ such that every $d_{E^{-}}$ ball of radius $R$ in any $\mathcal{G}$-orbit meets $A$, as well as every $d_{\bar{E}}$-ball of radius $R^{\prime}$ in any $\mathcal{G}^{\prime}$-orbit. Therefore $\phi_{x, y}(\mathcal{G}(x) \cap A)$ is an $\left(R+R^{\prime}\right)$-net in $\left(\mathcal{G}^{\prime}(y), d_{\bar{E}}\right)$ by Claims 3 and 5 Moreover

$$
\phi_{x, y}:\left(\mathcal{G}(x) \cap A, d_{E}\right) \rightarrow\left(\phi_{x, y}(\mathcal{G}(x) \cap A), d_{\bar{E}}\right)
$$


is a bi-Lipschitz map whose distortion is independent of $x, y$ by Claims 2,3 and 4 Hence $\left(\mathcal{G}(x), d_{E}\right)$ is coarsely quasi-isometric to $\left(\mathcal{G}^{\prime}(y), d_{\bar{E}}\right)$, where the coarse distortions are independent of the choices of $x, y$. The result now follows from Theorem 4.6 since $E$ and $\bar{E}$ are recurrent systems of compact generation of $\mathcal{H}$ on $U, U^{\prime}$.

Example 10.2. In the pseudogroup $\mathcal{H}$ on $Z$ of Example 9.3 , all orbits have trivial groups of germs, except the origin. Moreover $Z$ is locally compact and locally connected, and $Z / \mathcal{H}$ is connected because so is $Z$. But, if $\mathcal{H}$ is generated by an irrational rotation, the statement of Theorem 10.1 does not hold because $\mathcal{H}$ is not quasi-analytic. Indeed, there are two coarse quasi-isometry types of orbits with trivial group of germs: the orbits of the points in the vertical segment are trivial, and all other orbits are quasi-isometric to the integers.

An action of a group $\Gamma$ on a space will be called quasi-effective when it generates a quasi-effective pseudogroup. A quasi-effective action may not be effective, as shown by the following example.

Example 10.3. Let $Z$ be a finite discrete space with more than two elements, and let $\Gamma$ be the group of all permutations of $Z$. Then the canonical action of $\Gamma$ on $Z$ is not effective but it is quasi-effective: the condition of Definition 9.4 is satisfied with the set $S$ of maps $\{x\} \rightarrow\{y\}$ with $x, y \in Z$.

Corollary 10.4. Let $\Gamma$ be a finitely generated group acting quasi-effectively and equicontinuously on a compact separable metric space $Z$ with connected space of orbits (for example, if some orbit is dense). Then all orbits with trivial group of germs are uniformly coarsely quasi-isometric to each other.

\section{Minimality of the orbit closures}

For compactly generated pseudogroups of local isometries of a Riemannian manifold, the closures of the orbits are manifolds, and the restriction of the pseudogroup to each orbit closure is a minimal pseudogroup [17, Appendix D] — this is just a pseudogroup version of Molino's theory for Riemannian foliations. In the more general situation considered here, at least the minimality of the orbit closures holds true.

Theorem 11.1. Let $\mathcal{H}$ be a compactly generated strongly equicontinuous pseudogroup of local transformations of a locally compact Polish space Z. Then the closure of each orbit is a minimal set. In particular, such a pseudogroup is minimal if it is transitive.

Proof. It is required to show that if the orbit of a point $x \in Z$ approaches another point $y$, then the orbit of $y$ also approaches $x$. If $U$ is a relatively compact open subset of $Z$ that meets every $\mathcal{H}$-orbit, then it can be assumed that $x, y \in U$.

Let $\mathcal{V}$ be a finite family of open subsets of $Z$ whose union covers $U$ as in Proposition 8.9 Let $V, W \in \mathcal{V}$ be such that $x \in V$ and $y \in W$. If $h_{n} \in \mathcal{H}$ is a sequence such that $h_{n}(x) \rightarrow y$, then it can be assumed that $\operatorname{dom} h_{n}=V$ and $h_{n}(x) \in W$ for all $n$. Moreover, there are maps $f_{n} \in \mathcal{H}$ with $\operatorname{dom} f_{n}=W$, and such that $f_{n}$ and $h_{n}^{-1}$ have the same germ at $h_{n}(x)$ for all $n$. Strong equicontinuity of $\mathcal{H}$ then implies that $f_{n}(y) \rightarrow x$ as follows. Suppose that the condition of strong equicontinuity of $\mathcal{H}$ is satisfied for a locally finite covering $\left\{\left(Z_{i}, d_{i}\right)\right\}_{i \in I}$ of $Z$ by quasi-locally equal metric spaces, some symmetric set $S$ of generators of $\mathcal{H}$ that is closed under compositions, and some assignment $\varepsilon \mapsto \delta(\varepsilon)$ (Definition 8.4). It may be assumed that $V \subset Z_{i}$ and $W \subset Z_{j}$ for some $i, j \in I$, and that $h_{n}, f_{n} \in S$ for all $n$. Given $\varepsilon>0$, there exists an integer $N>0$ such that $d_{j}\left(h_{n}(x), y\right)<\delta(\varepsilon)$ for all $n \geq N$. Hence

$$
d_{i}\left(x, f_{n}(y)\right)=d_{i}\left(f_{n} \circ h_{n}(x), f_{n}(y)\right)<\varepsilon
$$


for all $n \geq N$ by strong equicontinuity.

Corollary 11.2. Let $\mathcal{H}$ be a compactly generated and strongly equicontinuous pseudogroup of local transformations of a locally compact Polish space $Z$. Then the orbit closures of $\mathcal{H}$ define a partition of $Z$.

\section{The CLOSURE OF A STRONGLY EQUiCONTINUOUS PSEUDOGROUP}

In the study of pseudogroups of local isometries of Riemannian manifolds, an important role is played by the closure of such a pseudogroup [9]. It is defined by using the space of 1-jets, which is not available in our more general setting. But the closure of our type of pseudogroups can be also defined by using the compact-open topology on the spaces of local transformations defined on small enough open subsets.

As usual, for spaces $Y, Z$, let $C(Y, Z)$ denote the set of continuous maps $Y \rightarrow Z$, which will be denoted by $C_{\mathrm{c}-\mathrm{o}}(Y, Z)$ when it is endowed with the compact-open topology. For open subspaces $O, P$ of a space $Z$, the space $C_{\mathrm{c}-\mathrm{o}}(O, P)$ will be considered as an open subspace of $C_{\mathrm{c}-\mathrm{o}}(O, Z)$ in the canonical way.

Theorem 12.1. Let $\mathcal{H}$ be a quasi-effective, compactly generated and strongly equicontinuous pseudogroup of local transformations of a locally compact Polish space Z. Let $S$ be a symmetric set of generators of $\mathcal{H}$ that is closed under compositions and restrictions to open subsets, and satisfies the conditions of strong equicontinuity and quasi-effectiveness (Definitions 8.4 and 9.4). Let $\widetilde{\mathcal{H}}$ be the set of maps $h$ between open subsets of $Z$ that satisfy the following property: for every $x \in \operatorname{dom} h$, there exists a neighborhood $O_{x}$ of $x$ in $\operatorname{dom} h$ so that $\left.h\right|_{O_{x}}$ is in the closure of $C\left(O_{x}, Z\right) \cap S$ in $C_{\mathrm{c}-\mathrm{o}}\left(O_{x}, Z\right)$. Then:

- $\widetilde{\mathcal{H}}$ is closed under composition, combination and restriction to open sets;

- every map in $\widetilde{\mathcal{H}}$ is a homeomorphism around every point of its domain;

- the maps of $\widetilde{\mathcal{H}}$ that are homeomorphisms form a pseudogroup $\overline{\mathcal{H}}$ that contains $\mathcal{H}$;

- $\overline{\mathcal{H}}$ is strongly equicontinuous;

- the orbits of $\overline{\mathcal{H}}$ are equal to the closures of the orbits of $\mathcal{H}$; and

- $\widetilde{\mathcal{H}}$ and $\overline{\mathcal{H}}$ are independent of the choice of $S$.

Proof. The family $\widetilde{\mathcal{H}}$ is obviously closed under combination of maps and restrictions to open sets. Moreover $\widetilde{\mathcal{H}}$ is closed under composition of maps because $Z$ is locally compact Hausdorff (see e.g. [19, p. 289, Exercise 4]).

Given any relatively compact open subset $U$ that meets all $\mathcal{H}$-orbits, by Proposition 8.9 its remark (i) and Proposition 9.9 there is some finite family $\mathcal{V}$ of open subsets of $Z$ and another relatively compact open set $U_{0}$ such that:

- $\bar{U}$ is covered by the family $\mathcal{V}$;

- any germ of any map in the restriction of $\mathcal{H}$ to $U$ is a germ of some map in $S$ whose domain belongs to $\mathcal{V}$; and

- $f(V) \subset U_{0}$ for any $V \in \mathcal{V}$ and $f \in S$ with $V \subset \operatorname{dom} f$ and $f(V) \cap U \neq \emptyset$. In particular, any $V \in \mathcal{V}$ is contained in $U_{0}$.

For any map $h: V \rightarrow W$ in $\widetilde{\mathcal{H}}$ and any $x \in V$, we will show that there is some neighborhood $O$ of $x$ in $V$ such that the restriction $h: O \rightarrow h(O)$ is a homeomorphism whose inverse is also in $\widetilde{\mathcal{H}}$. It can be assumed that there are some $U, U_{0}$ and $\mathcal{V}$ as above so that $V, W \in \mathcal{V}$. Furthermore we can suppose that $h$ is the limit in $C_{\mathrm{c}-\mathrm{o}}(V, Z)$ of some sequence of maps $h_{n} \in C(V, Z) \cap S$. Take any open neighborhood $V^{\prime}$ of $x$ with $\overline{V^{\prime}} \subset V$. Since $\overline{V^{\prime}}$ is compact, it follows that $h_{n}\left(\overline{V^{\prime}}\right) \subset W$ for $n$ large enough. 
The germ of each $h_{n}^{-1}$ at $h_{n}(x)$ is equal to the germ of some $f_{n} \in S$ whose domain is $W$. By quasi-effectiveness, each $f_{n}$ is equal to $h_{n}^{-1}$ on $h_{n}(V) \cap W$, which contains $h_{n}\left(V^{\prime}\right)$. Hence $V^{\prime} \subset \operatorname{im} f_{n}$, and $f_{n}^{-1}$ is equal to $h_{n}$ on $V^{\prime}$.

By strong equicontinuity, the set $C\left(W, U_{0}\right) \cap S$ is equicontinuous in the usual sense. So, by the Ascoli theorem and the compactness of $\overline{U_{0}}$, we can assume that $f_{n}$ is convergent to some map $f$ in $C_{\mathrm{c}-\mathrm{o}}(W, Z)$, which is in $\widetilde{\mathcal{H}}$.

We have that $h_{n}(x) \rightarrow y=h(x)$, yielding $f_{n}(y) \rightarrow x$ by strong equicontinuity as in the proof of Theorem 11.1. Therefore $f(y)=x \in V^{\prime}$, and there is some open neighborhood $W^{\prime}$ of $y$ with $\overline{W^{\prime}} \subset W$ and $f\left(\overline{W^{\prime}}\right) \subset V^{\prime}$. Since $\overline{W^{\prime}}$ is compact, we get $f_{n}\left(\overline{W^{\prime}}\right) \subset V^{\prime}$ for $n$ large enough. So $f_{n}^{-1}$ is equal to $h_{n}$ on $f_{n}\left(W^{\prime}\right)$, yielding that the composite $h_{n} \circ f_{n}$ is the identity on $W^{\prime}$ for $n$ large enough. It follows that $h \circ f$ is the identity on $W^{\prime}$ because $Z$ is locally compact Hausdorff. Similarly, for any open neighborhood $O$ of $x$ with $\bar{O} \subset V^{\prime}$ and $h(\bar{O}) \subset W^{\prime}$, we get that $f \circ h$ is the identity on $O$. So $h: O \rightarrow h(O)$ is a homeomorphism whose inverse is $f: h(O) \rightarrow O$, which is in $\widetilde{\mathcal{H}}$ as desired.

Now, from what was proved for $\widetilde{\mathcal{H}}$, it follows directly that $\overline{\mathcal{H}}$ is a pseudogroup. Moreover $\overline{\mathcal{H}}$ contains $\mathcal{H}$ because $\widetilde{\mathcal{H}}$ contains $S$ by definition.

We now show that $\overline{\mathcal{H}}$ is strongly equicontinuous. Suppose that $\mathcal{H}$ satisfies the condition of strong equicontinuity (Definition 8.4 with the above $S$, some quasi-local metric $\mathfrak{Q}$, some $\left\{\left(Z_{i}, d_{i}\right)\right\}_{i \in I} \in \mathbb{Q}$ with $\left\{Z_{i}\right\}_{i \in I}$ locally finite, and some assignment $\varepsilon \mapsto \delta(\varepsilon)$. Let $\bar{S}$ be the set of homeomorphisms that are in the union of the closures of $C(O, Z) \cap S$ in $C_{\text {c-o }}(O, Z)$ with $O$ running on the open sets of $Z$. By definition, every element of $\overline{\mathcal{H}}$ is a combination of maps in $\bar{S}$. Since $S$ is closed under restrictions to open sets, it easily follows that so is $\bar{S}$. The set $\bar{S}$ is also closed under compositions because so is $S$ and $Z$ is locally compact Hausdorff. Moreover $\bar{S}$ is symmetric since it is closed under restrictions to open sets and because $\overline{\mathcal{H}}$ is a pseudogroup whose elements are combinations of maps in $\bar{S}$. A typical " $\varepsilon / 3$-argument" will show that $\overline{\mathcal{H}}$ satisfies the strong equicontinuity condition with $\bar{S}$ and the above family $\left\{\left(Z_{i}, d_{i}\right)\right\}_{i \in I}$. Take any $h: O \rightarrow P$ in $\bar{S}, i, j \in I$ and $x, y \in$ $Z_{i} \cap h^{-1}\left(Z_{j} \cap \operatorname{im} h\right)$. Suppose that $d_{i}(x, y)<\delta(\varepsilon / 3)$ for some $\varepsilon>0$. Such an $h$ is the limit of some sequence of maps $h_{n} \in C(O, Z) \cap S$ in $C_{\mathrm{c}-\mathrm{o}}(O, Z)$. On the one hand, since the compactopen topology is equal to the topology of uniform convergence on compact sets, it follows that $d_{j}\left(h_{n}(x), h(x)\right)<\varepsilon / 3$ and $d_{j}\left(h_{n}(x), h(x)\right)<\varepsilon / 3$ for $n$ large enough. On the other hand, we have $d_{j}\left(h_{n}(x), h_{n}(y)\right)<\varepsilon / 3$ for all $n$ since $h_{n} \in S$. Therefore $d_{j}(h(x), h(y))<\varepsilon$ as desired by the triangle inequality.

We now show that the orbits of $\overline{\mathcal{H}}$ are equal to the orbit closures of $\mathcal{H}$. Given two points $x, y$ in the same orbit closure of $\mathcal{H}$, it has to be shown that $x, y$ are in the same orbit of $\overline{\mathcal{H}}$. There is a sequence $h_{n} \in \mathcal{H}$ with $h_{n}(x) \rightarrow y$. It can be assumed that $x, y \in U$ for some relatively compact open set $U$ that meets all $\mathcal{H}$-orbits. As above, by Proposition 8.9, its remark (i) and Proposition 9.9, we can suppose that $h_{n} \in S$, $\operatorname{dom} h_{n}=V$ and $h_{n}(V) \subset U_{0}$ for some fixed open set $V$ and some relatively compact open set $U_{0}$. Thus $h_{n}$ is a sequence in $C\left(V, U_{0}\right) \cap S$, which is an equicontinuous family of maps. Therefore we can assume that $h_{n}$ is convergent in $C_{\mathrm{c}-\mathrm{o}}(V, Z)$ by the Ascoli theorem, and let $h$ be its limit. Then $h(x)=y$ and $h \in \widetilde{\mathcal{H}}$ by definition. Thus $x, y$ are in the same orbit of $\overline{\mathcal{H}}$ because the restriction of $h$ to some open neighborhood of $x$ is in $\overline{\mathcal{H}}$.

Finally $\overline{\mathcal{H}}$ is independent of the choice of $S$ because it is the pseudogroup generated by the local transformations of $Z$ lying in the union of closures of $C(O, Z) \cap \mathcal{H}$ in $C_{\mathrm{c}-\mathrm{o}}(O, Z)$ 
with $O$ running on the open sets of $Z$. Obviously, $\overline{\mathcal{H}}$ is independent of $S$ if and only if $\widetilde{\mathcal{H}}$ is also.

Definition 12.2. Let $\mathcal{H}$ be a quasi-effective, compactly generated and strongly equicontinuous pseudogroup of local transformations of a locally compact Polish space $Z$. With the notation of Theorem 12.1 the pseudogroup $\overline{\mathcal{H}}$ is called the closure of $\mathcal{H}$.

As a direct consequence of Theorem 12.1 in the present general setting, the orbit closures satisfy the following property of manifolds.

Definition 12.3. A topological space is homogeneous if the pseudogroup of all local homeomorphisms has exactly one orbit.

Corollary 12.4. Let $\mathcal{H}$ be a quasi-effective, compactly generated and strongly equicontinuous pseudogroup of local transformations of a locally compact Polish space Z. Then the closure of each orbit is homogeneous.

\section{LOCAL METRIC SPACES}

Pseudogroups of local isometries make sense on metric spaces but, with more generality, this type of pseudogroup can be defined on local metric spaces, which are introduced as follows.

Definition 13.1. Two metrics on the same set are said to be locally equal when they induce the same topology and each point has a neighborhood where both metrics are equal. Let $\left\{\left(Z_{i}, d_{i}\right)\right\}_{i \in I}$ be a family of metric spaces such that $\left\{Z_{i}\right\}_{i \in I}$ is a covering of a set $Z$, each intersection $Z_{i} \cap Z_{j}$ is open in $\left(Z_{i}, d_{i}\right)$ and $\left(Z_{j}, d_{j}\right)$, and the metrics $d_{i}, d_{j}$ are locally equal on $Z_{i} \cap Z_{j}$ whenever this is a non-empty set. Such a family will be called a cover of $Z$ by locally equal metric spaces. Two such families are called locally equal when their union also is a cover of $Z$ by locally equal metric spaces. This is an equivalence relation whose equivalence classes are called local metrics on $Z$. For each local metric $\mathfrak{D}$ on $Z$, the pair $(Z, D)$ is called a local metric space.

Remarks. (i) Observe the analogy between the definitions of local metrics and quasi-local metrics: for every local metric $\mathfrak{D}$, there is a unique quasi-local metric $\mathfrak{Q}$ so that $\mathfrak{D} \subset \mathbb{Q}$. In particular, all topological properties of quasi-local metric spaces hold for local metric spaces.

(ii) In contrast with quasi-local metrics, local metrics can be also characterized as maximal covers of $Z$ by locally equal metric spaces; there always exist such maximal families.

(iii) The concept of local metric has the following sheaf theoretic description, which shows its naturality. Suppose that the set $Z$ is endowed with a topology a priori, even though this topology will be later determined by the local metric. Then, for each open subset $U \subset Z$, let $\mathcal{M}(U)$ denote the set of all metrics on $U$ that induce its topology. Such an $\mathcal{M}$ is a presheaf on $Z$ with the usual restriction of metrics, and a local metric on $Z$ is just a global section of the sheaf $\widetilde{\mathcal{M}}$ determined by $\mathcal{M}$. By Example 13.2 below, the presheaf $\mathcal{M}$ is a sheaf only in the uninteresting case where the only metrizable open sets contain just one point.

Example 13.2. If $Z$ is metrizable and contains at least two points $x, y$, then there are infinitely many metrics that are locally equal to any given metric $d$ inducing the topology of $Z$; for instance, all the metrics $d_{r}, 0<r<d(x, y)$, given by $d_{r}\left(z, z^{\prime}\right)=\min \left\{d\left(z, z^{\prime}\right), r\right\}$ for $z, z^{\prime} \in U$. 
Example 13.3. Let $B$ be any open disc in $\mathbb{R}^{2}, Z=\mathbb{R}^{2} \backslash \bar{B}$. Let $d$ denote the restriction of the euclidean distance of $\mathbb{R}^{2}$ to $Z$, and $d^{\prime}$ the distance map on $Z$ induced by the restriction of the Riemannian metric of $\mathbb{R}^{2}$. Also, let $[x, y]$ denote the segment that joins each pair of points $x, y \in Z$. We have $d(x, y)=d^{\prime}(x, y)$ if $[x, y] \cap B=\emptyset$, and $d(x, y)<d^{\prime}(x, y)$ otherwise. So both metrics $d, d^{\prime}$ are locally equal, and thus define the same local metric space $(Z, \mathfrak{D})$.

The proof of the following result is analogous to the proof of Lemma 7.2 .

Lemma 13.4. Let $(Z, D)$ be a local metric space. If $\left\{Z_{i}\right\}_{i \in I}$ is locally finite for some $\left\{\left(Z_{i}, d_{i}\right)\right\}_{i \in I} \in \mathfrak{D}$, then there is some open neighborhood $U_{z}$ of each $z \in Z$ such that the metrics $d_{i}, d_{j}$ are equal on $U_{z} \cap Z_{i} \cap Z_{j}$ for all $i, j \in I$.

Each metric $d$ on a set $Z$ induces a unique local metric $\mathfrak{D}$ so that $\{(Z, d)\} \in \mathfrak{D}$. The following shows that the reciprocal holds when $(Z, \mathfrak{D})$ is Hausdorff and paracompact.

Theorem 13.5. A local metric space $(Z, D)$ is induced by some metric on $Z$ if and only if $(Z, \mathfrak{D})$ is Hausdorff and paracompact.

Proof. The "only if" part holds by the Stone theorem (see e.g. [30, Theorem 20.9]). Now suppose that $(Z, \mathfrak{D})$ is Hausdorff and paracompact.

Claim 6. There is some $\left\{\left(U_{a}, D_{a}\right)\right\}_{a \in A} \in \mathfrak{D}$ such that $\left\{U_{a}\right\}_{a \in A}$ is locally finite, and $D_{a}, D_{b}$ are equal on $U_{a} \cap U_{b}$ for all $a, b \in A$ with $U_{a} \cap U_{b} \neq \emptyset$.

Indeed, since $(Z, \mathfrak{D})$ is paracompact, there is some $\left\{\left(Z_{i}, d_{i}\right)\right\}_{i \in I} \in \mathfrak{D}$ such that $\left\{Z_{i}\right\}_{i \in I}$ is locally finite. Let $\left\{Z_{i}^{\prime}\right\}_{i \in I}$ be a shrinking of $\left\{Z_{i}\right\}_{i \in I}$. For each $i \in I$ and $x \in Z_{i}^{\prime}$, let $V_{i, x}$ be an open neighborhood of $x$ that is contained in $Z_{i}^{\prime}$ and meets only a finite number of sets $Z_{j}$ for $j \in I$. Therefore, for any $y \in V_{i, x}$, there is some open neighborhood $W_{i, x, y}$ of $y$ in $V_{i, x}$ such that:

- If $y \notin \overline{Z_{j}^{\prime}}$ for some $j \in I$, then $W_{i, x, y} \cap Z_{j}^{\prime}=\emptyset$;

- if $y \in \overline{Z_{j}^{\prime}}$ for some $j \in I$, then $W_{i, x, y} \subset Z_{j}$ and the metrics $d_{i}, d_{j}$ are equal on $W_{i, x, y}$.

Again, because $(Z, \mathfrak{D})$ is paracompact, there is an open locally finite refinement $\left\{U_{a}\right\}_{a \in A}$ of the open cover given by all possible sets $W_{i, x, y}$ as above. For each $a \in A$, choose any $W_{i, x, y}$ containing $U_{a}$, and let $D_{a}$ denote the restriction of $d_{i}$ to $U_{a}$. Then Claim 6 follows easily with such a family $\left\{\left(U_{a}, D_{a}\right)\right\}_{a \in A}$.

A metric $D$ on $Z$ is now defined as follows. With the notation of Claim 6 let $\left\{U_{a}^{\prime}\right\}_{a \in A}$ be a shrinking of the open covering $\left\{U_{a}\right\}_{a \in A}$. A pair $\left(z_{1}, z_{2}\right) \in Z \times Z$ will be said to be admissible if there is some $a \in A$ such that $z_{1}, z_{2} \in U_{a}^{\prime}$, and moreover

$$
\left\{z_{1}, z_{2}\right\} \cap U_{b}^{\prime} \neq \emptyset \Longrightarrow\left\{z_{1}, z_{2}\right\} \subset U_{b}
$$

for all $b \in A$. For each $(x, y) \in Z \times Z$, let $S_{x, y}$ denote the set of all finite sequences $\left(z_{0}, \ldots, z_{n}\right)$ in $Z$, with arbitrary length $n \in \mathbb{Z}_{+}$, such that $z_{0}=x, z_{n}=y$, and $\left(z_{k-1}, z_{k}\right)$ is an admissible pair for every $k=1, \ldots, n$. Then set $D(x, y)=1$ if $S_{x, y}=\emptyset$, and let

$$
D(x, y)=\inf _{\left(z_{0}, \ldots, z_{n}\right) \in S_{x, y}} \sum_{k=1}^{n} D_{a_{k}}\left(z_{k-1}, z_{k}\right)
$$

if $S_{x, y} \neq \emptyset$, where $z_{k-1}, z_{k} \in U_{a_{k}}^{\prime}$ with $a_{k} \in A$ for each $k=1, \ldots, n$. This definition is independent of the choices of the indices $a_{k}$ by Claim 6 .

Claim 7. Let $a \in A, x \in U_{a}^{\prime}$ and $y \in Z$ with $S_{x, y} \neq \emptyset$. Then

$$
D(x, y) \geq \begin{cases}\min \left\{D_{a}(x, y), D_{a}\left(x, U_{a} \backslash U_{a}^{\prime}\right)\right\} & \text { if } y \in U_{a}^{\prime}, \\ D_{a}\left(x, U_{a} \backslash U_{a}^{\prime}\right) & \text { if } y \notin U_{a}^{\prime} .\end{cases}
$$


To prove this assertion, let $\left(z_{0}, \ldots, z_{n}\right) \in S_{x, y}$ and $a_{1}, \ldots, a_{k} \in A$ with $z_{k-1}, z_{k} \in U_{a_{k}}^{\prime}$ for $k=1, \ldots, n$. On the one hand, if $z_{0}, \ldots, z_{n} \in U_{a}^{\prime}$, we have

$$
\sum_{k=1}^{n} D_{a_{k}}\left(z_{k-1}, z_{k}\right)=\sum_{k=1}^{n} D_{a}\left(z_{k-1}, z_{k}\right) \geq D_{a}\left(z_{0}, z_{n}\right)=D_{a}(x, y)
$$

by Claim 6. On the other hand, suppose $\left\{z_{0}, \ldots, z_{n}\right\} \not \subset U_{a}^{\prime}$. Then $n \geq 1$, and let

$$
n_{0}=\min \left\{k \in\{1, \ldots, n\} \mid z_{k} \notin U_{a}^{\prime}\right\} .
$$

Since $z_{n_{0}-1} \in U_{a}^{\prime}$, we get $z_{n_{0}} \in U_{a}$ because $\left(z_{n_{0}-1}, z_{n_{0}}\right)$ is an admissible pair. So

$$
\sum_{k=1}^{n} D_{a_{k}}\left(z_{k-1}, z_{k}\right) \geq \sum_{k=1}^{n_{0}} D_{a_{k}}\left(z_{k-1}, z_{k}\right) \geq D_{a}\left(z_{0}, z_{n_{0}}\right) \geq D_{a}\left(x, U_{a} \backslash U_{a}^{\prime}\right)
$$

by Claim 6 , which completes the proof of Claim 7

The above $D$ is a pseudometric on $Z$ because the following holds for all $x, y, z \in Z$ :

$$
\begin{aligned}
& (x, x) \in S_{x, x}, \\
& \left(z_{0}, \ldots, z_{n}\right) \in S_{x, y} \Longrightarrow\left(z_{n}, \ldots, z_{0}\right) \in S_{y, x},
\end{aligned}
$$

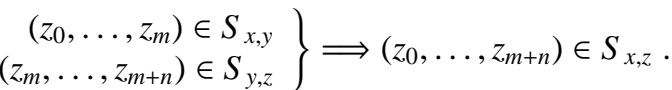

To show that $D$ is indeed a metric, suppose $D(x, y)=0$ for some $x, y \in Z$; thus $S_{x, y} \neq \emptyset$. Take any $a \in A$ with $x \in U_{a}^{\prime}$. Since $D_{a}\left(x, U_{a} \backslash U_{a}^{\prime}\right)>0$, it follows from Claim 7 that $y \in U_{a}^{\prime}$ and $D_{a}(x, y) \leq D(x, y)=0$. So $x=y$ as desired because $D_{a}$ is a metric.

It remains to check that $\{(Z, D)\} \in \mathcal{D}$. Fix any $z \in Z$ and any $a_{0} \in A$ with $z \in U_{a_{0}}^{\prime}$. The following assertion follows easily because $\left\{U_{a}\right\}_{a \in A}$ is locally finite and $\left\{U_{a}^{\prime}\right\}_{a \in A}$ is a shrinking of $\left\{U_{a}\right\}_{a \in A}$.

Claim 8. There is some open neighborhood $P_{z}$ of $z$ in $U_{a_{0}}^{\prime}$ such that

$$
P_{z} \cap U_{a}^{\prime} \neq \emptyset \Longrightarrow P_{z} \subset U_{a}
$$

for all $a, b \in A$.

Since $\left\{U_{a}\right\}_{a \in A}$ is locally finite and $(Z, D)$ is Hausdorff, the set

$$
O_{x}=\bigcap_{a \in A, x \in U_{a}^{\prime}} U_{a} \backslash \bigcup_{b \in A, x \notin U_{b}} \overline{U_{b}^{\prime}}
$$

is an open neighborhood of every $x$ in $Z$. If $x \in U_{a_{0}}^{\prime}$, it is easy to see that $(x, y) \in S_{x, y}$ for any $y \in U_{a_{0}}^{\prime} \cap O_{x}$, and thus $D(x, y) \leq D_{a_{0}}(x, y)$. Since

$$
x \in P_{z} \Longrightarrow P_{z} \subset O_{x}
$$

for all $x \in Z$ by Claim 8 it follows that $D(x, y) \leq D_{a_{0}}(x, y)$ for all $x, y \in P_{z}$.

On the other hand, we get from Claim 7 that $D(x, y) \geq D_{a_{0}}(x, y)$ for all $x \in U_{a_{0}}^{\prime}$ and all $y$ in the open ball in $\left(U_{a_{0}}, D_{a_{0}}\right)$ of center $x$ and radius $\rho(x)=D_{a_{0}}\left(x, U_{a_{0}} \backslash U_{a_{0}}^{\prime}\right)$. So $D(x, y) \geq$ $D_{a_{0}}(x, y)$ for all $x, y$ in the open ball in $\left(U_{a_{0}}, D_{a_{0}}\right)$ of center $z$ and radius $\frac{1}{2} \rho(z)$. Therefore the metrics $D, D_{a_{0}}$ are equal on some neighborhood of $x$, and the result follows.

Remarks. (i) Theorem 13.5] is very similar to the Smirnov metrization theorem [25], [19. pp. 260-261] (see also J. Nagata [20, Chapter VI.3] for a stronger result), which shows that a topological space is metrizable if and only if it is Hausdorff, paracompact and locally metrizable: in Theorem 13.5, the existence of a local metric is slightly stronger than local metrizability, and the existence of a metric that induces a given local metric is slightly stronger than metrizability. 
(ii) By the proof of Theorem 13.5 any paracompact Hausdorff local metric $\mathfrak{D}$ can be considered as the germ of some metric on $Z$ around the diagonal of $Z \times Z$. But even in this case, the introduction of local metrics makes sense to emphasize the fact that we are only considering distances between "very close" points.

(iii) With the sheaf theoretic point of view given in the remark (iii) of Definition 13.1, even though $\mathcal{M}$ is never a sheaf for interesting spaces, it is closer to be so for Hausdorff paracompact spaces: in this case, Theorem 13.5 asserts that the canonical homomorphism of presheaves, $\mathcal{M} \rightarrow \widetilde{\mathcal{M}}$, is surjective on all open sets.

Example 13.6. Let $P$ be the open upper half-plane $\left\{(x, y) \in \mathbb{R}^{2} \mid y>0\right\}$, and $L$ the real axis $\{(x, 0) \mid x \in \mathbb{R}\}$. Consider the half-disk topology on $Z=P \cup L$ [26, pp. 96-97], which has a base given by the euclidean open sets in $P$ and the sets of the form $\{z\} \cup(P \cap U)$, where $z \in L$ and $U$ is any euclidean open neighborhood of $z$ in $\mathbb{R}^{2}$. This space is not metrizable because it is not paracompact. But this topology is induced by a local metric $\mathfrak{D}$ on $Z$, which is determined by the family

$$
\left\{\left(P, d_{P}\right)\right\} \cup\left\{\left(U_{z}, d_{z}\right) \mid z \in L\right\},
$$

where $d_{P}$ is the restriction of the euclidean metric to $P, U_{z}=\{z\} \cup P$, and $d_{z}$ is the restriction of the euclidean metric to $U_{z}$.

Example 13.7. With more generality, let $(Z, d)$ be a metric space, let $\left\{Z_{i}\right\}_{i \in I}$ be a covering of $Z$, and let $d_{i}$ be the restriction of $d$ to $Z_{i}$ for each $i \in I$. Then the metrics $d_{i}, d_{j}$ have equal restriction to the overlap $Z_{i} \cap Z_{j}$ for all $i, j \in I$, and thus the family $\left\{\left(Z_{i}, d_{i}\right)\right\}_{i \in I}$ defines a local metric $\mathfrak{D}$ on $Z$. If the sets $Z_{i}$ are open in $(Z, d)$, then $\mathfrak{D}$ is induced by the metric $d$, otherwise the topology induced by $\mathfrak{D}$ is strictly finer than the topology induced by $d$, and $\mathcal{D}$ may not be induced by any metric, as in Example 13.6

Even though we are only interested on paracompact Hausdorff spaces, the following problem is interesting.

Problem 3. Is any locally metrizable topology induced by some local metric? In particular, is there a compatible local metric on every non-paracompact manifold? For instance, is there a compatible local metric on the Long Line [26. pp. 71-72]?

\section{Pseudogroups of Local isometries}

The idea of a local metric as measuring distances between "very close" points is specially appropriate to define local isometries.

Definition 14.1. Let $(Z, D)$ be a local metric space, and let $h$ be a homeomorphism between open subsets of $(Z, \mathfrak{D})$. Then $h$ is called a local isometry of $(Z, \mathfrak{D})$ if there is some $\left\{\left(Z_{i}, d_{i}\right)\right\}_{i \in I} \in \mathcal{D}$ such that, for $i, j \in I$ and $z \in Z_{i} \cap h^{-1}\left(Z_{j} \cap i m h\right)$, there is some neighborhood $U_{h, i, j, z}$ of $z$ in $Z_{i} \cap h^{-1}\left(Z_{j} \cap \operatorname{im} h\right)$ so that $d_{i}(x, y)=d_{j}(h(x), h(y))$ for all $x, y \in U_{h, i, j, z}$.

Remarks. (i) For a map $h$ between open subsets of a local metric space $(Z, \mathfrak{D})$, the property of being a local isometry is completely local, and $h$ may not be isometric for a given metric inducing $\mathfrak{D}$ (Examples 8.7 and 14.2).

(ii) About the condition that the metrics $d_{i}, d_{j}$ are locally equal on $Z_{i} \cap Z_{j}$ for any $\left\{\left(Z_{i}, d_{i}\right)\right\}_{i \in I} \in$ $\mathfrak{D}$, it just means that the identity map on any open subset of $(Z, \mathfrak{D})$ is a local isometry.

(iii) A homeomorphism $h$ between open subsets of a local metric space $(Z, \mathfrak{D})$ is a local isometry when it preserves the local metric in the obvious sense: $h^{*}\left(\mathfrak{D}_{\mid \operatorname{im} h}\right)=\mathfrak{D}_{\mid \operatorname{dom} h}$, where the restrictions and pull-backs of local metrics are defined in an obvious way. With the sheaf theoretic description of local metrics (remark (iii) of Definition 13.1), this means 
that $h$ induces an isomorphism between the restrictions of $\widetilde{\mathcal{M}}$ to its domain and image.

(iv) The definition of local isometry is completely independent of the choice of the family $\left\{\left(Z_{i}, d_{i}\right)\right\}_{i \in I} \in \mathcal{D}$. So the same $\left\{\left(Z_{i}, d_{i}\right)\right\}_{i \in I}$ can be chosen to verify Definition 14.1 for any family of local isometries. Therefore the concept of pseudogroup of local isometries is completely analogous to the concept of weakly equicontinuous pseudogroup.

Example 14.2. On the local metric space $(Z, \mathfrak{D})$ of Example 13.3 let $\mathcal{H}$ be the restriction of the pseudogroup generated by all translations of $\mathbb{R}^{2}$. Then $\mathcal{H}$ is a pseudogroup of local isometries of $(Z, \mathfrak{D})$. The maps in $\mathcal{H}$ with connected domain are isometries with respect to $d$, but many of them are not isometries with respect to $d^{\prime}$. For instance, let $U$ be any relatively compact and connected open subset of $Z$ containing points $x, y$ with $[x, y] \cap B \neq \emptyset$. Then there is a translation $h$ of $\mathbb{R}^{2}$ such that $h(U) \subset Z$ and $[h(x), h(y)] \cap B=\emptyset$. So

$$
d^{\prime}(h(x), h(y))=d(h(x), h(y))=d(x, y)<d^{\prime}(x, y),
$$

and thus the restriction $h: U \rightarrow h(U)$ is an element of $\mathcal{H}$ with connected domain that does not preserve $d^{\prime}$.

Arguments similar to those used in the proof of Lemma 8.2 prove the following lemma.

Lemma 14.3. Let $\mathcal{H}, \mathcal{H}^{\prime}$ be equivalent pseudogroups on spaces $Z, Z^{\prime}$. Then $\mathcal{H}$ is a pseudogroup of local isometries for some local metric inducing the topology of $Z$ if and only if $\mathcal{H}^{\prime}$ is a pseudogroup of local isometries for some local metric inducing the topology of $Z^{\prime}$.

Unlike the concept of equicontinuity, it is not necessary to introduce weak and strong versions of the concept of pseudogroup of local isometries by the following result.

Lemma 14.4. Let $\mathcal{H}$ be a pseudogroup of local transformations of a paracompact local metric space $(Z, \mathfrak{D})$. Then $\mathcal{H}$ is a pseudogroup of local isometries of $(Z, \mathfrak{D})$ if and only if there is some $\left\{\left(Z_{i}, d_{i}\right)\right\}_{i \in I} \in \mathfrak{D}$ and some symmetric set $S$ of generators of $\mathcal{H}$ that is closed under compositions and such that $d_{i}(x, y)=d_{j}(h(x), h(y))$ for all $h \in S, i, j \in I$ and $x, y \in Z_{i} \cap h^{-1}\left(Z_{j} \cap \operatorname{im} h\right)$.

Proof. Take any $\left\{\left(Z_{i}, d_{i}\right)\right\}_{i \in I} \in \mathfrak{D}$ such that $\left\{Z_{i}\right\}_{i \in I}$ is locally finite. With the notation of Lemma 13.4 and Definition 14.1, for each $h \in \mathcal{H}$ and $z \in \operatorname{dom} h$, let

$$
U_{h, z}=U_{z} \cap \bigcap_{i, j \in I, z \in Z_{i} \cap Z_{j}} U_{h, i, j, z},
$$

which is an open neighborhood of $z$. Then the result holds with $S$ equal to the set of compositions of all restrictions of the form $h: U_{h, z} \rightarrow h\left(U_{h, z}\right)$ and their inverses. We have used that composition of isometries is an isometry, which fails for the equicontinuous condition 8 . with a fixed assignment $\varepsilon \mapsto \delta(\varepsilon)$.

\section{ISOMETRIZATION OF STRONGLY EQUICONTINUOUS PSEUDOGROUPS}

On the type of spaces we are considering, it will be shown that compactly generated quasi-effective strongly equicontinuous pseudogroups are pseudogroups of local isometries for some local metric. We begin with the following version of Theorem 13.5 for quasi-local metric spaces. Most of its proof is also similar to the proof of Theorem 13.5, but there are some new difficulties.

Theorem 15.1. A quasi-local metric space $(Z, \mathfrak{Q})$ is induced by some metric on $Z$ if and only if $(Z, \mathfrak{Q})$ is Hausdorff and paracompact. 
Proof. As in the proof of Theorem 13.5 the "only if" part holds by the Stone theorem. Now suppose that $(Z, Q)$ is Hausdorff and paracompact. The following assertion can be proved in the same way as Claim 6 .

Claim 9. There is some $\left\{\left(U_{a}, D_{a}\right)\right\}_{a \in A} \in \mathbb{Q}$ and some $\delta(\varepsilon)>0$ for each $\varepsilon>0$ such that $\left\{U_{a}\right\}_{a \in A}$ is locally finite, and

$$
D_{a}(x, y)<\delta(\varepsilon) \Longrightarrow D_{b}(x, y)<\varepsilon
$$

for all $\varepsilon>0, a, b \in A$ and $x, y \in U_{a} \cap U_{b}$.

We can also assume that the family $\left\{\left(U_{a}, D_{a}\right)\right\}_{a \in A}$ given by Claim 6 satisfies that the $D_{a}$-diameter of each $U_{a}$ is smaller than 1 . If $x, y \in U_{a_{0}}$ for some $a_{0} \in A$, let

$$
\bar{D}(x, y)=\sup _{a \in A, x, y \in U_{a}} D_{a}(x, y) .
$$

Note that $\bar{D}(x, y) \leq 1$ by the condition on the $D_{a}$-diameter of each $U_{a}$, and that $\bar{D}(x, y)$ is independent of $a_{0}$. Moreover $\bar{D}$ is obviously symmetric, we have $\bar{D}(x, y)=0$ if and only if $x=y$, and the following assertion follows directly from Claim 9

Claim 10. We have

$$
D_{a}(x, y)<\delta(\varepsilon) \Longrightarrow \bar{D}(x, y)<\varepsilon
$$

for all $a \in A$ and $x, y \in U_{a}$.

But $\bar{D}$ may not satisfy the triangle inequality on any open set because there may be points $x, y, z \in U_{a_{0}}$ so that $x, y \in U_{a}$ and $z \notin U_{a}$ for some $a \in A$. So $\bar{D}$ may not be a metric on the sets of any open covering of $Z$; otherwise, Theorem 13.5 could be used to conclude. Yet $\bar{D}$ is used to define a metric on $Z$ with the idea of the proof of Theorem 13.5

Let $\left\{U_{a}^{\prime}\right\}_{a \in A}$ be a shrinking of $\left\{U_{a}\right\}_{a \in A}$. A pair $\left(z_{1}, z_{2}\right) \in Z \times Z$ will be said to be admissible if there is some $a \in A$ such that $z_{1}, z_{2} \in U_{a}^{\prime}$, and moreover

$$
\left\{z_{1}, z_{2}\right\} \cap U_{b}^{\prime} \neq \emptyset \Longrightarrow\left\{z_{1}, z_{2}\right\} \subset U_{b}
$$

for any $b \in A$. For each $(x, y) \in Z \times Z$, let $S_{x, y}$ denote the set of all finite sequences $\left(z_{0}, \ldots, z_{n}\right)$ in $Z$, with arbitrary length $n \in \mathbb{Z}_{+}$, such that $z_{0}=x, z_{n}=y$, and $\left(z_{k-1}, z_{k}\right)$ is an admissible pair for every $k=1, \ldots, n$. Now set $D(x, y)=1$ if $S_{x, y}=\emptyset$, and

$$
D(x, y)=\inf _{\left(z_{0}, \ldots, z_{n}\right) \in S_{x, y}} \sum_{k=1}^{n} \bar{D}\left(z_{k-1}, z_{k}\right)
$$

if $S_{x, y} \neq \emptyset$.

Claim 11. Let $a \in A, x \in U_{a}^{\prime}$ and $y \in Z$ with $S_{x, y} \neq \emptyset$. Then

$$
D(x, y) \geq \begin{cases}\min \left\{D_{a}(x, y), D_{a}\left(x, U_{a} \backslash U_{a}^{\prime}\right)\right\} & \text { if } y \in U_{a}^{\prime}, \\ D_{a}\left(x, U_{a} \backslash U_{a}^{\prime}\right) & \text { if } y \notin U_{a}^{\prime} .\end{cases}
$$

To prove this assertion, let $\left(z_{0}, \ldots, z_{n}\right) \in S_{x, y}$ and $a_{1}, \ldots, a_{k} \in A$ with $z_{k-1}, z_{k} \in U_{a_{k}}^{\prime}$ for $k=1, \ldots, n$. On the one hand, if $z_{0}, \ldots, z_{n} \in U_{a}^{\prime}$, we have

$$
\sum_{k=1}^{n} \bar{D}\left(z_{k-1}, z_{k}\right) \geq \sum_{k=1}^{n} D_{a}\left(z_{k-1}, z_{k}\right) \geq D_{a}\left(z_{0}, z_{n}\right)=D_{a}(x, y) .
$$

On the other hand, suppose $\left\{z_{0}, \ldots, z_{n}\right\} \not \subset U_{a}^{\prime}$. Then $n \geq 1$, and let

$$
n_{0}=\min \left\{k \in\{1, \ldots, n\} \mid z_{k} \notin U_{a}^{\prime}\right\} .
$$


Since $z_{n_{0}-1} \in U_{a}^{\prime}$, we get $z_{n_{0}} \in U_{a}$ because $\left(z_{n_{0}-1}, z_{n_{0}}\right)$ is an admissible pair. So

$$
\sum_{k=1}^{n} \bar{D}\left(z_{k-1}, z_{k}\right) \geq \sum_{k=1}^{n_{0}} D_{a}\left(z_{k-1}, z_{k}\right) \geq D_{a}\left(z_{0}, z_{n_{0}}\right) \geq D_{a}\left(x, U_{a} \backslash U_{a}^{\prime}\right),
$$

which completes the proof of Claim 7

With the same arguments as in the proof of Theorem 13.5 it follows that $D$ is a metric on $Z$ by using Claim 11

It remains to check that $\{(Z, D)\} \in \mathfrak{Q}$. Fix any $z \in Z$ and any $a_{0} \in A$ with $z \in U_{a_{0}}^{\prime}$. We get the following assertion as in the proof of Theorem 13.5

Claim 12. There is some open neighborhood $P_{z}$ of $z$ in $U_{a_{0}}^{\prime}$ such that

$$
P_{z} \cap U_{a}^{\prime} \neq \emptyset \Longrightarrow P_{z} \subset U_{a}
$$

for all $a, b \in A$.

Also, as in the proof of Theorem 13.5, the set

$$
O_{x}=\bigcap_{x \in U_{a}^{\prime}, a \in A} U_{a} \backslash \bigcup_{x \notin U_{b}, b \in A} \overline{U_{b}^{\prime}}
$$

is an open neighborhood of every $x$ in $Z$, and we have $(x, y) \in S_{x, y}$ for any $x \in U_{a_{0}}^{\prime}$ and $y \in U_{a_{0}}^{\prime} \cap O_{x}$. So $D(x, y) \leq \bar{D}(x, y)$ for all $y \in U_{a_{0}}^{\prime} \cap O_{x}$, yielding

$$
D_{a_{0}}(x, y)<\delta(\varepsilon) \Longrightarrow D(x, y)<\varepsilon
$$

by Claim 10, Since

$$
x \in P_{z} \Longrightarrow P_{z} \subset O_{x}
$$

for all $x \in Z$ by Claim 8 it follows that (15.1) holds for all $x, y \in P_{z}$.

On the other hand, as in the proof of Theorem 13.5, we get from Claim 11 that $D(x, y) \geq$ $D_{a_{0}}(x, y)$ for all $x, y$ in the open ball in $\left(U_{a_{0}}, D_{a_{0}}\right)$ of center $z$ and radius $\frac{1}{2} D_{a_{0}}\left(z, U_{a_{0}} \backslash U_{a_{0}}^{\prime}\right)$. Therefore the families of metric spaces $\{(Z, D)\}$ and $\left\{\left(U_{a}, D_{a}\right)\right\}_{a \in A}$ are quasi-locally equal; i.e., $\mathbb{Q}$ is induced by $D$.

Remarks. (i) Theorem 15.1 can be also compared with the Smirnov metrization theorem. (ii) By Theorem 15.1, in the paracompact Hausdorff case, a quasi-local metric is almost the same concept as a local metric; the only different being that different local metrics may induce the same quasi-local metric (Example 7.4).

Our "isometrization" result for pseudogroups can be stated as follows.

Theorem 15.2. Let $\mathcal{H}$ be a compactly generated, quasi-effective and strongly equicontinuous pseudogroup of local transformations of a locally compact Polish space Z. Then $\mathcal{H}$ is a pseudogroup of local isometries with respect to some local metric inducing the topology of $Z$.

Proof. The pseudogroup $\mathcal{H}$ is strongly equicontinuous with respect to some quasi-local metric $\mathfrak{Q}$ that induces the topology of $Z$. Such a $\mathbb{Q}$ is induced by some metric $d$ on $Z$ according to Theorem 15.1 So, by remark (iv) of Definition 8.4, the condition of strong equicontinuity is satisfied by the family $\{(Z, d)\}$ with some assignment $\varepsilon \mapsto \delta(\varepsilon)$ and some symmetric set $S$ of generators of $\mathcal{H}$ that is closed under compositions. We can also suppose that $S$ is closed under restrictions to open sets by remark (iii) of Definition 8.4 Furthermore we can assume that the condition of quasi-effectiveness is also satisfied with $S$ (remarks of Definition 9.4). This means that any element of $S$ is equal to the identity on its domain if 
it is equal to the identity on some non-trivial open subset; so two elements of $S$ are equal on the intersection of their domains if they have the same germ at some point.

Let $U$ be any relatively compact open subset of $Z$ that meets every $\mathcal{H}$-orbit, and $E$ any symmetric system of compact generation of $\mathcal{H}$ on $U$. For each $g \in E$, let $\bar{g}$ be its extension satisfying the conditions of Definition 2.3, and let $\bar{E}=\{\bar{g} \mid g \in E\}$. We can choose $S, E$ and the extensions $\bar{g}$ so that $\bar{E} \subset S$.

Let $V$ be a finite family of open subsets of $Z$ given by Proposition 8.9 for the above $d$, $S, U, E$ and extensions $\bar{g}$. We can suppose that the $d$-diameter of every $V \in V$ is smaller than $\delta(1)$. Let $R \subset \mathcal{H}$ be the set of all compositions of elements in $E$, and $\bar{R} \subset \mathcal{H}$ the set of all compositions of elements in $\bar{E}$; so $R, \bar{R} \subset S$. For each $V \in \mathcal{V}$, and $x, y \in V$, let

$$
d_{V}(x, y)=\sup _{h \in \bar{R}, V \subset \operatorname{dom} h} d(h(x), h(y)),
$$

Such $d_{V}$ is well defined by Proposition 8.9 , and we have $d_{V}(x, y) \leq 1$ by the condition on the diameter of $V$ and because $\bar{R} \subset S$. It is easy to check that $d_{V}$ is a metric on $V$. Moreover we have the following fact.

Claim 13. The metrics $d_{V}, d_{W}$ are equal on $V \cap W$ for all $V, W \in \mathcal{V}$.

Take sets $V, W \in \mathcal{W}$ with $V \cap W \neq \emptyset$ to verify this assertion. It suffices to show that, for all $h \in \bar{R}$ whose domain contains $V$, there is some $h^{\prime} \in \bar{R}$ whose domain contains $W$ and so that $h, h^{\prime}$ are equal on $V \cap W$ : this clearly yields $d_{V}(x, y) \leq d_{W}(x, y)$ for all $x, y \in V \cap W$, and the reverse inequality is similarly obtained. Thus let $h \in \bar{R}$ with $V \subset \operatorname{dom} h$. The germ of $h$ at any $x \in V \cap W$ is equal to the germ of some $f \in R$ at $x$. By Proposition 8.9, there is some $h^{\prime} \in \bar{R}$ whose domain contains $W$ and equal to $f$ around $x$. Since $h, h^{\prime} \in S$ and have the same germ at $x$, these maps are equal on $V \cap W$ by quasi-effectiveness, and the claim follows.

Therefore the collection $\left\{\left(V, d_{V}\right) \mid V \in \mathcal{V}\right\}$ defines a local metric $\mathfrak{D}_{0}$ on the union $U_{0}$ of the sets $V \in \mathcal{V}$. Moreover, on the one hand, we obviously have $d_{V}(x, y) \geq d(x, y)$ for all $V \in \mathcal{V}$ and $x, y \in V$. On the other hand,

$$
d(x, y)<\delta(\varepsilon) \Longrightarrow d_{V}(x, y)<\varepsilon
$$

for all $\varepsilon>0, V \in \mathcal{V}$ and $x, y \in V$ by strong equicontinuity since $\bar{R} \subset S$. Thus $\mathfrak{D}_{0}$ induces the restriction $\mathfrak{Q}_{0}$ of $\mathfrak{Q}$ to $U_{0}$.

Claim 14. We have

$$
d_{W}(f(x), f(y))=d_{V}(x, y)
$$

for all $V, W \in \mathcal{V}, f \in R$ and $x, y \in V \cap f^{-1}(W \cap \operatorname{im} h)$.

To prove this equality, let $V, W, f, x, y$ be as in the statement of this claim. Then we have $f=g_{m} \circ \cdots \circ g_{1}$ for $g_{1}, \ldots, g_{m} \in E$. Let $\tilde{f}=\bar{g}_{m} \circ \cdots \circ \bar{g}_{1} \in \bar{R}$. Then $V \subset \operatorname{dom} \tilde{f}$ by Proposition 8.9. For any $h \in \bar{R}$ with $W \subset \operatorname{dom} h$, the germ of $h$ at any fixed point $z \in W \cap \operatorname{im} f$ is equal to the germ at $z$ of some element of $R$; say $g_{m+n} \circ \cdots \circ g_{m+1}$ for some $g_{m+n}, \ldots, g_{m+1} \in E$. Hence $\bar{g}_{m+n} \circ \cdots \circ \bar{g}_{m+1} \in \bar{R}$ has the same germ at $z$ as $h$ and its domain contains $W$ again by Proposition 8.9. It follows that $h=\bar{g}_{m+n} \circ \cdots \circ \bar{g}_{m+1}$ on $W$ by quasi-effectiveness. Since $g_{m+n} \circ \cdots \circ g_{1}$ is defined around $f^{-1}(z) \in V$, the domain of $\bar{g}_{m+n} \circ \cdots \circ \bar{g}_{1}$ contains $V$ by Proposition 8.9 once more, and we have $h \circ \tilde{f}=\bar{g}_{m+n} \circ \cdots \circ \bar{g}_{1}$ on $V \cap \tilde{f}^{-1}(W \cap \operatorname{im} \tilde{f})$ by quasi-effectiveness. So $h \circ f$ is equal to some element of $\bar{R}$ on 
$V \cap f^{-1}(W \cap \operatorname{im} f)$, which yields

$$
\begin{aligned}
d_{W}(f(x), f(y)) & =\sup _{h \in \bar{R}, W \subset \operatorname{dom} h} d(h \circ f(x), h \circ f(y)) \\
& \leq \sup _{h^{\prime} \in \bar{R}, V \subset \operatorname{dom} h^{\prime}} d\left(h^{\prime}(x), h^{\prime}(y)\right) \\
& =d_{V}(x, y) .
\end{aligned}
$$

We also get $d_{W}(f(x), f(y)) \geq d_{V}(x, y)$ by applying the above argument to $f^{-1}$ instead of $f$, and Claim 14 follows.

Claim 14 shows that the restriction of $\mathcal{H}$ to $U$ is a pseudogroup of local isometries with respect to the restriction of $\mathfrak{D}_{0}$ to $U$, and therefore the theorem follows by Lemma 14.3 ,

According to Theorem 15.2, the following problem may be difficult and interesting.

Problem 4. Find an example of a strongly equicontinuous pseudogroup that is not a pseudogroup of local isometries for any local metric.

\section{A NON-STANDARD DESCRIPTION OF WEAK EQUICONTINUITY}

The following simple non-standard description of weak equicontinuity shows the naturality of this condition, even though strong equicontinuity is what is mainly used in our study. The reference for non-standard analysis is Robinson [21]; we do not use any technique particular to non-standard analysis, only the concept of monad, which is now defined.

Fix a non-principal ultrafilter $\mathcal{F}$ on the set $\mathbb{N}$ of positive integers; i.e., $\mathcal{F}$ defines a point in the corona of the Stone-Čech compactification of $\mathbb{N}$. Let $(Z, d)$ be any metric space. For any $x \in Z$, the monad of $x$ in $(Z, d)$, denoted by $M(x, Z, d)$ or simply $M(x)$, is the quotient set of the set of sequences $y_{n}$ in $Z$ such that

$$
\left\{n \in \mathbb{N} \mid d\left(x, y_{n}\right)<r\right\} \in \mathcal{F}
$$

for all $r>0$, where two such sequences $y_{n}, z_{n}$ are identified when

$$
\left\{n \in \mathbb{N} \mid y_{n}=z_{n}\right\} \in \mathcal{F} .
$$

If $\left(Z^{\prime}, d^{\prime}\right)$ is another metric space, any continuous map $f:(Z, d) \rightarrow\left(Z^{\prime}, d^{\prime}\right)$ induces a map $f_{*}: M(x, Z, d) \rightarrow M\left(f(x), Z^{\prime}, d^{\prime}\right)$ for every $x \in Z$, which is defined as follows: if $\mathbf{y} \in M(x, Z, d)$ is represented by the sequence $y_{n}$, then $f_{*}(\mathbf{y})$ is represented by the sequence $f\left(y_{n}\right)$.

The monad of 0 in $\mathbb{R}$ with the euclidean metric is the set $\mathbf{I}$ of infinitesimal numbers. The infinitesimal number represented by the zero constant sequence will be denoted by $\mathbf{0}$. For $\boldsymbol{\varepsilon}, \boldsymbol{\delta} \in \mathbf{I}$, represented by sequences $\varepsilon_{n}, \delta_{n}$, the inequality $\boldsymbol{\varepsilon}<\boldsymbol{\delta}$ means that

$$
\left\{n \in \mathbb{N} \mid \varepsilon_{n}<\delta_{n}\right\} \in \mathcal{F} .
$$

Moreover the metric $d$ on $Z$ defines a map $d_{*}: M(x) \rightarrow \mathbf{I}$ for every $x \in Z$ in the following way: if $\mathbf{y} \in M(x)$ is represented by the sequence $y_{n}$, then $d_{*}(\mathbf{y})$ is represented by the sequence $d\left(x, y_{n}\right)$.

Now suppose that $\mathfrak{Q}$ is a quasi-local metric on $Z$ and $\left\{\left(Z_{i}, d_{i}\right)\right\}_{i \in I} \in \mathfrak{Q}$. If $x \in Z_{i} \cap Z_{j}$ for $i, j \in I$, then $M\left(x, Z_{i}, d_{i}\right) \equiv M\left(x, Z_{j}, d_{j}\right)$ canonically. Thus $M\left(x, Z_{i}, d_{i}\right)$ can be called the monad of $x$ in $(Z, \mathfrak{Q})$, and denoted by $M(x, Z, \mathfrak{Q})$ or simply $M(x)$. It also follows that any continuous map between quasi-local metric spaces, $f:(Z, \mathfrak{Q}) \rightarrow\left(Z^{\prime}, \mathfrak{Q}^{\prime}\right)$, induces a map $f_{*}: M(x, Z, \mathfrak{Q}) \rightarrow M\left(f(x), Z^{\prime}, \mathfrak{Q}^{\prime}\right)$ for each $x \in Z$. 
Theorem 16.1. Let $\mathcal{H}$ be a pseudogroup of local homeomorphisms of a quasi-local metric space $(Z, \mathfrak{Q})$, and let $\left\{\left(Z_{i}, d_{i}\right)\right\}_{i \in I} \in \mathfrak{Q}$. Then $\mathcal{H}$ is weakly equicontinuous if and only if for every $\boldsymbol{\varepsilon} \in \mathbf{I}, \boldsymbol{\varepsilon}>\mathbf{0}$, there is some $\boldsymbol{\delta}(\boldsymbol{\varepsilon}) \in \mathbf{I}, \boldsymbol{\delta}(\boldsymbol{\varepsilon})>\mathbf{0}$, such that

$$
d_{i *}(\mathbf{y})<\delta(\varepsilon) \Longrightarrow d_{j *}\left(h_{*}(\mathbf{y})\right)<\varepsilon
$$

for all $h \in \mathcal{H}, i, j \in I, x \in Z_{i} \cap h^{-1}\left(Z_{j} \cap \operatorname{im} h\right)$ and $\mathbf{y} \in M(x)$.

Proof. Suppose first that $\mathcal{H}$ is weakly equicontinuous. So the condition of weak equicontinuity is satisfied with $\left\{\left(Z_{i}, d_{i}\right)\right\}_{i \in I}$, some assignment $\varepsilon \mapsto \delta(\varepsilon)$ and neighborhoods $U_{h, i, j, z}$ (Definition 8.1). We can assume that $\delta(\varepsilon)<\varepsilon$ for all $\varepsilon>0$. Given any $\boldsymbol{\varepsilon} \in \mathbf{I}, \boldsymbol{\varepsilon}>\mathbf{0}$, take some sequence $\varepsilon_{n}$ representing $\varepsilon$. We can assume that $\varepsilon_{n}>0$ for all $n$. Then the sequence $\delta\left(\varepsilon_{n}\right)$ also represents some infinitesimal number, which is denoted by $\delta(\varepsilon)$. Now take $h \in \mathcal{H}, i, j \in I, x \in Z_{i} \cap h^{-1}\left(Z_{j} \cap \operatorname{im} h\right)$ and $\mathbf{y} \in M(x)$ with $d_{i *}(\mathbf{y})<\delta(\boldsymbol{\varepsilon})$. So

$$
\left\{n \in \mathbb{N} \mid d_{i}\left(x, y_{n}\right)<\delta\left(\varepsilon_{n}\right)\right\} \in \mathcal{F} .
$$

Moreover

$$
\left\{n \in \mathbb{N} \mid y_{n} \in U_{h, i, j, x}\right\} \in \mathcal{F}
$$

because $\mathbf{y} \in M(x)$. Therefore

$$
\left\{n \in \mathbb{N} \mid y_{n} \in Z_{i} \cap h^{-1}\left(Z_{j} \cap \operatorname{im} h\right), d_{j}\left(h(x), h\left(y_{n}\right)\right)<\delta\left(\varepsilon_{n}\right)\right\} \in \mathcal{F}
$$

by weak equicontinuity, yielding $d_{j *}\left(h_{*}(\mathbf{y})\right)<\varepsilon$, and (16.1) follows.

Now suppose that (16.1) holds for some assignment $\boldsymbol{\varepsilon} \mapsto \delta(\varepsilon)$ and all $h, i, j, x, \mathbf{y}$ as in the statement. According to Definition 8.1 if $\mathcal{H}$ is not weakly equicontinuous, then there exists some $\varepsilon>0, h \in \mathcal{H}, i, j \in I$ and $z \in Z_{i} \cap h^{-1}\left(Z_{j} \cap i m h\right)$ so that, in every neighborhood $U$ of $z$ in $Z_{i} \cap h^{-1}\left(Z_{j} \cap\right.$ im $\left.h\right)$, there are points $x_{U}, y_{U}$ with $d_{j}\left(h\left(x_{U}\right), h\left(y_{U}\right)\right) \geq \varepsilon$. So, for every $n \in \mathcal{N}$, there are points $x_{n}, y_{n} \in Z_{i} \cap h^{-1}\left(Z_{j} \cap \operatorname{im} h\right)$ with

$$
d_{i}\left(x_{n}, z\right), d_{i}\left(y_{n}, z\right)<1 / n, \quad d_{j}\left(x_{n}, y_{n}\right) \geq \varepsilon .
$$

On the other hand, there is some $N \in \mathbb{N}$ so that $d_{j}\left(h(z), h\left(x_{n}\right)\right)<\varepsilon / 2$ for $n \geq N$. Thus

$$
d_{j}\left(h(z), h\left(y_{n}\right)\right) \geq d_{j}\left(h\left(x_{n}\right), h\left(y_{n}\right)\right)-d_{j}\left(h(z), h\left(x_{n}\right)\right)>\varepsilon / 2
$$

for $n \geq N$. Given any $\boldsymbol{\varepsilon} \in \mathbf{I}, \boldsymbol{\varepsilon}>\mathbf{0}$, let $\boldsymbol{\delta}(\boldsymbol{\varepsilon})$ be represented by a sequence $\delta_{n}$. We can suppose that $\delta_{n}>0$ for all $n$. Then there is some $k_{n} \geq N$ with $1 / k_{n}<\delta_{n}$ for every $n$, the sequence $y_{n}^{\prime}=y_{k_{n}}$ represents an element $\mathbf{y}^{\prime} \in M(z)$, and we have $d_{i *}\left(\mathbf{y}^{\prime}\right)<\boldsymbol{\delta}(\boldsymbol{\varepsilon})$. So $d_{j *}\left(h_{*}\left(\mathbf{y}^{\prime}\right)\right)<\boldsymbol{\varepsilon}$, which contradicts 16.2 .

\section{Strongly equicontinuous Foliated SPACES}

Let $(X, \mathcal{F})$ be a compact foliated space. Compact generation and recurrence are properties satisfied by its holonomy pseudogroup with the generators given by a finite defining cocycle. To see this, fix a finite defining cocycle $\left(U_{i}, p_{i}, h_{i, j}\right)$ of $\mathcal{F}$ with $p_{i}: U_{i} \rightarrow Z_{i}$ and $h_{i, j}: Z_{i, j} \rightarrow Z_{j, i}$, where $Z_{i, j}=p_{i}\left(U_{i} \cap U_{j}\right)$. Let $\mathcal{H}$ be the representative of the holonomy pseudogroup of $\mathcal{F}$ induced by $\left(U_{i}, p_{i}, h_{i, j}\right)$ on $Z=\bigsqcup_{i} Z_{i}$. Suppose that $\left(U_{i}, p_{i}, h_{i, j}\right)$ is a shrinking of another defining cocycle $\left(\widetilde{U}_{i}, \tilde{p}_{i}, \tilde{h}_{i, j}\right)$ with $\tilde{p}_{i}: \widetilde{U}_{i} \rightarrow \widetilde{Z}_{i}$ and $\tilde{h}_{i, j}: \widetilde{Z}_{i, j} \rightarrow \widetilde{Z}_{j, i}$, where $\widetilde{Z}_{i, j}=\tilde{p}_{i}\left(\widetilde{U}_{i} \cap \widetilde{U}_{j}\right)$. This means that, for each $i, \bar{U}_{i} \subset \widetilde{U}_{i}, Z_{i}=\tilde{p}_{i}\left(U_{i}\right)$, and $p_{i}$ is the restriction of $\tilde{p}_{i}$. Thus $\overline{Z_{i}} \subset \widetilde{Z}_{i}, \overline{Z_{i, j}} \subset \widetilde{Z}_{i, j}$, and $h_{i, j}$ is the restriction of $\tilde{h}_{i, j}$. Let $\widetilde{\mathcal{H}}$ be the representative of the holonomy pseudogroup of $\mathcal{F}$ induced by $\left(\widetilde{U}_{i}, \tilde{p}_{i}, \tilde{h}_{i, j}\right)$ on $\widetilde{Z}=\bigsqcup_{i} \widetilde{Z}_{i}$. It easily follows that $Z$ is relatively compact in $\widetilde{Z}, \mathcal{H}$ is the restriction of $\widetilde{\mathcal{H}}$ to $Z, Z$ meets all $\widetilde{\mathcal{H}}$-orbits, and the transformations $h_{i, j}$ form a system of compact generation of $\widetilde{\mathcal{H}}$ on $Z$. This system is proved to be recurrent as follows. Fix any point $x$ in the closure of some 
$Z_{i}$ in $\widetilde{Z}_{i}$. Then $\tilde{p}_{i}^{-1}(x)$ cuts some $\widetilde{U}_{j}$. Thus $V_{i, j}=\tilde{p}_{i}\left(\widetilde{U}_{i} \cap U_{j}\right)$ is a neighborhood of $x$ in $\widetilde{Z}_{i}$ such that $V_{i, j} \subset \widetilde{Z}_{i, j}, V_{i, j} \cap Z_{i}=Z_{i, j}$ and $\tilde{h}_{i, j}\left(V_{i, j}\right) \subset Z_{j}$. Hence the transformations $h_{i, j}$ form a recurrent system of compact generation by Lemma 4.4. Therefore, according to Theorem 4.6, the coarse quasi-isometry type of the $\mathcal{H}$-orbits with the metric induced by the generators $h_{i, j}$ is kept uniformly fixed when varying the defining cocycle. So each leaf of $\mathcal{F}$ determines a coarse quasi-isometry type of metric spaces. See e.g. [11] for a more detailed description of the coarse quasi-isometry between the leaves and the orbits of the holonomy pseudogroup, which is already implicit in [22]. The bornotopy type of leaves is studied in [13], and most of its discussion also applies to the coarse quasi-isometry type.

If $\mathcal{F}$ is at least of class $C^{1}$, then there exists a metric tensor on the leaves that is continuous on $X$. In this case, it is well known that the already explained embedding of $Z$ into $X$, as a complete transversal of $\mathcal{F}$, defines a uniform collection of coarse quasi-isometries between the $\mathcal{H}$-orbits and the corresponding leaves. This is just a consequence of having a uniform upper bound of the diameter of the plaques for the given finite defining cocycle. Therefore the coarse quasi-isometry type determined by each leaf is given by just itself with such a metric tensor.

The foliated space $(X, \mathcal{F})$ will be called weakly equicontinuous, strongly equicontinuous, quasi-analytic, or quasi-effective, respectively, if any representative of its holonomy pseudogroup is such. This is well defined because all of these properties on pseudogroups are invariant by equivalences (Lemmas $8.2,8.8$ and 9.5 ). Then Theorem 10.1 has the following consequence.

Theorem 17.1. Let $(X, \mathcal{F})$ be an equicontinuous, compact and quasi-effective foliated space. Assume that the space of leaves is connected (for example, if $\mathcal{F}$ is transitive, or if $X$ is connected). Then all leaves with trivial holonomy group determine the same coarse quasi-isometry type.

When $(X, \mathcal{F})$ is at least of class $C^{2}$, the leaves can be endowed with a metric tensor which is continuous on $X$, and the above result can be improved to obtain quasi-isometries via diffeomorphisms between covers of leaves.

This will be done with the help of the normal quasi-foliated bundles of Section 1 As the previous results make evident, the problem with the holonomy and topological structure of the transversal makes it difficult to effectively use the equicontinuity to push whole leaves onto others. In any case, the following weaker solution to this problem can be provided.

Theorem 17.2. Let $(X, \mathcal{F})$ be a strongly equicontinuous, compact foliated space of class $C^{2}$ with connected space of leaves (for example, if $\mathcal{F}$ is transitive, or if $X$ is connected). Then the universal covers of all the leaves are uniformly quasi-isometric via diffeomorphisms.

Proof. Let $L^{\prime}$ be the universal cover of a leaf $L$, and let $N\left(L^{\prime}\right)$ be the normal bundle described in Section 1. With respect to some metric on $N\left(L^{\prime}\right)$, which is boundedly distorted via the embedding, there is a product neighborhood $N\left(L^{\prime}, \varepsilon_{0}\right)$ of the zero section in $N\left(L^{\prime}\right)$ carrying a lamination $Y$, as described in Theorem 1.3 The projection of the leaves in this neighborhood onto the zero section $L^{\prime}$ is locally a diffeomorphism with bounded distortion, by Proposition 1.5 On the other hand, the strong equicontinuity of the pseudogroup readily implies that these projections are actually covering maps. More precisely, with the notation of Section 1 strong equicontinuity implies that given $\varepsilon>0$ there exists $\delta>0$ such that if $S$ is a leaf of $Y$ and meets the fiber $p^{-1}(x)$ of some point $x \in L^{\prime}$ at a point at distance $<\delta$ from $x$, then it meets every fiber $p^{-1}(y)$ at points at distance $<\varepsilon$ from the base $y$. This implies that the projection $p: S \rightarrow L^{\prime}$ is a covering map, for it is a local homeomorphism which has the path covering property, the only obstruction to lifting a path being that such 
leaf $S$ runs off the neighborhood $N\left(L^{\prime}, \varepsilon_{0}\right)$. Since $L^{\prime}$ is simply connected, it follows that $p$ is a diffeomorphism. That $p$ has bounded distortion was already discussed in Proposition 1.5

The above paragraph shows that universal covers of pairs of leaves are uniformly quasiisometric if both leaves are close enough. Then the result follows since the space of leaves is compact and connected.

Theorem 17.3. Let $(X, \mathcal{F})$ be a strongly equicontinuous, compact and quasi-effective foliated space of class $C^{2}$ with connected space of leaves (for example, if $\mathcal{F}$ is transitive, or if $X$ is connected). Then the holonomy covers of all the leaves are uniformly quasi-isometric via diffeomorphisms.

Proof. The proof is similar to the proof of Theorem 17.2, but using the holonomy cover $L^{\prime \prime}$ of a given leaf $L$ instead of using the universal cover $L^{\prime}$. As $N\left(L^{\prime}, \varepsilon_{0}\right)$ in the above proof, the neighborhood $N\left(L^{\prime \prime}, \varepsilon_{0}\right)$ of the zero section in the normal bundle $p: N\left(L^{\prime \prime}\right) \rightarrow L^{\prime \prime}$, carrying a lamination $Y$, satisfies the following property: given $0<\varepsilon<\varepsilon_{0}$, there exists $\delta>0$ such that, if $S$ is a leaf of $Y$ meeting some fiber $p^{-1}(x)$ of a point $x \in L^{\prime \prime}$ at a point at distance $<\delta$ from $x$, then $S$ meets every fiber $p^{-1}(y)$ at a distance $<\varepsilon$ from the base point $y \in L^{\prime \prime}$, and it follows that $p: S \rightarrow L^{\prime \prime}$ is a covering map, whose triviality has to be proved. This would finish the proof because the distortion of $p: S \rightarrow L^{\prime \prime}$ is uniformly bounded for the leaves $S$ of $Y$, as in the proof of Theorem 17.2

Observe that $Z(x, r)=Y \cap p^{-1}(x) \cap N\left(L^{\prime \prime}, \delta\right)$ is a transversal of $Y$ through $x$ for any $r \leq \varepsilon_{0}$. Then the key property of the above statement can be stated as follows: if $h$ is any holonomy map of $Y$ defined on some neighborhood of $x$ in $Z(x, \delta)$, and whose image is contained in $Z(x, \varepsilon)$, then $h$ can be extended to a holonomy transformation with domain $Z(x, \delta)$ and image contained in $Z(x, \varepsilon)$. Moreover, under the present hypothesis, this extension can be assumed to be unique. The only such holonomy transformation is the identity on $Z(x, \delta)$ because $L^{\prime \prime}$ has trivial holonomy group in $Y$ since it is the holonomy cover of $L$. Therefore any such a leaf $S$ meets every fiber of $p$ at just one point; i.e., $p: S \rightarrow L^{\prime \prime}$ is a diffeomorphism, as desired.

Remark. There are versions of Theorems 17.2 and 17.3 for the coarse quasi-isometry type of the universal coverings or the holonomy covers of all leaves when the foliated space is not of class $C^{2}$; in particular, this generalizes Theorem 17.1. The coarse quasi-isometry types of these covers can be defined again via the generators of a representative of the holonomy pseudogroup induced by a finite defining cocycle: the orbits can be thought as graphs in an obvious way, and thus the corresponding covers can be constructed. The coarse quasi-isometry types of such covers can be proved to be invariant by equivalences when the metrics are induced by recurrent systems of compact generation. Hence, versions of Theorems 4.6 and 10.1 for covers of the orbits need to be proved first. These generalizations are easy to make, but the required notation becomes complicated; thus they are left to the reader.

One of the fundamental results of Molino's theory of Riemannian foliations is the following. The closure of the leaves partition the manifold into the leaves of a larger singular foliation [17]. In the general situation considered here, the following weaker results are available; they follow directly by applying Theorem 11.1, Corollary 11.2 and Corollary 12.4 to the holonomy pseudogroup.

Theorem 17.4. Let $(X, \mathcal{F})$ be a strongly equicontinuous, compact foliated space. Then the closure of each leaf is a minimal set. In particular, $\mathcal{F}$ is minimal if it is transitive. 
Corollary 17.5. The leaf closures define a partition of any strongly equicontinuous, compact foliated space.

Theorem 17.6. Let $(X, \mathcal{F})$ be a strongly equicontinuous, compact and quasi-effective foliated space. Then the closure of each leaf is a homogeneous space

The next result shows another geometric aspect of the structure of a strongly equicontinuous foliated space. It was shown by H. Winkelnkemper [31] for the holonomy groupoid or graph, and by F. Alcalde Cuesta [1] for the homotopy groupoid of a Riemannian foliation.

Corollary 17.7. Let $(X, \mathcal{F})$ be a strongly equicontinuous, compact foliated space of class $C^{2}$ with connected leaf space. Then the homotopy groupoid of $(X, \mathcal{F})$ is, canonically, a fiber bundle whose structural group can be reduced to the group of differentiable quasiisometries of the typical fiber, this being the universal cover of a leaf. If $\mathcal{F}$ is quasi-effective, then the same is true for the holonomy groupoid, the fiber now being the holonomy cover of a leaf.

Proof. The homotopy groupoid $G$ of $(X, \mathcal{F})$ consists of equivalence classes of paths on leaves, two paths $\alpha$ and $\beta$ being equivalent if they have the same endpoints and the closed loop $\alpha \beta^{-1}$ is homotopically trivial in the leaf which contains it. If $[\alpha]$ is a point of $G$, then let $s[\alpha]=\alpha(0)$ denote the source map $s: G \rightarrow X$. The fiber of $s$ over a point $x \in X$ is $G_{x}$, and is canonically identified with the universal cover of the leaf $L_{x}$. The foliated space being of class $C^{1}$ means that its leaves can be endowed with a continuous metric tensor. Let $U$ be a flow box for $X$ with leaf space $Z$ and such that its plaques are convex subsets of the leaves with respect to the chosen metric tensor. Let $x \in U$, and let $P$ be the plaque containing $x$, so that $U$ is of the form $P \times Z$. Then, if $G_{U}$ denotes the restriction of $G$ to $U, G_{U}=s^{-1} U$, there is a map $G_{U} \rightarrow Z \times G_{P}$ which sends a point $[\alpha] \in U$ to $(q(\alpha(0)),[p \varnothing \alpha(0)])$, where $q: U \rightarrow Z$ is the projection into the space of leaves of $U$. Since the plaque $P$ is convex, there is a unique geodesic path in $P$ joining $x$ to any given point of $P$, so there is a well defined map $G_{P} \rightarrow P \times G_{x}$ obtaining by precomposing a path starting at some $y \in P$ with the unique geodesic in $P$ from $x$ to $y$. Let $\phi: G_{U} \rightarrow U \times G_{x}$ denote the composition of this two maps. This map is a homeomorphism, and from the previous work (Theorem 17.2), it follows that this map is a quasi-isometry on the fibers, that is, it sends the fiber $s^{-1}(y)=G_{y}$ quasi-isometrically onto $G_{x}$. The quasi-isometry distortion is bounded, and there is a commutative diagram

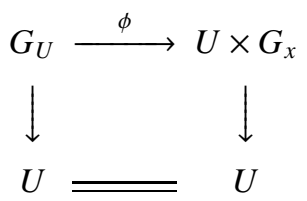

from which the result readily follows.

If the foliated space is quasi-effective, then the same property for the holonomy groupoid is proved similarly by using Theorem 17.3 .

To conclude, it is quite reasonable to expect that the theory presented in this paper can be extended to include larger classes of foliated spaces which have some sort of transverse uniformity, paralleling certain well-known structures of classical topological dynamics [28], e.g., distal actions.

\section{REFERENCES}

[1] F. Alcalde Cuesta, Groupoïde d'homotopie d'un feuilletage riemannien et réalisation symplectique de certaines variétés de Poisson, Publ. Mat. 33 (1989), 395-410. 
[2] J.A. Álvarez López and A. Candel, Topological description of Riemannian foliations with dense leaves, preprint.

[3] J. A. Álvarez López and A. Candel, Generic geometry of leaves, (Forthcomming preprint).

[4] J. Block and S. Weinberger, Aperiodic tilings, positive scalar curvature, and amenability of spaces, J. Amer. Math. Soc. 5 (1992), 907-918.

[5] A. Candel and L. Conlon, Foliations I, Graduate Studies in Mathematics, American Mathematical Society, Providence, 2000.

[6] S.E. Goodman and J.F. Plante, Holonomy and averaging in foliated sets, J. Diff. Geom. 14, 401-407.

[7] M. Gromov, Asymptotic invariants of infinite groups, Geometric Group Theory, Volume 2, G.A. Niblo, M.A. Roller, eds., Cambridge University Press, Cambridge, 1993.

[8] A. Haefliger, Pseudogroups of local isometries, Differential Geometry (Santiago de Compostela 1984), L.A. Cordero, ed., Research Notes in Math. 131, Pitman Advanced Pub. Program, Boston, 1985, pp. 174-197.

[9] A. Haefliger, Leaf closures in Riemannian foliations, A Fête on Topology, Y. Matsumoto, T. Mizutani and S. Morita, eds., Academic Press, New York, 1988, pp. 3-32.

[10] A. Haefliger, Foliations and compactly generated pseudogroups, preprint, 2001.

[11] G. Hector and U. Hirsch, Introduction to the Geometry of Foliations, Parts A and B, Aspects of Mathematics, vols. E1 and E3, Friedr. Vieweg and Sohn, Braunschweig, 1981 and 1983.

[12] M. Hirsch, Differential Topology, Graduate Texts in Mathematics 33, Springer-Verlag, New York, 1976.

[13] S. Hurder, Coarse geometry of foliations, Geometric Study of Foliations (Tokyo 1993), T. Mizutani, K. Masuda, S. Matsumoto, T. Inaba, T. Tsuboi, Y. Mitsumatsu, eds., World scientific Publishing Co. Pte. Ltd., Singapore, 1994, pp. 35-96.

[14] S. Hurder and A. Katok, Ergodic theory and Weil measures for foliations, Annals of Math. 126 (1987), 221-275.

[15] M. Kanai, Rough isometries, and combinatorial approximations of geometries of non-compact manifolds, J. Math. Soc. Japan 37 (1985), 391-413.

[16] M. Kellum, Uniformly quasi-isometric foliations, Ergodic Theory Dynamical Systems 13 (1993), 101-122.

[17] P. Molino, Riemannian Foliations (with appendices by G. Cairns, Y. Carrière, E. Ghys, E. Salem and V. Sergiescu), Progress in Mathematics, vol. 73, Birkhäuser, Boston, 1988.

[18] C.C. Moore and C. Schochet, Global Analysis on Foliated Spaces, MSRI Publications, vol. 9, SpringerVerlag, New York, 1988.

[19] J.R. Munkres, Topology: a First Course, Prentice-Hall, Inc., Englewood Cliffs, N.J., 1975.

[20] J. Nagata, Modern General Topology, (Second, revised edition), Noth-Holland Publishing Company, Amsterdam, 1974.

[21] A. Robinson, Non-standard Analysis, (Reprint of the 1974 Edition), Princeton University Press, Princeton, N.J., 1996.

[22] J.F. Plante, Foliations with measure preserving holonomy, Ann. of Math. 102 (1975), 327-361.

[23] J. Roe, Coarse Cohomology and Index Theory on Complete Riemannian Manifolds, Memoirs of the Amer. Math. Soc., Number 497, 1993.

[24] R. Sacksteder, Foliations and pseudogroups, American J. of Math. 87 (1965), 79-102.

[25] Y.M. Smirnov, On metrization of topological spaces, Amer. Math. Soc. Translations. Series One 8 (1953), $62-77$.

[26] L.A. Steen and J.A. Seebach, Jr., Counterexamples in Topology, (Second Edition), Springer-Verlag, New York-Heidelberg, 1978.

[27] C. Tarquini, Feuilletages conformes, Ann. Inst. Fourier 54 (2004), 453-480.

[28] W. Veech, Topological dynamics, Bull. Amer. Math. Soc. 83 (1977), 775-830.

[29] A. Weil, L'Integration dans les Groupes Topologiques et ses Applications (Second Edition), Actualités Scientifiques et Industrielles, no. 1145. Publications de l'Institut de Mathematique de l'Universite de Strasbourg 4, Hermann, Paris, 1951.

[30] S. Willard, General Topology, Addison-Wesley Publishing Co., Reading, Mass. 1970.

[31] H. E. Winkelnkemper, The graph of a foliation, Ann. Global Anal. Geom. 1 (1983), 51-75.

* Departamento de Xeometría e Topoloxía, Facultade de Matemáticas, Universidade de Santiago de Compostela, Campus Universitario Sur, 15706 Santiago de Compostela, Spain

E-mail address: jesus.alvarez@usc.es

$\dagger$ Department of Mathematics, CSUN, Northridge, CA 91330, U.S.A.

E-mail address: alberto.candel@csun.edu 